University of Louisville

ThinkIR: The University of Louisville's Institutional Repository

Electronic Theses and Dissertations

8-2011

\title{
Consistency of differentially expressed gene rankings based on subsets of microarray data.
}

Dake Yang

University of Louisville

Follow this and additional works at: https://ir.library.louisville.edu/etd

\section{Recommended Citation}

Yang, Dake, "Consistency of differentially expressed gene rankings based on subsets of microarray data." (2011). Electronic Theses and Dissertations. Paper 1615.

https://doi.org/10.18297/etd/1615

This Master's Thesis is brought to you for free and open access by ThinkIR: The University of Louisville's Institutional Repository. It has been accepted for inclusion in Electronic Theses and Dissertations by an authorized administrator of ThinkIR: The University of Louisville's Institutional Repository. This title appears here courtesy of the author, who has retained all other copyrights. For more information, please contact thinkir@louisville.edu. 


\title{
CONSISTENCY OF DIFFERENTIALLY EXPRESSED GENE RANKINGS BASED ON SUBSETS OF MICROARRAY DATA
}

By

Dake Yang

\begin{abstract}
A Thesis
Submitted to the Department of Bioinformatics and Biostatistics University of Louisville

In Fulfillment of the Requirements

for the Degree of
\end{abstract}

Master of Science

Department of Bioinformatics and Biostatistics

University of Louisville

Louisville, KY

August 2011 
Copyright 2011 by Dake Yang

All rights reserved 



\title{
Consistency of Differentially Expressed Gene Rankings Based on Subsets of Microarray Data
}

\author{
By
}

Dake Yang

A Thesis Approved on

by the following Thesis Committee:

Thesis Director (Guy Brock)

Rudolph S. Parrish 


\section{ABSTRACT \\ CONSISTENCY OF DIFFERENTIALLY EXPRESSED GENE RANKINGS BASED ON SUBSETS OF MICROARRAY DATA \\ Dake Yang}

\section{July 21, 2011}

Data derived from gene expression microarrays are frequently used to identify candidate genes which can characterize and distinguish between two biological phenotypes. A key step in this process is the selection of an appropriate test statistic to identify which genes are differentially expressed between the two tissues. Although many methods have been explicitly developed for this purpose, the traditional $t$-test still remains a popular choice. In this study, we evaluate the empirical impact of choice of test-statistic on the resulting list of differentially expressed genes, in particular when the available sample size is small.

We evaluated several different methods for detecting differentially expressed genes ( $t$-test, empirical Bayes, and SAM) using ten different publicly available data sets. First, we obtained gene lists based on the full data using the different methods. Then, we selected subsamples from the full data, and obtained gene lists based on these subsamples. The consistency was quantified using several scores. Factors evaluated in the empirical study included the size of the subset and the length of the differentially expressed gene list.

We found that when the sample size of the subset is small, the resulting gene list based on the t-test has a very low consistency, while empirical Bayes and SAM have much higher consistencies. This result is particularly evident when considering only the top ranked genes. When sample sizes are larger, all three methods have the 
same performance. We recommend that investigators use these moderated versions in lieu of the t-test when the sample size is small. 


\section{TABLE OF CONTENTS}

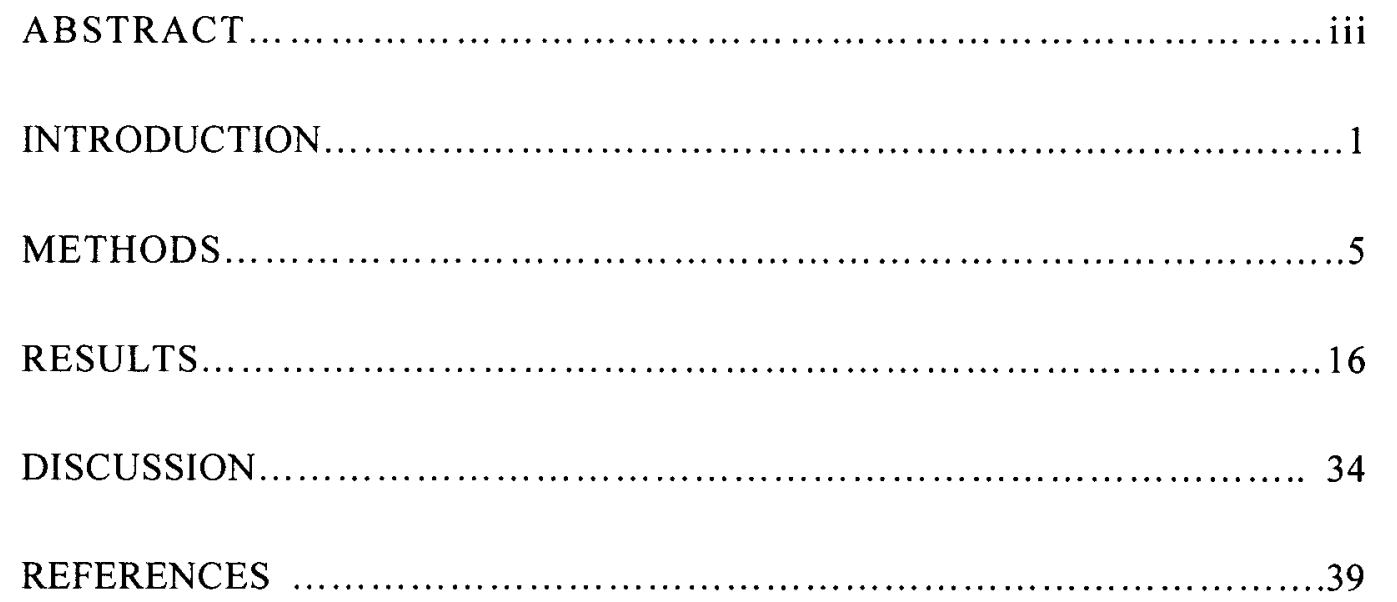

APPENDIX A......................................................... 42

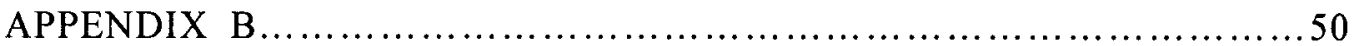

CURRICULUM VITAE.................................................. 76 


\section{CHAPTER I}

\section{INTRODUCTION}

DNA microarray is a tool in biotechnology for comparing the expression of genes on a genomic scale across two or more samples of messenger RNA (mRNA). The microarray consists of thousands of features, which are small DNA oligonucleotides. Each feature contains a particular gene sequence, called a probe, which allows the microarray to simultaneously monitor the relative expression of mRNAs in two or more tissue samples for thousands of genes within the cell. In microarray experiments, one common but important question is how to identify those genes whose expression level changes across different experimental samples or different types of tissue samples (Parrish 2009). Thus, the goal of differential gene expression analysis is to determine the genes whose expression level changes significantly according to the experimental condition.

The microarray is a novel tool for comparing the expression of thousands of genes, but it also brings to light new statistical problems because of the high dimensionality of the data, with a relatively small number of replicates. There are two main issues with microarray data (Xu 2009). First, microarray gene expression data are commonly considered to be highly noisy, because there are many flaws inherent in the current technology. Noise comes from two primary sources in microarray experiments, biological noise and technical noise (Tu 2002). Biological noise consists 
of the inherent variation from patient to patient, the variation in the tissues sampled, and the variation in the cellular composition of the tissue that is subsequently hybridized to the array. Technical noise comes from the differences in sample processing and experimental variables. Secondly, microarray data always contain a much larger number of genes (usually several thousand or more features) relative to the number of samples (usually less than one hundred). With the large number of hypothesis tests being conducted and a relatively small sample size, many spurious results can appear and many biologically meaningful genes can fail to reach statistical significance. In gene expression analysis, a gene is considered differentially expressed when its expression level changes systematically between two treatment conditions (McCarthy and Smyth 2009). The biological significance is typically measured by the relative expression level between the two conditions (the fold-change), while the statistical significance is measured by comparison of a test-statistic to a reference distribution (the p-value). Ranking of significant genes on the basis of the two methods does not always agree, leading investigators to often make comprises between the two approaches.

Due to the huge data volume and inherent variation in microarrays, several statistical methods have been proposed to address these problems (Baldi 2001; Efron 2009). In our paper, we discuss three commonly used methods for identifying which genes are differentially expressed between two different experimental conditions or tissue types. They are the $t$-test, the significance analysis of microarrays (SAM) (Tusher 2001), and the empirical Bayesian (Smyth 2005) methods. In each case, the hypotheses been tested for each gene are the null hypothesis $\mathrm{H}_{0}$ : the gene is not differentially expressed between two different experimental conditions or tissue types, and the alternative hypothesis $\mathrm{H}_{\mathrm{A}}$ : the gene is differentially expressed between two 
different experimental conditions or tissue types. The $t$-test is a traditional method for detecting differentially expressed genes in microarray analysis. The $t$ statistic can be used to determine which genes are significantly differentially expressed by comparing the test statistic to a t-distribution with $n-2$ degrees of freedom, where $n$ is the total number of microarrays. But in the $t$-test method, a gene with a low expression level but small variance can result in a large absolute $t$ statistic even for small mean differences between the two conditions. So this gene can be declared to be differentially expressed, even if the difference in expression is not biologically meaningful.

To solve this problem, alternative methods have been proposed. To solve the small variance problem of the $t$-test, SAM modifies the standard $t$-statistic by adding a small 'fudge factor' to the variance in the denominator. The SAM method compares this modified test statistic to an expected value under the null hypothesis, which is determined by permutations at the gene expression measurements. Differences between observed and expected values which are above a threshold are considered statistically significant, where the threshold is determined by the desired false discovery rate. Smyth et al. 2004 proposed a similarly derived empirical Bayes approach, which shrinks the estimated sample variances towards a pooled estimate. Both SAM and empirical Bayes estimators are designed to have robust performance, particularly for experiments with small numbers of arrays.

Although these statistical methods have been proposed as improved methods to determine differential gene expression, not too many studies (Haslett 2002; Jeffery 2006; Hong and Breitling 2008) have empirically compared the performances of these different statistical methods. Thus, it is important to evaluate the success of these methods through empirical comparisons. The traditional $t$-test still remains a popular 
choice among biologists, despite the reputed disadvantages in this method when the available sample size is small. So, the goal of this thesis research is to compare and evaluate how well the three methods (the $t$-test, SAM, and empirical Bayes) perform with real microarray data. To accomplish this, we evaluate the consistency in determining differentially expressed genes for each method, based on numerical measures of the degree of overlap between two gene lists (Zhang 2009). Further, we evaluate the consistency for each method, when comparing differentially expressed gene lists obtained from subsets of two different datasets of the same disease. We consider that the methods are performing well in detecting the differentially expressed gene lists when the scores are high. We define that one method is better than the other, when the scores calculated by one method are higher than other scores from the other method under the same situation.

The rest of this thesis is organized as follows. In Chapter 2, section 2.1 we describe the three DEGs (differentially expressed gene) detecting methods (the $t$-test, SAM, and empirical Bayes). In section 2.2, we describe the scores which are used to measure the consistency between two gene lists (POG, POGR, nPOG and nPOGR). We describe the two groups of data sets for evaluating the consistency of DEdetection methods in sections 2.3.1 and 2.3.2. Chapter 3 gives the results from our study, and Chapter 4 is the discussion 


\section{CHAPTER II}

\section{METHODS}

\subsection{Methods for detecting the differentially expressed genes}

We selected three popular methods ( $t$-test, empirical Bayes and significance analysis of microarrays (SAM)) for determining differentially expressed genes (DEGs) in microarray data. The standard notations for the three methods we defined as follows. Suppose the goal is to detect differentially expressed genes between two classes of samples, normal and diseased. Suppose group one (normal) contains $n$ samples and the group two (diseased) contains m samples. Suppose there are gene expression measurements for all the $G$ genes. For the any given $i$ th gene $(\mathrm{i}=1,2, \ldots G)$, let $\bar{X}_{1 i}$ be the mean of the $i$ th gene expression measurements amongst the samples from group one, with corresponding standard deviation $S_{X_{1 i}}$. Similarly, define $\bar{X}_{2 i}$ and $S_{X_{2 l}}$ to be the mean and standard deviation of the $i$ th gene expression measurements from the samples of group 2. The hypothesis being tested for each of genes is $\mathrm{H}_{0}: \mu_{1 i}=\mu_{2 i}$ vs $\mathrm{H}_{\mathrm{A}}: \mu_{1 i} \neq \mu_{2 i}$, where $\mu_{1 i}$ and $\mu_{2 i}$ are the population mean expression measurements for the gene in the two groups. 


\subsubsection{T-test}

The two-sample $t$-test on each gene can be used to detect which genes are differentially expressed between two classes of samples. The $t$-statistic for the $i$ th gene assuming using a pooled estimator for the standard deviation is defined as follows:

$$
t_{i}=\frac{\left(\bar{X}_{1 i}-\bar{X}_{2 i}\right)}{\sqrt{\frac{n S_{X_{1 i}}^{2}+m S_{X_{21}}^{2}}{n+m-2} \sqrt{\frac{1}{n}+\frac{1}{m}}}}
$$

The gene-specific $t$-test uses only the gene expression values specific to each gene to calculate differential expression. Under the null hypothesis, the $t$-statistic follows a $t$-distribution with approximately $n+m-2$ degrees of freedom. The $P$-value for the two-sided hypothesis test is calculated as $2 P\left(T_{n+m-2} \geq t_{i}\right)$, where $T_{n+m-2}$ denotes a random variable with a $t$ distribution with $n+m-2$ degrees of freedom. The null hypothesis is rejected if the $P$-value is less than the $\alpha$ level of the test.

\subsubsection{SAM (Significance Analysis of Microarrays)}

With the high levels of noise in microarray data and the small sample sizes, the adequacy of the $t$-statistic is questionable. Since there are a large number of genes inmicroarray datasets, there will always be some genes which have a low standard deviation by chance. Therefore, these genes will have a large t-statistic and will be falsely predicted to be differentially expression genes. Several authors have proposed improvements on the $t$ test for genome-wide expression measurements.

To improve upon the deficiencies of the $t$-test mentioned above, Tusher (Tusher 2001) proposed the significance analysis of microarrays (SAM) method to 
select differentially expressed genes by adding a small, strictly positive constant $S_{0}$, (the so called fudge factor) to the denominator of the usual $t$-statistic. Define gene specific "relative differences" $d_{i}(i=1,2, \ldots G)$ and gene specific standard deviations $S_{i}(i=1,2, \ldots G)$.

$$
\begin{gathered}
d_{i}=\frac{\bar{X}_{1 i}-\bar{X}_{2 i}}{S_{i}+S_{0}}, \\
S_{i}=\sqrt{\frac{1 / n+1 / m}{n+m-2}\left\{\sum_{j=1}^{n}\left(X_{1 i j}-\bar{X}_{1 i}\right)^{2}+\sum_{j=1}^{m}\left(X_{2 i j}-\bar{X}_{2 i}\right)^{2}\right\}} .
\end{gathered}
$$

The fudge factor $S_{0}$ is calculated by minimizing the coefficient of variation of $d_{i}$ as a function of $S_{i}$ (Tusher 2001). A permutation procedure is used to calculate the $P$-value for each gene.

DE genes are determined by comparing the observed relative differences $d_{i}$ with the expected relative differences $d_{i}^{E}$, where $d_{i}^{E}$ are calculated by averaging the relative differences from permutations of the data. Genes with differences between observed and expected values greater than a threshold $\Delta$ are declared statistically significant. The threshold $\Delta$ is a user controlled parameter, and SAM provides an estimate of the FDR (false discovery rate) for each $\Delta$ value.

\subsubsection{Empirical Bayes}

A hierarchical model (Smyth 2004; Smyth 2005) is used to model distribution of the variances and differences in mean expression between the two tissue types for each gene. Based on the hierarchical model for the variances, for each gene, a posterior estimate of the population variance $\sigma_{i}^{2}$ for each gene, given the sample 
variance $S_{i}^{2}$, is obtained. This expectation is termed $\$_{i}^{2}$, and is calculated as a weighted average of the prior variance $S_{0}^{2}$ and the sample variance $S_{i}^{2}$,

$$
\oiint_{i}^{2}=\frac{d_{0} S_{0}^{2}+d_{i} S_{i}^{2}}{d_{0}+d_{i}}
$$

Where $d_{0}$ is the degrees of freedom for the prior distribution of $S_{i}^{2}$ and $d_{i}$ is the degrees of freedom for the ordinary $t$-statistic. The moderated $t$-statistic is then defined as

$$
\tilde{t}_{i}=\frac{\bar{X}_{l i}-\bar{X}_{2 i}}{S_{i} \sqrt{\frac{1}{n+m}}}
$$

The moderated $t$-statistic follows a $t$-distribution with $d_{i}+d_{0}$ degrees of freedom, under the null hypothesis.

\subsection{POG and POGR scores}

In this section, we define the metrics used to measure the consistency between two gene lists. For example, two separate lists of differentially expressed genes produced by independent studies of the same disease. A relatively straightforward metric is the percentage of overlapping genes (POG) metric, which has been used by numerous previous studies (Shi 2006; Chen 2007). The POG measures the consistency of two separate gene lists by determining the percentage of overlapped genes between the two lists. Specifically, assume that $n$ genes are shared between list 1 with length $l_{1}$, and list 2 with length $l_{2}$. The calculation of the POG score depends on which list is determined to be the 'reference' list, i.e. the POG between list 1 and list 2 is $P O G_{12}=\frac{n}{l_{1}}$ and the POG between list 2 and list 1 is $P O G_{21}=\frac{n}{l_{2}}$. The POG is 
a useful metric to evaluate the reproducibility of gene lists from independent studies, and has been previously used to measure the consistency of gene lists obtained from separate studies of the same disease (Zhang 2009). We can also consider the POG score as equivalent to a popular index of diagnostic accuracy, the sensitivity.

To see this, assume that the reference list (say, list 1) contains the 'true' differentially expressed genes, equivalent to the individuals which truly have the disease of interest in the evaluate of a diagnostics test. Then, the length of list $1\left(l_{1}\right)$ is the same as the denominator of the sensitivity, and the length of the overlap of the two lists $(n)$ is the same as the numerator of the sensitivity.

An extension to the POG score, called the percentage of overlapping genes-related or POGR score, considers not only those genes which are shared between the two lists, but also those genes which are highly correlated with each other. The motivation here is to consider a measure of the reproducibility of gene lists, which accounts for the coordinated molecular changes which frequently occur in biological systems. Similar to the POG score, the POGR (percentage of overlapping genes-related) metric is used to evaluate the consistency between two gene lists. Let $n_{r 12}$ (or $n_{r 21}$ ) represent the number of genes between two lists which are not shared but are significantly correlated. Here, two genes are defined as a correlated pair if and only if they are significantly correlated in both datasets. Then, the formula for the POGR scores are,

$$
P O G R_{12}=\left(n+n_{\mathrm{r} 12}\right) / l_{1} \text { or } P O G R_{21}=\left(n+n_{\mathrm{r} 21}\right) / l_{2} \text {. }
$$

Both the POG and POGR are in the range of 0 to 1 . Generally, a high POG or 
POGR score is wanted between two lists. A problem with both, however, is that they are positively correlated with the length of the lists. Further, when the expressed genes are highly correlated with each other in a biological system, the POGR score between two randomly selected gene lists can be high, since $n_{r 12}$ (or $n_{r 21}$ ) may be high. This is because that, when the genes are highly correlated, the $n_{\mathrm{r} 12}$ maybe high. To solve this problem, Zhang et al, (2009) introduced normalized versions of the POG and POGR scores (nPOG, nPOGR), where described below.

Define $E(n)$ as the expected number of the shared genes between two randomly generated lists with lengths $l_{1}$ and $l_{2}$ lists. Then, the expected POG is

$$
E\left(P O G_{12}\right)=E(n) / l_{1} \text { or } E\left(P O G_{21}\right)=E(n) / l_{2}
$$

The normalized POG is similar in calculation to the kappa coefficient, which represents a chance-corrected index of agreement between categorical variables. The $\mathrm{E}\left(P O G_{12}\right)$ [or $\left.\mathrm{E}\left(P O G_{21}\right)\right]$ can be estimated by simulation, by averaging the POG scores from 10,000 pairs of randomly generated lists (with lengths $l_{1}$ and $l_{2}$ ). Randomly selected lists of length $l_{1}$ (or $l_{2}$ ) can be generated by drawing $l_{1}$ (or $l_{2}$ ) genes without replacement from the complete list of genes. The POG as the ratio of the observed scores to the maximum potential scores as follows:

$$
\begin{aligned}
& n P O G_{12}=\frac{P O G_{12}-E\left(P O G_{12}\right)}{1-E\left(P O G_{12}\right)}=\frac{n-E(n)}{l_{1}-E(n)} \\
& n P O G_{21}=\frac{P O G_{21}-E\left(P O G_{21}\right)}{1-E\left(P O G_{21}\right)}=\frac{n-E(\mathrm{n})}{l_{2}-E(n)}
\end{aligned}
$$

We can normalize the POGR score in a similar way. The $E\left(P O G R_{12}\right)$ or 
[E(POGR $\left.\left.R_{21}\right)\right]$ can be calculated by adding the expected number of correlated genes between the two lists, $E\left(n_{r 12}\right)$ [or $E\left(n_{r 21}\right)$ ], to the expected number of overlapped genes, $E(n)$. So, the normalized POGR score is

$$
\begin{gathered}
n P O G_{12}=\frac{P O G R_{12}-E\left(P O G R_{12}\right)}{1-E\left(P O G R_{12}\right)}=\frac{n+n_{r 12}-E(n)-E\left(n_{r 12}\right)}{l_{1}-E(n)-E\left(n_{r 12}\right)} \\
\text { or } \\
n P O G_{21}=\frac{P O G R_{21}-E\left(P O G R_{21}\right)}{1-E\left(P O G R_{21}\right)}=\frac{n+n_{r 21}-E(n)-E\left(n_{r 21}\right)}{l_{2}-E(n)-E\left(n_{r 21}\right)} .
\end{gathered}
$$

The nPOGR score corrects for chance agreement, and removes the effects of the length of the gene lists and data correlations. Hence, the score may be more appropriate for comparing the consistency levels between differentially expressed gene lists with different lengths and from different studies.

\subsection{Study Design}

\subsubsection{Consistency of DE (differentially expressed) detection methods within a} single dataset.

Four datasets of different diseases were used to evaluate the consistency of methods to detect differential expression, based on subsets of the same data (Table 1). Before all the procedures we filtered the raw data to remove invariant transcripts, using the 'nsFilter' command in the 'genefilter' $\mathrm{R}$ package.

Transcripts were filtered using the 'var.cutoff' with the value set to 0.5 , so that all genes with a variance below 0.5 were removed. This is motivated by the observation that in many tissues only $40 \%$ of the genes are expressed. We additionally set the 


\begin{tabular}{|c|c|c|c|c|c|}
\hline Data set & Reference & $\begin{array}{c}\text { Raw Size } \\
\text { Samples } \times \text { Genes }\end{array}$ & Samples $\times$ Genes & Analysis(number) \\
\hline ALL & Sabina et al., 2004 & $128 \times 12625$ & $79 \times 4399$ & BCR/ABL(37), NEG(42) & Affymetrix \\
ColonCA & Alon et al, 1999 & $62 \times 2000$ & $62 \times 2000$ & Tumor(40), Normal(22) & Affymetrix \\
lungExpression & Beer et al., 2002 & $86 \times 3171$ & $86 \times 3171$ & death(24), live(62) & Affymetrix \\
golubEsets & Golub et al, 1999 & $72 \times 7129$ & $72 \times 2698$ & ALL(47), AML(25) & Affymetrix \\
\hline
\end{tabular}

Table 1. Details of four data sets used to study consistency of DE detection methods within a single data set.

argument 'remove.dupEntrez=TRUE', so that for transcripts mapping to the same Entrez Gene ID, the transcript with the largest variance will be retained and the others removed.

The ALL (Acute Lymphoblastic Leukemia) data from the Ritz Laboratory(Sabina 2004), consists of 128 samples. We selected 79 samples representing two molecular biology subtypes, $\mathrm{BCR} / \mathrm{ABL}$ (an oncogene fusion protein consisting of $\mathrm{BCR}$ and $\mathrm{ABL}$ genes) (37 samples) and NEG (42 samples). After screening out invariant expression profiles, the final number of probes in the data set was 4,399. The ColonCA data contains 2,000 expression measurements from 40 colon cancer tumor samples and 22 normal samples (Alon 1999). The lungExpression data (Beer 2002) consists of 3,171 expression measurements from 86 lung cancer patients, of whom 62 were alive at the end of the study and 24 had died. The golubEsets data (Golub 1999)are the combined training samples and test samples. There are 47 patients with acute lymphoblastic leukemia (ALL) and 25 patients with acute myeloid leukemia (AML). The samples were assayed using Affymetrix Hgu6800 chips and data on the expression of 7129 genes (Affymetrix probes) are available. After screening out invariant probes, a total of 2,698 probes remained. All data sets were stored as normalized gene expression format (class exprSet) in 
Bioconductor.

To evaluate the consistency of the three DE detection methods, we randomly selected a varying number of subsets without replacement from the full data (Four different numbers of subsets were used for each data set). Here we ignored the possible dependency between samples from the same group (diseased / normal) in the design, implicitly assuming that the samples from within a single group were independent. Next, we used three methods to detect the differentially expressed genes lists based on both the subsets and the full data. The genes were then ranked on the basis of the absolute values of their test statistics. Next, we used different lengths of lists to calculate the scores we defined in section 2.2 (POG, nPOG, POGR and nPOGR). We repeated the three steps above 100 times, and calculate the mean of each of scores, for each combination of method, dataset, subset length, and list length.

\subsubsection{Consistency of DE detection methods between data sets}

Six datasets for three diseases were analyzed to evaluate the consistency of subsets from different data sets that were collected for the same disease. The prostate cancer cDNA microarray data consists of 62 tumors and 41 normal prostate samples measured for 46,205 clones (Lapointe 2004). After screening out invariant expression profiles, the final number of probes in the data set was 20,540 . While the oligo microarray (Affymetrix U95Av2) data contain 52 tumor and 50 non-tumor samples(Singh 2002). After screening out invariant probes, a total of 4,399 probes remained. For lung cancer, the cDNA microarray data consist of 13 squamous cell lung cancer and five normal lung specimens(Garber 2001), and 13,822 probes remained after screening. While the data by Affymetrix human U95A oligonucleotide

\begin{tabular}{|l|l|l|l|l|l|}
\hline Data set & Reference & Raw Size & Used Size & Analysiz(number) & Platform \\
\hline
\end{tabular}




\begin{tabular}{|c|c|c|c|c|c|}
\hline & & Samples $\times$ Genes & Samples $\times$ Genes & & \\
\hline prostate cancer & Lapointe et al., 2004 & $103 \times 46,205$ & $103 \times 20540$ & Tumor(62), Normal(41) & cDNA \\
\hline prostate cancer & Singh et al., 2002 & $102 \times 12600$ & $102 \times 4399$ & Tumor(52), Normal(50) & Affymetrix \\
\hline lung cancer & Garber et al., 2001 & $123 \times 24000$ & $123 \times 13822$ & Tumor(67), Normal(56) & cDNA \\
\hline lung cancer & Bhattacharjee et al., 2001 & $203 \times 12600$ & $203 \times 4399$ & Tumor(186), Normal(17) & Affymetrix \\
\hline Duchenne muscular dystrophy & Haslett et al., 2002 & $23 \times 12625$ & $23 \times 4399$ & $\operatorname{DMD}(12)$, Normal(11) & Affymetrix \\
\hline Duchenne muscular dystrophy & Pescatori et al., 2007 & $37 \times 22283$ & $37 \times 6352$ & $\operatorname{DMD}(23)$, Control(14) & Affymetrix \\
\hline
\end{tabular}

Table 2. Details of six data sets used to study consistency of DE detectionmethods

between different datasets for one disease.

arrays consist of 21 squamous cell lung carcinomas and 17 normal lung specimens(Bhattacharjee 2001). After screening, the final number of probes in the data was 4,399 . The two datasets for Duchenne muscular dystrophy (DMD) are based on Affymetrix (Santa Clara, CA) HG-U95Av2 and HG-U133A GeneChips, respectively. One dataset contains 24 samples from 12 DMD patients and 12 unaffected controls(Haslett 2002), and the other consists of 36 samples from 22 DMD patients and 14 controls (Pescatori 2007). After screening, there were 4,399 and 6,352 probes remained for the two data sets. For each disease, we only analyzed the genes that were present on both platforms. The cDNA data were log base 2 transformed and then normalized as median 0 and standard deviation 1 per array. The Affymetrix GeneChip data were normalized by RMA (Robust Multi-array Analysis).

We again used three methods ( $t$-test, empirical Bayes and SAM) to detect the the differentially expressed gene lists from each dataset. To begin, we measured overlap genes from the two data sets of one disease. And the overlap genes are the interesting genes which we detected differentially expressed genes from. Second, we randomly selected a varying number of subsets without replacement from the two data sets for one disease (Four different numbers of subsets were used to each data set). We assumed that the samples in the same group were independent. Next, we used 
three methods ( $t$-test, empirical Bayes and SAM) to detect the differentially expressed gene lists from the two subsets of the two datasets. Genes were subsequently ranked on the basis of the absolute values of their test statistics. And then, we used different lengths of lists to calculate the scores we defined in section2.2 (POG, nPOG, POGR and nPOGR). We repeated the three steps above 100 times, and calculate the mean of each of scores, for each combination of method, dataset, subset length, and list length. We experimented with different cutoffs to determine whether a gene pair was significantly correlated. 


\title{
CHAPTER III
}

\author{
RESULT
}

\subsection{Consistency of DE detection methods using subsets from the same data set}

For the four datasets from the Biocuductor website, we compared the differentially expressed gene lists determined using subsets from the same dataset to test the consistency of the three methods. For the ALL dataset, we obtained various lengths of differentially expressed gene lists using the three methods ( $t$-test, empirical Bayes and SAM) for different sample sizes. We separately chose subsets of size 3,6 , 10 and 25 randomly and without replacement each from 37 BCR/ABL and 42 NEG samples (total sample size of 6, 12,20 and 50 samples, respectively). Test statistics for differential gene expression between the two tissue types for each gene were calculated using each of the three methods. Then, different lengths of gene lists, 50, 100,500 and 1000 , were used to test the overlap of genes with the gene lists taken from the full data. 


\begin{tabular}{|c|c|cccc|}
\hline \multicolumn{7}{|c|}{ POG score for ALL } \\
\hline Method & length/subset & $\mathbf{5 0}$ & $\mathbf{1 0 0}$ & $\mathbf{5 0 0}$ & $\mathbf{1 0 0 0}$ \\
\hline T-test & $\mathbf{6}$ & 0.070 & 0.088 & 0.195 & 0.299 \\
& $\mathbf{1 0}$ & 0.098 & 0.138 & 0.260 & 0.347 \\
& $\mathbf{2 0}$ & 0.266 & 0.327 & 0.421 & 0.475 \\
& $\mathbf{5 0}$ & 0.578 & 0.625 & 0.677 & 0.696 \\
\hline Empirical Bayes & $\mathbf{6}$ & 0.132 & 0.168 & 0.240 & 0.331 \\
& $\mathbf{1 0}$ & 0.152 & 0.192 & 0.289 & 0.365 \\
& $\mathbf{2 0}$ & 0.306 & 0.368 & 0.439 & 0.485 \\
& $\mathbf{5 0}$ & 0.582 & 0.642 & 0.682 & 0.700 \\
\hline SAM & $\mathbf{6}$ & 0.166 & 0.187 & 0.256 & 0.343 \\
& $\mathbf{1 0}$ & 0.167 & 0.205 & 0.298 & 0.373 \\
& $\mathbf{2 0}$ & 0.353 & 0.397 & 0.455 & 0.492 \\
& $\mathbf{5 0}$ & 0.619 & 0.695 & 0.695 & 0.711 \\
\hline
\end{tabular}

Table 3 POG score for ALL data set

The POG scores for the ALL data are in Table 3. From Table 3 we found that the POG scores were low when the length of the lists and the number of the subsets were small. The POG scores increased with larger subsets or longer length of the gene lists. Then, we discovered from the data that, the values under the $t$-test were generally smaller than the values from other two methods. It was more distinct when the length of the lists and the number of the subsets were small. And the values converged to each other when the length of the lists and the number of the subsets went up. 


\begin{tabular}{|c|c|cccc|}
\hline \multicolumn{7}{|c|}{ POGR score for ALL } \\
\hline Method & length/subset & $\mathbf{5 0}$ & $\mathbf{1 0 0}$ & $\mathbf{5 0 0}$ & $\mathbf{1 0 0 0}$ \\
\hline T-test & $\mathbf{6}$ & 0.463 & 0.570 & 0.821 & 0.908 \\
& $\mathbf{1 0}$ & 0.542 & 0.642 & 0.848 & 0.923 \\
& $\mathbf{2 0}$ & 0.826 & 0.852 & 0.905 & 0.946 \\
& $\mathbf{5 0}$ & 0.930 & 0.937 & 0.956 & 0.970 \\
\hline Empirical Bayes & $\mathbf{6}$ & 0.665 & 0.725 & 0.845 & 0.919 \\
& $\mathbf{1 0}$ & 0.681 & 0.740 & 0.864 & 0.926 \\
& $\mathbf{2 0}$ & 0.878 & 0.874 & 0.909 & 0.948 \\
& $\mathbf{5 0}$ & 0.946 & 0.946 & 0.954 & 0.969 \\
\hline SAM & $\mathbf{6}$ & 0.690 & 0.740 & 0.859 & 0.928 \\
& $\mathbf{1 0}$ & 0.699 & 0.747 & 0.869 & 0.931 \\
& $\mathbf{2 0}$ & 0.895 & 0.882 & 0.917 & 0.954 \\
& $\mathbf{5 0}$ & 0.963 & 0.947 & 0.957 & 0.975 \\
\hline
\end{tabular}

Table 4. POGR scores for ALL data

The POGR scores for the ALL data are showed in Table 4. The result of POGR score was quite similar to the POG score. The POGR scores are uniformly higher than the POG scores, reflecting the strong correlation between the genes in the data set. However, the effect of subset size, length of list, and choice of DE detection method were all similar to the POG score results. 


\begin{tabular}{|c|c|llll|}
\hline \multicolumn{7}{|c|}{ nPOG score for ALL } \\
\hline Method & length/subset & $\mathbf{5 0}$ & $\mathbf{1 0 0}$ & $\mathbf{5 0 0}$ & $\mathbf{1 0 0 0}$ \\
\hline T-test & $\mathbf{6}$ & 0.057 & 0.066 & 0.094 & 0.093 \\
& $\mathbf{1 0}$ & 0.086 & 0.117 & 0.164 & 0.154 \\
& $\mathbf{2 0}$ & 0.258 & 0.313 & 0.347 & 0.319 \\
& $\mathbf{5 0}$ & 0.573 & 0.616 & 0.636 & 0.607 \\
\hline Empirical Bayes & $\mathbf{6}$ & 0.120 & 0.148 & 0.144 & 0.135 \\
& $\mathbf{1 0}$ & 0.141 & 0.172 & 0.197 & 0.178 \\
& $\mathbf{2 0}$ & 0.298 & 0.355 & 0.368 & 0.332 \\
& $\mathbf{5 0}$ & 0.576 & 0.633 & 0.642 & 0.613 \\
\hline SAM & $\mathbf{6}$ & 0.155 & 0.166 & 0.162 & 0.151 \\
& $\mathbf{1 0}$ & 0.156 & 0.185 & 0.207 & 0.189 \\
& $\mathbf{2 0}$ & 0.345 & 0.385 & 0.386 & 0.342 \\
& $\mathbf{5 0}$ & 0.614 & 0.687 & 0.656 & 0.626 \\
\hline
\end{tabular}

Table 5. nPOG scores for ALL data

The nPOG scores for the ALL data are showed in Table 5. From Table 5, we found that the nPOG scores were low when the number of the subsets was small. And the nPOG scores went up when the subsets became larger. But, the nPOG score was not affected by the length of the gene list. Also, we found that, the values under the $t$-test were smaller than the values from the other two methods. It was more distinct when the number of the subsets was small. And the values approached each other when the subsets size increased. So, in general the empirical Bayes and SAM methods performed better than $t$-test. 


\begin{tabular}{|c|c|clll|}
\hline \multicolumn{7}{|c|}{ nPOGR score for ALL } \\
\hline Method & subset/length & $\mathbf{5 0}$ & $\mathbf{1 0 0}$ & $\mathbf{5 0 0}$ & $\mathbf{1 0 0 0}$ \\
\hline T-test & $\mathbf{6}$ & 0.047 & 0.045 & 0.040 & 0.025 \\
& $\mathbf{1 0}$ & 0.078 & 0.100 & 0.113 & 0.091 \\
& $\mathbf{2 0}$ & 0.253 & 0.300 & 0.309 & 0.270 \\
& $\mathbf{5 0}$ & 0.567 & 0.609 & 0.617 & 0.584 \\
\hline Empirical Bayes & $\mathbf{6}$ & 0.112 & 0.129 & 0.098 & 0.078 \\
& $\mathbf{1 0}$ & 0.131 & 0.155 & 0.150 & 0.121 \\
& $\mathbf{2 0}$ & 0.292 & 0.342 & 0.331 & 0.285 \\
& $\mathbf{5 0}$ & 0.571 & 0.626 & 0.622 & 0.593 \\
\hline SAM & $\mathbf{6}$ & 0.147 & 0.151 & 0.113 & 0.099 \\
& $\mathbf{1 0}$ & 0.147 & 0.172 & 0.158 & 0.137 \\
& $\mathbf{2 0}$ & 0.339 & 0.374 & 0.349 & 0.301 \\
& $\mathbf{5 0}$ & 0.609 & 0.683 & 0.637 & 0.610 \\
\hline
\end{tabular}

Table 6. nPOGR scores for ALL data.

The nPOGR scores for the ALL data are showed in Table 6. The result for nPOGR score was similar to the nPOG score. The nPOGR scores were all similar in magnitude to the nPOG scores, reflecting the fact that normalization accounts for the strong gene-gene correlations present in this data.

Then, we studied the consistency of relatively small-scaled experiments for colon cancer with 62 samples (40 tumor samples, 22 normal samples). We detected differentially expressed genes by different sample size and length of gene lists using the three DE detection methods. We chose subsets of total size $6,12,27$ and 51 randomly and without replacement from the 40 tumor samples and 22 normal samples. The subsets were chosen in a 2:1 ratio. Then, we used different length of gene lists, 50 , 100,500 and 1000 to test the overlap between the gene lists taken from the full data and the gene lists based on the subset. The results for the POG, POGR, nPOG and nPOGR scores are given in 'Appendix A', Table 1-4. Overall, the results are similar 
to those from the ALL data, expect that the difference between the three DE detection methods is smaller.

Third, for the lung expression data, it contains 86 samples of whom 62 were alive at the end of the study and 24 had died during the study. We detected differentially expressed genes by different sample sizes and length of gene lists using the three DE detection methods. We chose total subset size of $8,12,28$ and 48 samples randomly and without replacement each from the complete dataset. The subsets were chosen in a 3:1 ratio from amongst those who lived versus those who died during the follow-up period. Then, we used different length of gene lists, 50, 100, 500 and 1000 to test the overlap genes with the gene lists taken from the full data. The results for the POG, POGR, $\mathrm{nPOG}$ and $\mathrm{nPOGR}$ scores of lung expression data are given in 'Appendix A', Tables 5-8 and are similar to the ALL and colon cancer data.

Finally, for the Leukemia data, 47 patients with acute lymphoblastic leukemia (ALL) and 25 patients with acute myeloid leukemia (AML) were monitored in the experiment. We chose total subset size of $6,12,27$ and 51 samples randomly and without replacement from the complete. The subsets were chosen in a 2:1 ratio of ALL to AML patients. Then, we used different lengths of gene lists, 50, 100, 500 and 1000 to test the overlap between gene lists based on the full data and gene lists based on the subsets. The results for the POG, POGR, nPOG and nPOGR scores of Leukemia data are given in 'Appendix A', Table 9-12. The results were similar to the three datasets above. 
Table 7 summarizes the results from the four datasets, and shows the average nPOG scores over the four lengths of the gene lists from the four datasets. Figure 1 plots to the nPOG scores listed in table 7.

\begin{tabular}{|c|c|c|c|c|}
\hline \multicolumn{5}{|c|}{ nPOG } \\
\hline \multirow{2}{*}{ Methods/Subsets } & \multicolumn{4}{|c|}{ ALL } \\
\hline & 6 & 10 & 20 & $\mathbf{5 0}$ \\
\hline T-test & 0.077 & 0.130 & 0.309 & 0.608 \\
\hline eBayes & 0.137 & 0.172 & 0.338 & 0.616 \\
\hline SAM & 0.159 & 0.184 & 0.364 & 0.646 \\
\hline \multirow{2}{*}{ Methods/Subsets } & \multicolumn{4}{|c|}{ Colon Cancer } \\
\hline & 6 & 10 & 20 & 50 \\
\hline T-test & 0.133 & 0.271 & 0.499 & 0.759 \\
\hline eBayes & 0.175 & 0.294 & 0.510 & 0.764 \\
\hline SAM & 0.182 & 0.301 & 0.528 & 0.775 \\
\hline \multirow{2}{*}{ Methods/Subsets } & \multicolumn{4}{|c|}{ Lung Expression } \\
\hline & 6 & 10 & 20 & 50 \\
\hline T-test & 0.051 & 0.098 & 0.168 & 0.332 \\
\hline eBayes & 0.069 & 0.117 & 0.179 & 0.337 \\
\hline SAM & 0.088 & 0.137 & 0.205 & 0.352 \\
\hline \multirow{2}{*}{ Methods/Subsets } & \multicolumn{4}{|c|}{ Leukemia } \\
\hline & 6 & 10 & 20 & $\mathbf{5 0}$ \\
\hline T-test & 0.176 & 0.297 & 0.572 & 0.762 \\
\hline eBayes & 0.228 & 0.326 & 0.583 & 0.767 \\
\hline SAM & 0.253 & 0.349 & 0.626 & 0.803 \\
\hline
\end{tabular}

Table 7. The summarize of the nPOG scores for four data sets used to study consistency of DE detection methods within a single data set. 


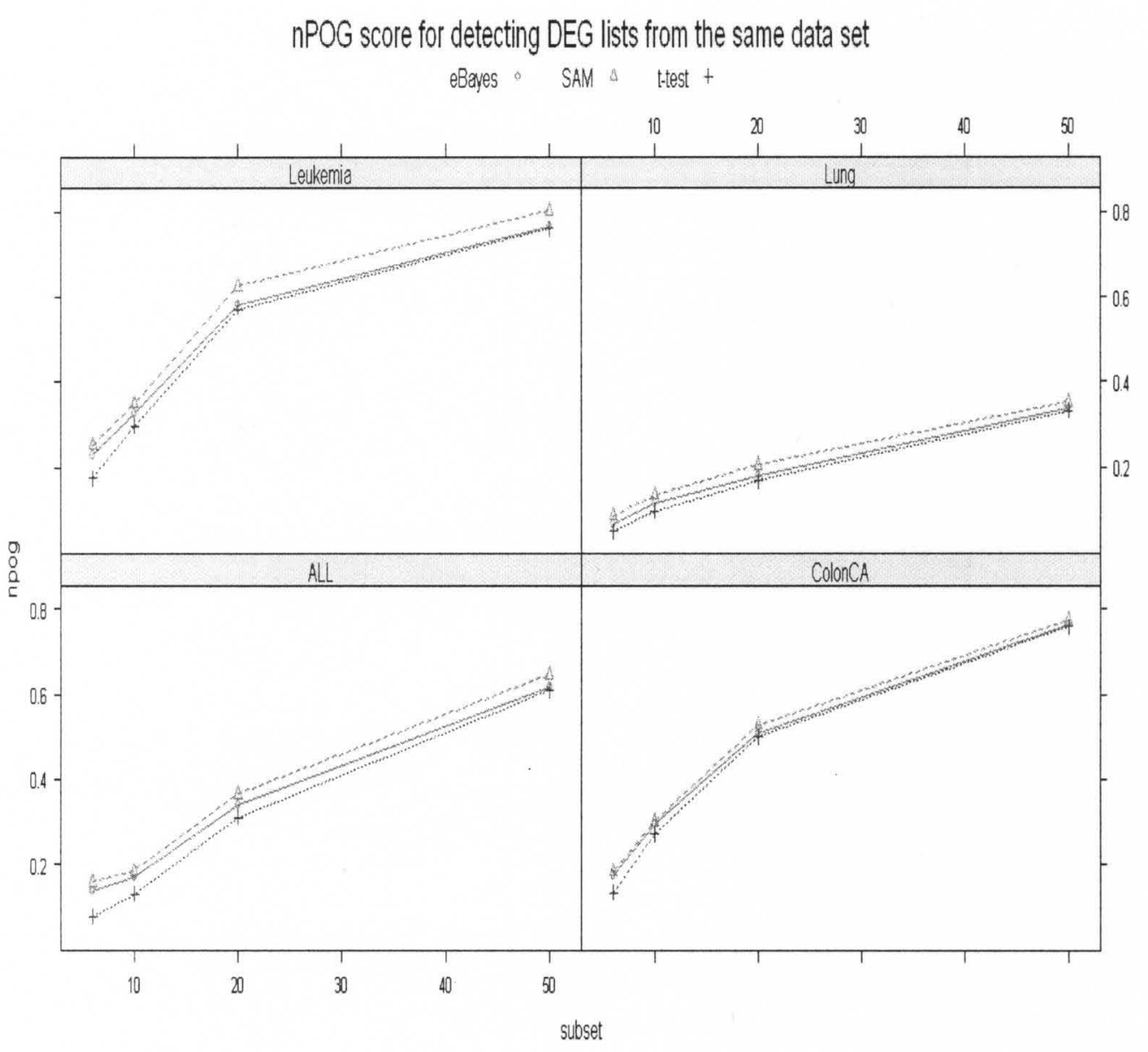

Figure 1. The figure of the nPOG scores for four data sets used to study consistency of DE detection methods within a single data set. 
Table 8 summarizes the results from the four datasets, and shows the average nPOGR scores over the four lengths of the gene lists from the four datasets. Figure 2 plots to the nPOGR scores listed in table 8 and the cutoff $=0.8$.

The nPOGR score (Table 8 )

nPOGR(cutoff $=0.8$ )

\begin{tabular}{|c|c|c|c|c|}
\hline \multirow{2}{*}{ Methods/Subsets } & \multicolumn{4}{|c|}{ ALL } \\
\hline & 6 & 10 & 20 & 50 \\
\hline T-test & 0.039 & 0.096 & 0.283 & 0.594 \\
\hline eBayes & 0.104 & 0.139 & 0.313 & 0.603 \\
\hline SAM & 0.128 & 0.153 & 0.341 & 0.635 \\
\hline \multirow{2}{*}{ Methods/Subsets } & \multicolumn{4}{|c|}{ Colon Cancer } \\
\hline & 6 & 10 & 20 & 50 \\
\hline T-test & 0 & 0 & 0.431 & 0.773 \\
\hline eBayes & 0 & 0.030 & 0.449 & 0.783 \\
\hline SAM & 0 & 0.048 & 0.473 & 0.784 \\
\hline \multirow{2}{*}{ Methods/Subsets } & \multicolumn{4}{|c|}{ Lung Expression } \\
\hline & 6 & 10 & 20 & 50 \\
\hline T-test & 0 & 0 & 0.061 & 0.249 \\
\hline eBayes & 0 & 0.014 & 0.077 & 0.257 \\
\hline SAM & 0 & 0.048 & 0.122 & 0.286 \\
\hline \multirow{2}{*}{ Methods/Subsets } & \multicolumn{4}{|c|}{ Leukemia } \\
\hline & 6 & 10 & 20 & 50 \\
\hline T-test & 0.392 & 0.515 & 0.708 & 0.851 \\
\hline eBayes & 0.455 & 0.543 & 0.718 & 0.854 \\
\hline SAM & 0.487 & 0.575 & 0.754 & 0.878 \\
\hline
\end{tabular}

Table 8 . The average of the nPOGR scores for four data sets used to study consistency of $\mathrm{DE}$ detection methods within a single data set. 


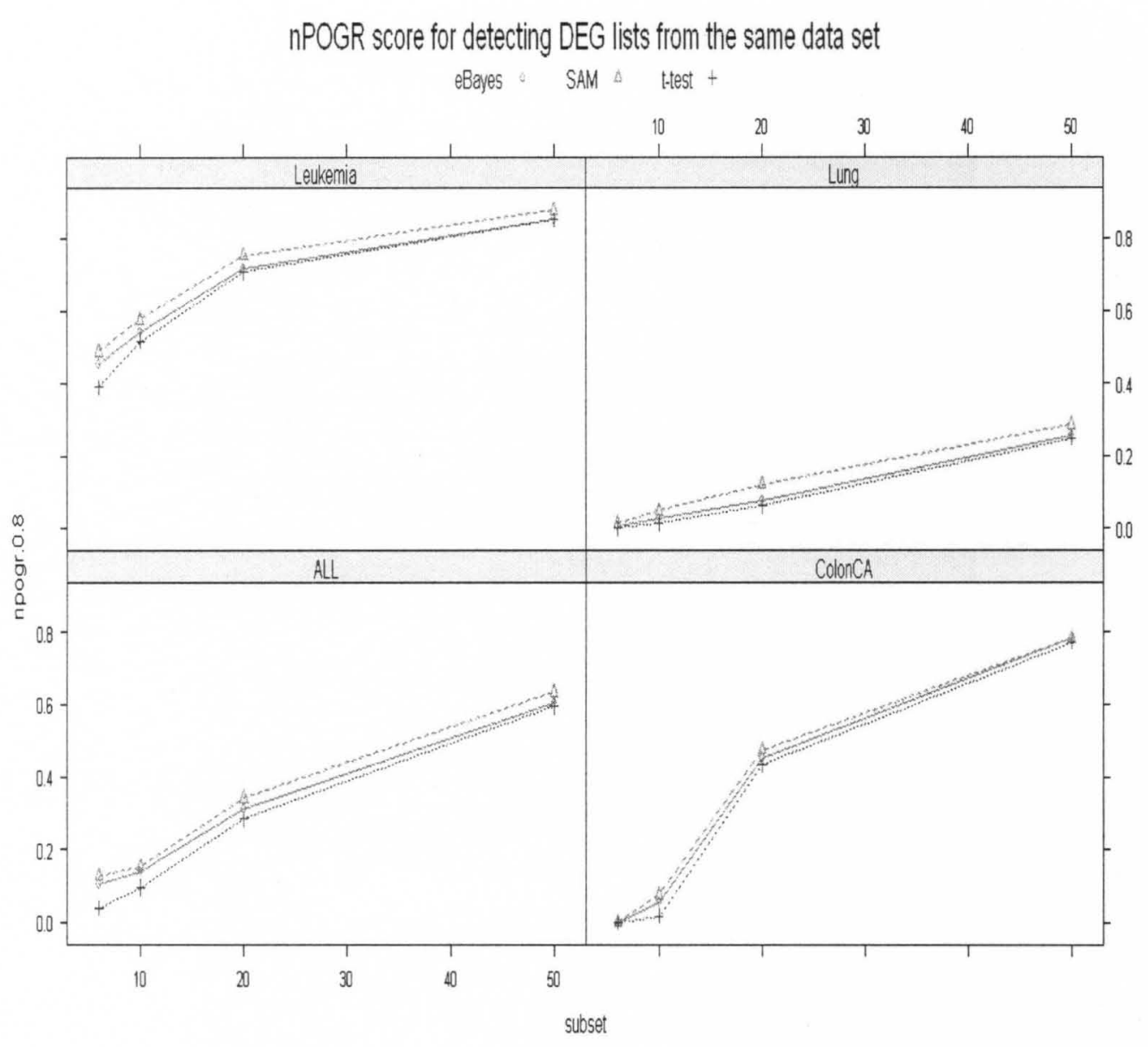

Figure 2. The plot to the nPOGR scores for four data sets in Table 8 . 


\subsection{Consistency of DE detection methods from different data sets of the same disease.}

For the six datasets of three diseases (prostate cancer, lung cancer, and DMD), we compared the differentially expressed gene lists from the two datasets for the same disease to test the consistency of the three DE detection methods.

First, for the prostate cancer data, here are 103 samples in the cDNA microarray data, which are divided into two parts, 62 tumor samples and 41 normal prostate samples. For the Affymetrix microarray data, there are 52 tumor and 50 non-tumor samples. A total of 2262 genes were found to be in common between both platforms. We considered these 2262 genes to be the complete data in both to cases, as the whole gene lists of interest. Then, we randomly picked subset of 3, 5, 10 and 25 samples from the tumor and normal tissue samples for each of the two data sets. Then, we separately used three methods to detect the differentially expressed gene lists from two subsets of the full data. Third, we use different lengths of lists 50,100, 500, 1000 to calculate the POG, POGR, nPOG and nPOGR scores. We repeated the same procedure for 100 times, and calculated the mean of the 100 scores. 


\begin{tabular}{|c|c|cccc|}
\hline \multicolumn{7}{|c|}{ nPOG score for Prostate Cancer } \\
\hline Method & subset/length & $\mathbf{5 0}$ & $\mathbf{1 0 0}$ & $\mathbf{5 0 0}$ & $\mathbf{1 0 0 0}$ \\
\hline T-test & $\mathbf{6}$ & 0.014 & 0.019 & 0.033 & 0.023 \\
& $\mathbf{1 0}$ & 0.036 & 0.036 & 0.031 & 0.023 \\
& $\mathbf{2 0}$ & 0.076 & 0.090 & 0.083 & 0.040 \\
& $\mathbf{5 0}$ & 0.085 & 0.088 & 0.085 & 0.057 \\
\hline Empirical Bayes & $\mathbf{6}$ & 0.039 & 0.049 & 0.056 & 0.027 \\
& $\mathbf{1 0}$ & 0.049 & 0.049 & 0.041 & 0.034 \\
& $\mathbf{2 0}$ & 0.086 & 0.103 & 0.090 & 0.042 \\
& $\mathbf{5 0}$ & 0.084 & 0.093 & 0.101 & 0.066 \\
\hline SAM & $\mathbf{6}$ & 0.031 & 0.041 & 0.054 & 0.028 \\
& $\mathbf{1 0}$ & 0.043 & 0.046 & 0.040 & 0.039 \\
& $\mathbf{2 0}$ & 0.083 & 0.101 & 0.090 & 0.043 \\
& $\mathbf{5 0}$ & 0.082 & 0.083 & 0.089 & 0.070 \\
\hline
\end{tabular}

Table 9. nPOG score for Prostate Cancer

\begin{tabular}{|c|c|cccc|cccc|}
\hline \multicolumn{10}{|c|}{ nPOGR 0.8 score for Prostate Cancer } \\
\hline & & \multicolumn{2}{|c|}{ From cDNA to Affymetrix } & \multicolumn{3}{|c|}{ From Affymetrix to cDNA } \\
\hline Method & subset/length & $\mathbf{5 0}$ & $\mathbf{1 0 0}$ & $\mathbf{5 0 0}$ & $\mathbf{1 0 0 0}$ & $\mathbf{5 0}$ & $\mathbf{1 0 0}$ & $\mathbf{5 0 0}$ & $\mathbf{1 0 0 0}$ \\
\hline T-test & $\mathbf{6}$ & 0.087 & 0.071 & 0.033 & 0.005 & 0.082 & 0.062 & 0.023 & 0.001 \\
& $\mathbf{1 0}$ & 0.136 & 0.104 & 0.037 & 0.007 & 0.116 & 0.092 & 0.041 & 0.006 \\
& $\mathbf{2 0}$ & 0.15 & 0.114 & 0.059 & 0.019 & 0.122 & 0.105 & 0.049 & 0.011 \\
& $\mathbf{5 0}$ & 0.351 & 0.217 & 0.099 & 0.039 & 0.333 & 0.204 & 0.089 & 0.026 \\
\hline Empirical & $\mathbf{6}$ & 0.194 & 0.188 & 0.081 & 0.009 & 0.168 & 0.188 & 0.186 & 0.128 \\
Bayes & $\mathbf{1 0}$ & 0.234 & 0.212 & 0.121 & 0.034 & 0.177 & 0.19 & 0.179 & 0.137 \\
& $\mathbf{2 0}$ & 0.394 & 0.405 & 0.223 & 0.087 & 0.188 & 0.201 & 0.193 & 0.142 \\
& $\mathbf{5 0}$ & 0.531 & 0.511 & 0.272 & 0.085 & 0.341 & 0.301 & 0.27 & 0.171 \\
\hline SAM & $\mathbf{6}$ & 0.199 & 0.183 & 0.083 & 0.014 & 0.145 & 0.165 & 0.175 & 0.122 \\
& $\mathbf{1 0}$ & 0.225 & 0.199 & 0.113 & 0.029 & 0.224 & 0.228 & 0.183 & 0.108 \\
& $\mathbf{2 0}$ & 0.366 & 0.385 & 0.211 & 0.073 & 0.243 & 0.272 & 0.242 & 0.155 \\
& $\mathbf{5 0}$ & 0.474 & 0.492 & 0.246 & 0.061 & 0.262 & 0.301 & 0.364 & 0.254 \\
\hline
\end{tabular}

Table 10. nPOGR score for Prostate Cancer with the cutoff $=0.8$.

The nPOG and nPOGR scores for the prostate cancer data are showed in Table 9 and

10 respectively. From Table 9, the nPOG scores were very low for all subset sizes, indicating low consistency between the two data sets, irrespective of the DE detective 
method. From Table 10, the nPOGR scores were generally higher than the $\mathrm{nPOG}$ scores, although there appears to be an anomaly in that the nPOGR scores decrease with the length of the list, contrary to what was expected. In both cases, the scores increased when the subsets became larger. Also, we found that, the values under the $t$-test were smaller than the values from the other two methods. It was more distinct when the number of the subsets was small. The values converged to each other when the number of the subsets increased.

For the lung cancer data, there are 18 samples in the cDNA microarray data, which are divided into two parts, 13 tumor samples and 5 normal prostate samples. For the Affymetrix microarray data, there are 21 tumor and 17 non-tumor samples. A total of 1893 genes were found to be in common between the both platforms. We considered these 1893 genes to be the complete data in both to cases. Then, we randomly picked subsets of $4,8,12$ and 16 samples from the tumor and normal tissue samples for each of the two data sets. For cDNA data, the subsets were chosen in a 3:1 ratio of tumor to normal samples. For Affymetrix data the subsets were chosen in a 1:1 ratio of tumor to normal samples. Then, we separately used three methods to detect the differentially expressed gene lists from two subsets of the full data. Third, we used different lengths of lists 50,100,500, 1000 to calculate the scores. At last, we repeated the same procedure 100 times, and calculated the mean of the 100 scores. The results for the nPOG and nPOGR scores of lung cancer data are given in 'Appendix A', Table 13-14. The scores are generally higher compared to the prostate cancer data, and the differences between the $t$-test and the other two methods are more pronounced, for the nPOG score. The rest results are similar to the prostate cancer data sets. 
For the duchenne muscular dystrophy (DMD) data, there are 24 samples in the first Affymetrix data, which are divided into 12 DMD patient samples and 12 control samples (Haslett et al., 2002). For the other Affymetrix there are 22 DMD patient samples and 14 control samples (Pescatori et al., 2007). A total of 3158 genes were founded to be common between both data sets. We considered these 3158 genes to be the complete data in both data sets. Then, we randomly picked subsets of $6,8,12$ and 24 samples for each of the data sets without replacement. For Haslett's data, the subsets were chosen in a ratio of $1: 1$ between patients and normal samples, and the subsets were selected in a ratio of $2: 1$ between patients and controls in Pescatori's data. Then, we separately used three methods to detect the differentially expressed gene lists from two subsets of the full data. Third, we used different lengths of lists 50 , $100,500,1000$ to calculate the POG, POGR, nPOG and nPOGR scores. We repeated the procedure 100 times, and calculated the mean of the 100 scores. The results for the nPOG and nPOGR scores of lung cancer data are given in 'Appendix A', Table 15-16.The result was almost same to the lung cancer data sets.

Table 11 and 12 give the average nPOG and nPOGR scores for each of the three data sets, covered over the four subsets sizes. Figure 3 plots the nPOG scores from Table 11, while Figures 5 and 6 plot the nPOGR scores from Table 12. 


\begin{tabular}{|c|c|c|c|c|}
\hline \multirow{2}{*}{ Methods/Subsets } & \multicolumn{4}{|c|}{ Prostate Cancer } \\
\hline & 6 & 10 & 20 & $\mathbf{5 0}$ \\
\hline T-test & 0.022 & 0.032 & 0.072 & 0.079 \\
\hline eBayes & 0.043 & 0.043 & 0.080 & 0.086 \\
\hline SAM & 0.039 & 0.042 & 0.079 & 0.081 \\
\hline \multirow{2}{*}{ Methods/Subsets } & \multicolumn{4}{|c|}{ Lung Cancer } \\
\hline & 4 & 8 & 12 & 16 \\
\hline T-test & 0.144 & 0.152 & 0.195 & 0.224 \\
\hline eBayes & 0.205 & 0.208 & 0.240 & 0.260 \\
\hline SAM & 0.210 & 0.214 & 0.248 & 0.273 \\
\hline \multirow{2}{*}{ Methods/Subsets } & \multicolumn{4}{|c|}{ DMD } \\
\hline & 6 & 12 & 18 & 24 \\
\hline T-test & 0.119 & 0.265 & 0.342 & 0.400 \\
\hline eBayes & 0.251 & 0.335 & 0.389 & 0.447 \\
\hline SAM & 0.240 & 0.335 & 0.395 & 0.449 \\
\hline
\end{tabular}

Table 11. The summarize of the nPOG scores for six data sets used to study consistency of DE detection methods between different data sets of the same disease.

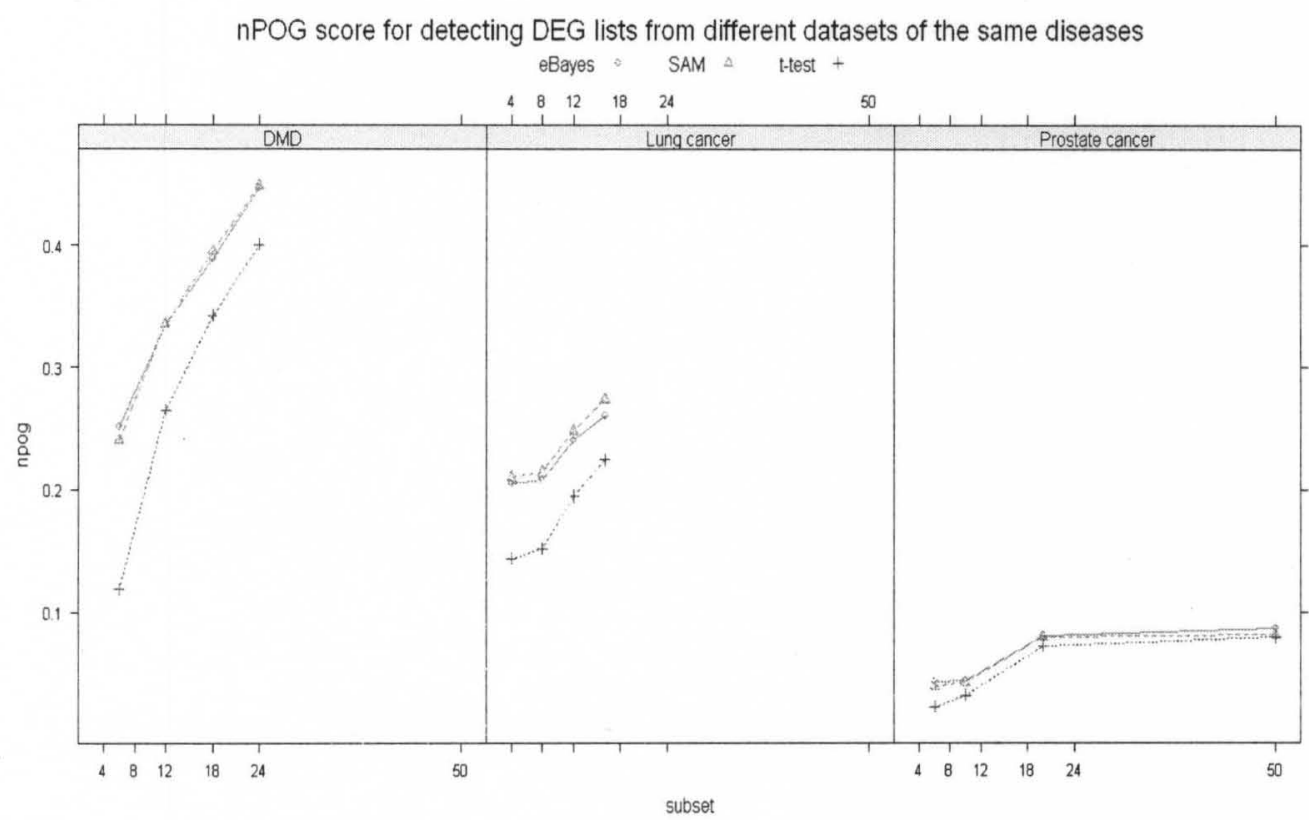

Figure 3. The plot of the nPOG scores for six data sets in Table 11. 
nPOGR 0.75

\begin{tabular}{|c|c|c|c|c|c|c|c|c|}
\hline \multirow{3}{*}{ Methods/Subsets } & \multicolumn{4}{|c|}{ From Data A to Data B } & \multicolumn{4}{|c|}{ From Data B to Data A } \\
\hline & \multicolumn{4}{|c|}{ Prostate Cancer } & \multicolumn{4}{|c|}{ Prostate Cancer } \\
\hline & 6 & 10 & 20 & $\mathbf{5 0}$ & 6 & 10 & 20 & 50 \\
\hline T-test & 0.049 & 0.071 & 0.086 & 0.177 & 0.042 & 0.064 & 0.072 & 0.163 \\
\hline eBayes & 0.118 & 0.15 & 0.277 & 0.35 & 0.168 & 0.171 & 0.181 & 0.271 \\
\hline SAM & 0.12 & 0.142 & 0.259 & 0.318 & 0.152 & 0.186 & 0.228 & 0.295 \\
\hline \multirow{2}{*}{ Methods/Subsets } & \multicolumn{4}{|c|}{ Lung Cancer } & \multicolumn{4}{|c|}{ Lung Cancer } \\
\hline & 4 & 8 & 12 & 16 & 4 & 8 & 12 & 16 \\
\hline T-test & 0.064 & 0.081 & 0.139 & 0.196 & 0.081 & 0.099 & 0.148 & 0.196 \\
\hline eBayes & 0.332 & 0.339 & 0.378 & 0.406 & 0.286 & 0.294 & 0.34 & 0.358 \\
\hline SAM & 0.339 & 0.345 & 0.387 & 0.409 & 0.292 & 0.302 & 0.34 & 0.359 \\
\hline \multirow{2}{*}{ Methods/Subsets } & \multicolumn{4}{|c|}{ DMD } & \multicolumn{4}{|c|}{ DMD } \\
\hline & 6 & 12 & 18 & 24 & 6 & 12 & 18 & 24 \\
\hline T-test & 0.158 & 0.185 & 0.213 & 0.263 & 0.16 & 0.191 & 0.254 & 0.285 \\
\hline eBayes & 0.472 & 0.545 & 0.585 & 0.61 & 0.485 & 0.565 & 0.613 & 0.656 \\
\hline SAM & 0.461 & 0.543 & 0.585 & 0.603 & 0.466 & 0.558 & 0.612 & 0.655 \\
\hline
\end{tabular}

Table 12. The summarize of the nPOGR scores for six data sets used to study

consistency of DE detection methods between different data sets of the same disease, with the cutoff $=0.75$. 


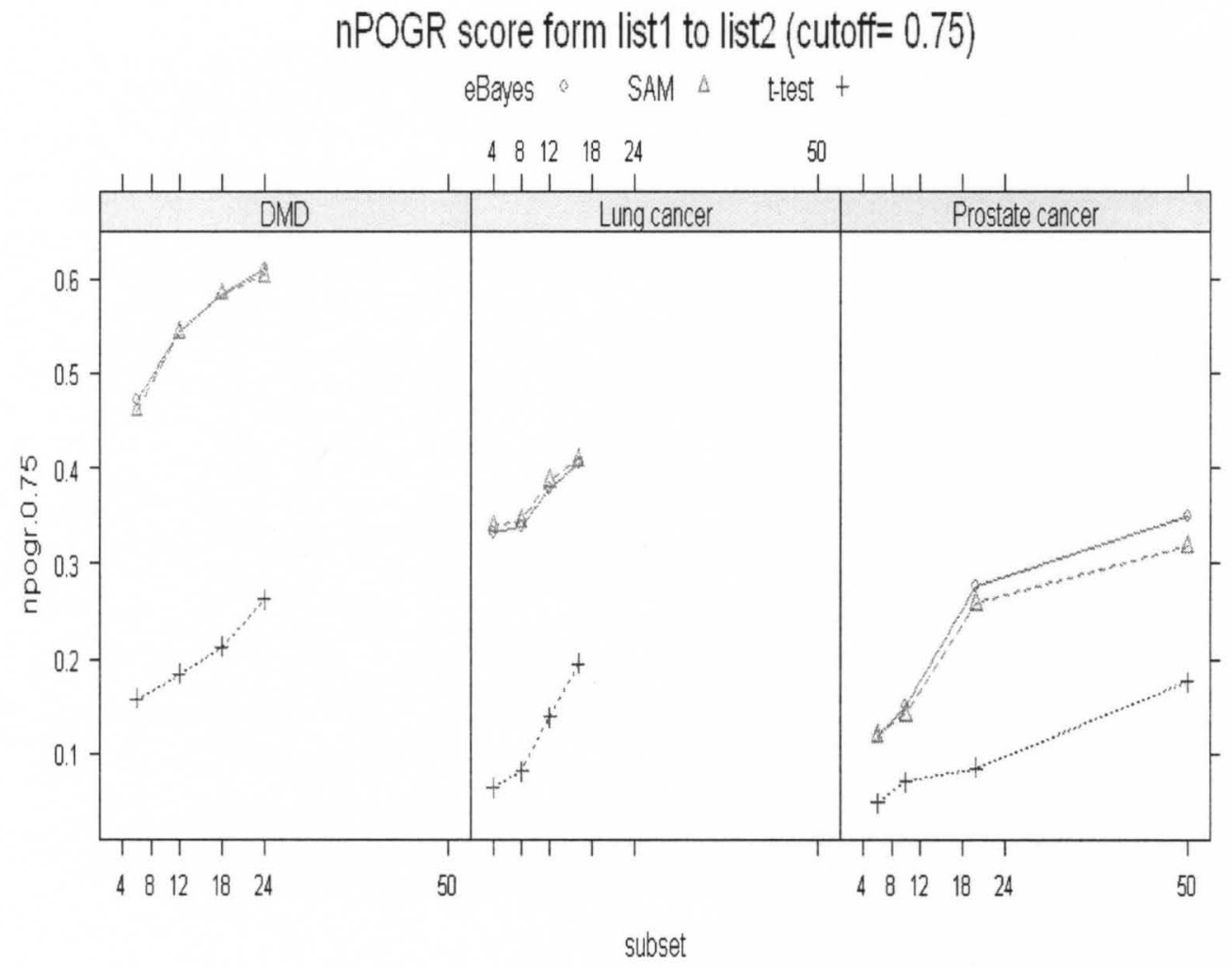

Figure 4. The plot of the nPOGR scores getting from list 1 to list 2 for six data sets in Table 12 


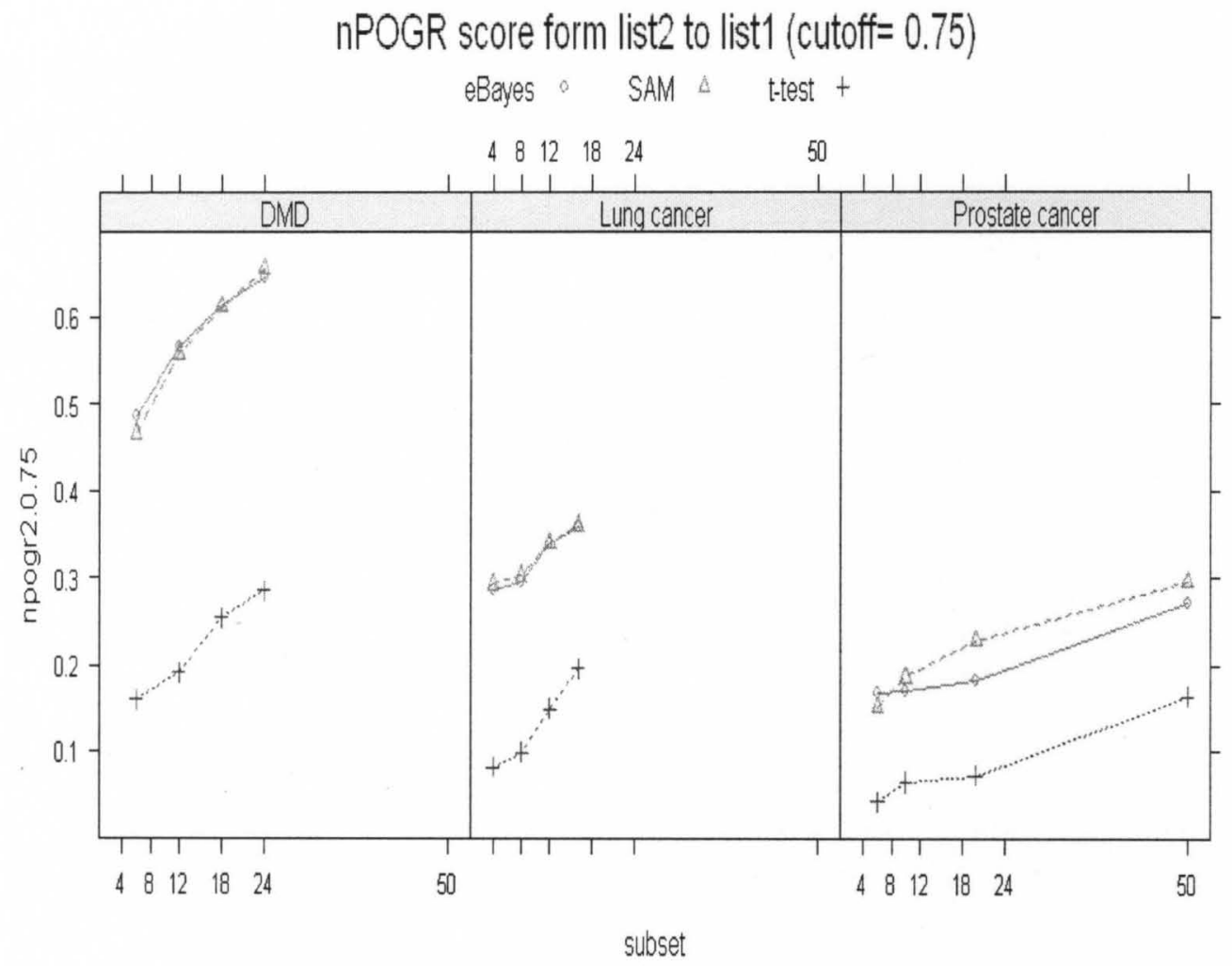

Figure 5. The plot of the nPOGR scores from list 2 to list 1 for six data sets in Table 12. 


\section{CHAPTER IV}

\section{DISCUSSION}

In our article, three distinct methods were used for selecting differentially expressed genes, the $t$-test, empirical Bayes and SAM. The calculation of the test statistic for each method captures different aspects of the changes in expression measurements for each gene, resulting in potentially different lists of DE genes. The traditional $t$-test still remains a popular choice, and we compared the $t$-test with the empirical Bayes and SAM methods to evaluate the empirical impact of choice of test-statistic on the consistency of the resulting list of differentially expressed genes. Of particular interest was the consistency of each method when the available sample size was small.

Based on our results, the traditional $t$-statistic had the poorest performance relative to SAM and the empirical Bayesian methods. This was particularly evident in the studies involving two data sets for the same disease, although less evident for the studies involving subsets of a single data set. The $t$-statistic can be unstable in small data sets, because the variance estimate can be quite skewed by outlying expression values. Further, due to the large numbers of genes in microarray datasets, there will always be some genes with low standard deviation by chance. In this case, 
these genes will have large t-statistics and would be considered to be differentially expressed falsely. In this case, the empirical Bayes and SAM statistics, which can be considered as modified $t$-statistic methods, gave better results.

When the length of the gene lists increased, the POG and POGR scores generally increased. But for the nPOG and nPOGR scores, the values of the scores did not change uniformly with the length of the lists. and the degree of change was smaller than for the POG and POGR scores. So, the POG and POGR scores are more strongly affected by the length of the gene list, and the nPOG and nPOGR scores are more appropriate to evaluate the consistency of DE detection methods with different lengths of lists. Next, we found that all the scores increased when the size of the subsets increased. We can conclude that the sample size is important when evaluating the consistency of the DE detection methods. In the first part of the experiment, we compared the differentially expressed gene lists from different subsamples with the full data in the same study to avoid any platform and site differences. According to our result, the differentially expressed gene lists can still be very inconsistent, especially when the subsets were small.

The DE gene lists from small-scaled microarray studies may only contain a small portion of the total number of differentially expressed genes in a disease, so the POG scores will generally be low. However, each differentially expressed gene list may include mostly true genes. Usually, the global expression changes of genes in a disease may introduce great uncertainty to the findings at the individual gene level (Jeffery 2006). In this case, even if there are many 'true' differentially expressed genes in a disease, we may be only discovering the most significant ones. Thus, the POGR score is helpful for measuring how well different methods for detecting DE genes retain the biologically important genes, particularly when considering small 
subsets of the samples. As the result, when the sample size of the microarray study is small, the nPOGR score may give the best result.

According to the result we got, we used the nPOG and nPOGR scores to evaluate the efficacy of the methods. These three methods were then applied to ten real microarray data sets. Our results indicated that SAM was the most consistent method in the analysis of all the data sets, although the empirical Bayes method performed similarly to SAM. When the sample size of the subset was small ( 3 arrays per group), the resulting gene list based on all three mehods had a low consistency with the gene list based on the full data, although the empirical Bayes and SAM methods had higher consistencies relative to the $t$-test.

This disparity between the methods is more apparent when considering the studies using two different data sets for the same disease. In this case, both the SAM and empirical Bayes methods had considerably higher consistencies relative to the t-test. In light of all the evidence, we recommend that investigators use these moderated versions in lieu of the $t$-test when the sample size is small (less than 5 per group).

There are several limitations with our study that are planned as areas of future research. First, our findings suggest that the moderated versions of the $t$-test are more consistent than the traditional $t$-test for microarray studies. One aspect which we did not explicitly evaluate is how robust each of the methods are to departures from normality. Both SAM and the empirical Bayes $t$-test are moderated by adding a strictly positive constant to the denominator of the ordinary $t$-statistic. The addition of this constant reduces the chance of 'detecting' genes (declaring them to be statistically significant) which have a low standard deviation by chance. Since common departures from normality (skewness and/or outliers) would inflate the sample variance, this suggests that the moderated versions of the $t$-test would be more robust in this case. 
However, an explicit study involving non-normal real and/or simulated data would be needed to test this hypothesis.

In our study design we ignored design factors which could cause dependencies between samples from the same phenotypic group (diseased / control). This may have an impact on the results, and in particular a sampling design which accounts for the design factors in the study may be more appropriate. Additionally, using a more complicated model for the gene expression values, which includes the original design variables in the model, would be worthwhile to investigate. In this case, a comparison between the standard linear model and the hierarchical empirical Bayesian model (Smyth 2004) would be warranted. In subsequent research, we will check the experimental design for each data set, to determine whether this should be accounted for when taking subsamples from the data.

In our paper, we randomly chose subsets from the full data set and evaluated consistency between the DE detection methods using fixed list lengths. The smallest subset size we used was 6 ( 3 in each group), and sample sizes this small will have correspondingly low power to detect DE genes. Since we used fixed list lengths when evaluating the consistency for each method, our scores will only reflect the consistency based on the test statistic itself, and ignores the ability of each method to retain power when the sample size is small. Subsequent studies which compare lists based on a significance threshold would be needed to test whether any differences between the methods existed on this account.

Lastly, in our research we evaluated the consistency of the DE detection methods using only real data. To supplement these experiments, it would be interesting to use simulation methods (Parrish 2009) to create simulated data which reflect the real microarray data but with a larger number of samples. This would 
enable us to better determine a 'true' list of DE genes, and to better map the rate of convergence between the methods as the sample size increases. 


\section{REFERENCES}

Alon, U., N, Baska., D, A, Notterman., K,Gish., S,Ybarra., D,Mack., And A,J, Levine (1999). "Broad patterns of gene expression revealed by clustering analysis of tumor and normal colon tissues probed by oligonucleotide arrays." Proc. Natl. Acad. Sci. USA 96: 6745-6750.

Baldi, P., Anthony, D. Long (2001). "A Bayesian framework for the analysis of microarray expression data: regularized $t$-test and statistical inferences of gene changes." Bioinformatics 17(6): 509-519

Beer, D. G., Kardia, Sharon L. R., Huang, Chiang-Ching., Giordano, Thomas J., Levin, Albert M., Misek, David E., Lin, Lin., Chen, Guoan., Gharib, Tarek G., Thomas, Dafydd G., Lizyness, Michelle L., Kuick, Rork., Hayasaka, Satoru., Taylor, Jeremy M. G., Iannettoni, Mark D., Orringer, Mark B., Hanash, Samir (2002). "Gene-expression profiles predict survival of patients with lung adenocarcinoma." Nature Medicine 8(8): 816-824.

Bhattacharjee, A. (2001). "Classification of human lung carcinomas by mRNA expression profiling reveals distinct adenocarcinoma subclasses." Proceedings of the National Academy of Sciences 98(24): 13790-13795.

Chen, J. J., Hsueh, Huey-Miin., Delongchamp, Robert R., Lin, Chien-Ju., Tsai, Chen-An (2007). "Reproducibility of microarray data: a further analysis of microarray quality control (MAQC) data." BMC Bioinformatics 8(1): 412.

Efron, B. (2009). "Empirical Bayes Estimates for Large-Scale Prediction Problems." Journal of the American Statistical Association 104(487): 1015-1028.

Garber, M. E. (2001). "Diversity of gene expression in adenocarcinoma of the lung." Proceedings of the National Academy of Sciences 98(24): 13784-13789.

Golub, T., R., D, K, Slonim., P, Tamayo., C, Huard., M, Gaasenbeek., J, P, Mesirov., H, Coller., M,L, Loh., J, R, Downing., M, A,Caligiuri., C, D, Bloomfield., E, S,Lander. (1999). "Molecular Classification of Cancer: Class Discovery and Class Prediction by Gene Expression Monitoring." Science 286(5439): 531-537.

Haslett, J. N. (2002). "Gene expression comparison of biopsies from Duchenne muscular dystrophy (DMD) and normal skeletal muscle." Proceedings of the National Academy of Sciences 99(23): 15000-15005. 
Hong, F. and R. Breitling (2008). "A comparison of meta-analysis methods for detecting differentially expressed genes in microarray experiments." Bioinformatics 24(3): 374-382.

Jeffery, I. B., Higgins, Desmond G., Culhane, Aedín C. (2006). "Comparison and evaluation of methods for generating differentially expressed gene lists from microarray data." BMC Bioinformatics 7(1): 359 .

Lapointe, J. (2004). "Gene expression profiling identifies clinically relevant subtypes of prostate cancer." Proceedings of the National Academy of Sciences 101(3): 811-816.

McCarthy, D. J. and G. K. Smyth (2009). "Testing significance relative to a fold-change threshold is a TREAT." Bioinformatics 25(6): 765-771.

Parrish, R. S., Spencer Iii, Horace J., Xu, Ping (2009). "Distribution modeling and simulation of gene expression data." Computational Statistics \& Data Analysis 53(5): 1650-1660.

Pescatori, M., Broccolini, A., Minetti, C., Bertini, E., Bruno, C., D'Amico, A., Bernardini, C., Mirabella, M., Silvestri, G., Giglio, V., Modoni, A., Pedemonte, M., Tasca, G., Galluzzi, G., Mercuri, E., Tonali, P. A., Ricci, E. (2007). "Gene expression profiling in the early phases of DMD: a constant molecular signature characterizes DMD muscle from early postnatal life throughout disease progression." The FASEB Journal 21(4): 1210-1226.

Sabina, C., Xiaochun, Li., Robert, Gentleman., Antonella, Vitale., Marco, Vignetti., Franco, Mandelli.,Jerome, Ritz., and Robin, Foa. (2004). "Gene expression profile of adult T-cell acute lymphocytic leukemia identifies distinct subsets of patients with different response to therapy and survival." Blood 103(7): 2771-2778.

Shi, L., Reid, Laura H.. Jones, Wendell D., Shippy, Richard., Warrington, Janet A., Baker, Shawn C., Collins, Patrick J., de Longueville, Francoise. (2006). "The MicroArray Quality Control (MAQC) project shows inter- and intraplatform reproducibility of gene expression measurements." Nature Biotechnology 24(9): 1151-1161.

Singh, D., Phillip, G, Febbo., Kenneth, Ross., Donald, G, Jackson., Judith, Manola., Christine, Ladd., Pablo, Tamayo., Andrew ,A, Renshaw., Anthony, V, D'Amico., Jerome, P, Richie., Eric, S, Lander., Massimo, Loda., Philip, W, Kantoff., Todd, R, Golub., William, R, Sellers. (2002). "Gene expression correlates of clinical prostate cancer behavior." Cancer Cell. 2002 Mar;1(2):203-9.

Smyth, G. (2004). "Linear models and empirical Bayes methods for assessing differential expression in Microarray Experiments." Stat Appl Genet Mol Biol 3(1): Article 3.

Smyth, G. (2005). "Limma: Linear Models for Microarray Data." Bioinformatics and Computational Biology Solutions using R and Bioconductor. Edited by Gentleman R, Carey V, Dudoit S, Irizarry R, Huber W, New York: Springer: 397-420. 
Smyth, G. K., Michaud, J., Scott, H. S. (2005). "Use of within-array replicate spots for assessing differential expression in microarray experiments." Bioinformatics 21(9): 2067-2075.

Tu, Y. (2002). "Quantitative noise analysis for gene expression microarray experiments." Proceedings of the National Academy of Sciences 99(22): 14031-14036.

Tusher, V., Goss., Robert, Tibshirani., Gilbert Chu (2001). "Significance analysis of microarrays applied to the ionizing radiation response." Proceedings of the National Academy of Sciences 98(9): 5116-5121.

Xu, P., Brock, Guy N., Parrish, Rudolph S. (2009). "Modified linear discriminant analysis approaches for classification of high-dimensional microarray data." Computational Statistics \& Data Analysis 53(5): 1674-1687.

Zhang, M., Zhang, L., Zou, J., Yao, C., Xiao, H., Liu, Q., Wang, J., Wang, D., Wang, C., Guo, $Z$. (2009). "Evaluating reproducibility of differential expression discoveries in microarray studies by considering correlated molecular changes." Bioinformatics 25(13): 1662-1668. 


\section{APPENDIX A}

Tables

\begin{tabular}{|c|c|clll|}
\hline \multicolumn{7}{|c|}{ POG score for Colon Cancer } \\
\hline Method & subset/length & $\mathbf{5 0}$ & $\mathbf{1 0 0}$ & $\mathbf{5 0 0}$ & $\mathbf{1 0 0 0}$ \\
\hline T-test & $\mathbf{6}$ & 0.140 & 0.170 & 0.380 & 0.560 \\
& $\mathbf{1 0}$ & 0.270 & 0.298 & 0.489 & 0.627 \\
& $\mathbf{2 0}$ & 0.502 & 0.524 & 0.663 & 0.729 \\
& $\mathbf{5 0}$ & 0.761 & 0.784 & 0.841 & 0.860 \\
\hline Empirical Bayes & $\mathbf{6}$ & 0.175 & 0.223 & 0.416 & 0.574 \\
& $\mathbf{1 0}$ & 0.299 & 0.318 & 0.507 & 0.635 \\
& $\mathbf{2 0}$ & 0.518 & 0.540 & 0.668 & 0.731 \\
& $\mathbf{5 0}$ & 0.770 & 0.790 & 0.844 & 0.861 \\
\hline SAM & $\mathbf{6}$ & 0.179 & 0.234 & 0.420 & 0.577 \\
& $\mathbf{1 0}$ & 0.301 & 0.332 & 0.515 & 0.637 \\
& $\mathbf{2 0}$ & 0.538 & 0.568 & 0.677 & 0.736 \\
& $\mathbf{5 0}$ & 0.793 & 0.797 & 0.847 & 0.865 \\
\hline
\end{tabular}

Table 1

\begin{tabular}{|c|c|cccc|}
\hline \multicolumn{7}{|c|}{ POGR score for Colon Cancer } \\
\hline Method & subset/length & $\mathbf{5 0}$ & $\mathbf{1 0 0}$ & $\mathbf{5 0 0}$ & $\mathbf{1 0 0 0}$ \\
\hline T-test & $\mathbf{6}$ & 0.410 & 0.512 & 0.807 & 0.907 \\
& $\mathbf{1 0}$ & 0.518 & 0.633 & 0.882 & 0.935 \\
& $\mathbf{2 0}$ & 0.796 & 0.829 & 0.930 & 0.956 \\
& $\mathbf{5 0}$ & 0.934 & 0.944 & 0.973 & 0.978 \\
\hline Empirical Bayes & $\mathbf{6}$ & 0.482 & 0.599 & 0.832 & 0.916 \\
& $\mathbf{1 0}$ & 0.578 & 0.659 & 0.890 & 0.936 \\
& $\mathbf{2 0}$ & 0.810 & 0.841 & 0.931 & 0.955 \\
& $\mathbf{5 0}$ & 0.939 & 0.952 & 0.973 & 0.978 \\
\hline SAM & $\mathbf{6}$ & 0.495 & 0.602 & 0.833 & 0.914 \\
& $\mathbf{1 0}$ & 0.597 & 0.670 & 0.892 & 0.935 \\
& $\mathbf{2 0}$ & 0.832 & 0.858 & 0.933 & 0.954 \\
& $\mathbf{5 0}$ & 0.958 & 0.949 & 0.973 & 0.977 \\
\hline
\end{tabular}

Table 2 


\begin{tabular}{|c|c|cccc|}
\hline \multicolumn{7}{|c|}{ nPOG score for Colon Cancer } \\
\hline Method & subset/length & $\mathbf{5 0}$ & $\mathbf{1 0 0}$ & $\mathbf{5 0 0}$ & $\mathbf{1 0 0 0}$ \\
\hline T-test & $\mathbf{6}$ & 0.118 & 0.124 & 0.170 & 0.121 \\
& $\mathbf{1 0}$ & 0.249 & 0.261 & 0.319 & 0.255 \\
& $\mathbf{2 0}$ & 0.489 & 0.499 & 0.551 & 0.457 \\
& $\mathbf{5 0}$ & 0.755 & 0.772 & 0.787 & 0.720 \\
\hline Empirical Bayes & $\mathbf{6}$ & 0.154 & $\mathbf{0 . 1 8 1}$ & 0.218 & 0.149 \\
& $\mathbf{1 0}$ & 0.280 & 0.282 & 0.343 & 0.270 \\
& $\mathbf{2 0}$ & 0.505 & 0.516 & 0.558 & 0.462 \\
& $\mathbf{5 0}$ & 0.765 & 0.778 & 0.790 & 0.723 \\
\hline SAM & $\mathbf{6}$ & 0.158 & 0.192 & 0.224 & 0.154 \\
& $\mathbf{1 0}$ & 0.282 & 0.296 & 0.354 & 0.274 \\
& $\mathbf{2 0}$ & 0.525 & 0.546 & 0.569 & 0.473 \\
& $\mathbf{5 0}$ & 0.788 & 0.786 & 0.795 & 0.729 \\
\hline
\end{tabular}

Table 3

\begin{tabular}{|c|c|cccc|}
\hline \multicolumn{7}{|c|}{ nPOGR score for Colon Cancer } \\
\hline Method & subset/length & $\mathbf{5 0}$ & $\mathbf{1 0 0}$ & $\mathbf{5 0 0}$ & $\mathbf{1 0 0 0}$ \\
\hline T-test & $\mathbf{6}$ & 0 & 0 & 0 & 0 \\
& $\mathbf{1 0}$ & 0 & 0 & 0.074 & 0 \\
& $\mathbf{2 0}$ & 0.560 & 0.496 & 0.437 & 0.232 \\
& $\mathbf{5 0}$ & 0.859 & 0.836 & 0.782 & 0.614 \\
\hline Empirical Bayes & $\mathbf{6}$ & 0 & 0 & 0 & 0 \\
& $\mathbf{1 0}$ & 0.103 & 0 & 0.130 & 0 \\
& $\mathbf{2 0}$ & 0.589 & 0.531 & 0.446 & 0.228 \\
& $\mathbf{5 0}$ & 0.871 & 0.859 & 0.787 & 0.612 \\
\hline SAM & $\mathbf{6}$ & 0 & 0 & 0 & 0 \\
& $\mathbf{1 0}$ & 0.144 & 0.021 & 0.146 & 0 \\
& $\mathbf{2 0}$ & 0.638 & 0.583 & 0.462 & 0.211 \\
& $\mathbf{5 0}$ & 0.910 & 0.851 & 0.781 & 0.596 \\
\hline
\end{tabular}

Table 4 


\begin{tabular}{|c|c|clll|}
\hline \multicolumn{7}{|c|}{ POG score for Lung Expression } & & & \\
\hline Method & subset/length & $\mathbf{5 0}$ & $\mathbf{1 0 0}$ & $\mathbf{5 0 0}$ & $\mathbf{1 0 0 0}$ \\
\hline T-test & $\mathbf{6}$ & 0.064 & 0.079 & 0.209 & 0.346 \\
& $\mathbf{1 0}$ & 0.099 & 0.127 & 0.253 & 0.378 \\
& $\mathbf{2 0}$ & 0.182 & 0.204 & 0.311 & 0.416 \\
& $\mathbf{5 0}$ & 0.341 & 0.364 & 0.455 & 0.519 \\
\hline Empirical Bayes & $\mathbf{6}$ & 0.088 & 0.105 & 0.220 & 0.352 \\
& $\mathbf{1 0}$ & 0.130 & 0.156 & 0.261 & 0.382 \\
& $\mathbf{2 0}$ & 0.202 & 0.218 & 0.315 & 0.417 \\
& $\mathbf{5 0}$ & 0.351 & 0.374 & 0.456 & 0.518 \\
\hline SAM & $\mathbf{6}$ & 0.102 & 0.123 & 0.237 & 0.367 \\
& $\mathbf{1 0}$ & 0.151 & 0.180 & 0.273 & 0.393 \\
& $\mathbf{2 0}$ & 0.232 & 0.254 & 0.336 & 0.427 \\
& $\mathbf{5 0}$ & 0.377 & 0.388 & 0.462 & 0.528 \\
\hline
\end{tabular}

Table 5

\begin{tabular}{|c|c|clll|}
\hline \multicolumn{7}{|c|}{ POGR score for Lung Expression } \\
\hline Method & subset/length & $\mathbf{5 0}$ & $\mathbf{1 0 0}$ & $\mathbf{5 0 0}$ & $\mathbf{1 0 0 0}$ \\
\hline T-test & $\mathbf{6}$ & 0.065 & 0.080 & 0.258 & 0.443 \\
& $\mathbf{1 0}$ & 0.100 & 0.128 & 0.302 & 0.475 \\
& $\mathbf{2 0}$ & 0.183 & 0.206 & 0.346 & 0.499 \\
& $\mathbf{5 0}$ & 0.343 & 0.368 & 0.487 & 0.593 \\
\hline Empirical Bayes & $\mathbf{6}$ & 0.090 & 0.108 & 0.272 & 0.450 \\
& $\mathbf{1 0}$ & 0.132 & 0.158 & 0.311 & 0.481 \\
& $\mathbf{2 0}$ & 0.204 & 0.221 & 0.353 & 0.505 \\
& $\mathbf{5 0}$ & 0.353 & 0.381 & 0.489 & 0.594 \\
\hline SAM & $\mathbf{6}$ & 0.104 & 0.132 & 0.304 & 0.474 \\
& $\mathbf{1 0}$ & 0.154 & 0.188 & 0.337 & 0.502 \\
& $\mathbf{2 0}$ & 0.234 & 0.260 & 0.391 & 0.530 \\
& $\mathbf{5 0}$ & 0.379 & 0.398 & 0.510 & 0.614 \\
\hline
\end{tabular}

Table 6 


\begin{tabular}{|c|c|llll|}
\hline \multicolumn{7}{|c|}{ nPOG score for Lung Expression } \\
\hline Method & subset/length & $\mathbf{5 0}$ & $\mathbf{1 0 0}$ & $\mathbf{5 0 0}$ & $\mathbf{1 0 0 0}$ \\
\hline T-test & $\mathbf{6}$ & 0.050 & 0.050 & 0.059 & 0.043 \\
& $\mathbf{1 0}$ & 0.086 & 0.099 & 0.115 & 0.093 \\
& $\mathbf{2 0}$ & 0.166 & 0.178 & 0.182 & 0.147 \\
& $\mathbf{5 0}$ & 0.330 & 0.343 & 0.354 & 0.299 \\
\hline Empirical Bayes & $\mathbf{6}$ & 0.074 & 0.077 & 0.073 & 0.052 \\
& $\mathbf{1 0}$ & 0.118 & 0.129 & 0.125 & 0.098 \\
& $\mathbf{2 0}$ & 0.187 & 0.192 & 0.187 & 0.149 \\
& $\mathbf{5 0}$ & 0.341 & 0.353 & 0.355 & 0.297 \\
\hline SAM & $\mathbf{6}$ & 0.089 & 0.095 & 0.093 & 0.074 \\
& $\mathbf{1 0}$ & 0.139 & 0.154 & 0.139 & 0.115 \\
& $\mathbf{2 0}$ & 0.218 & 0.229 & 0.211 & 0.164 \\
& $\mathbf{5 0}$ & 0.367 & 0.367 & 0.361 & 0.311 \\
\hline
\end{tabular}

Table 7

\begin{tabular}{|c|c|cccc|}
\hline \multicolumn{7}{|c|}{ nPOGR score for Lung Expression } \\
\hline Method & subset/length & $\mathbf{5 0}$ & $\mathbf{1 0 0}$ & $\mathbf{5 0 0}$ & $\mathbf{1 0 0 0}$ \\
\hline T-test & $\mathbf{6}$ & 0 & 0 & 0 & 0 \\
& $\mathbf{1 0}$ & 0.036 & 0.016 & 0 & 0 \\
& $\mathbf{2 0}$ & 0.122 & 0.097 & 0.020 & 0.006 \\
& $\mathbf{5 0}$ & 0.294 & 0.284 & 0.228 & 0.190 \\
\hline Empirical Bayes & $\mathbf{6}$ & 0.023 & 0 & 0 & 0 \\
& $\mathbf{1 0}$ & 0.070 & 0.050 & 0 & 0 \\
& $\mathbf{2 0}$ & 0.144 & 0.114 & 0.031 & 0.017 \\
& $\mathbf{5 0}$ & 0.305 & 0.299 & 0.231 & 0.192 \\
\hline SAM & $\mathbf{6}$ & 0.038 & 0.017 & 0 & 0 \\
& $\mathbf{1 0}$ & 0.093 & 0.084 & 0.004 & 0.011 \\
& $\mathbf{2 0}$ & 0.176 & 0.159 & 0.088 & 0.066 \\
& $\mathbf{5 0}$ & 0.333 & 0.318 & 0.263 & 0.230 \\
\hline
\end{tabular}

Table 8 


\begin{tabular}{|c|c|clll|}
\hline \multicolumn{7}{|c|}{ POG score for Leukimia } \\
\hline Method & subset/length & $\mathbf{5 0}$ & $\mathbf{1 0 0}$ & $\mathbf{5 0 0}$ & $\mathbf{1 0 0 0}$ \\
\hline T-test & $\mathbf{6}$ & 0.177 & 0.204 & 0.340 & 0.485 \\
& $\mathbf{1 0}$ & 0.280 & 0.340 & 0.456 & 0.547 \\
& $\mathbf{2 0}$ & 0.582 & 0.591 & 0.662 & 0.718 \\
& $\mathbf{5 0}$ & 0.750 & 0.784 & 0.817 & 0.844 \\
\hline Empirical Bayes & $\mathbf{6}$ & 0.246 & 0.272 & 0.378 & 0.497 \\
& $\mathbf{1 0}$ & 0.328 & 0.378 & 0.470 & 0.552 \\
& $\mathbf{2 0}$ & 0.602 & 0.608 & 0.667 & 0.721 \\
& $\mathbf{5 0}$ & 0.759 & 0.791 & 0.818 & 0.845 \\
\hline SAM & $\mathbf{6}$ & 0.266 & 0.294 & 0.408 & 0.511 \\
& $\mathbf{1 0}$ & 0.366 & 0.404 & 0.487 & 0.556 \\
& $\mathbf{2 0}$ & 0.657 & 0.672 & 0.698 & 0.729 \\
& $\mathbf{5 0}$ & 0.823 & 0.833 & 0.839 & 0.850 \\
\hline
\end{tabular}

Table 9

\begin{tabular}{|c|c|clll|}
\hline \multicolumn{7}{|c|}{ POGR score for Leukimia } \\
\hline Method & subset/length & $\mathbf{5 0}$ & $\mathbf{1 0 0}$ & $\mathbf{5 0 0}$ & $\mathbf{1 0 0 0}$ \\
\hline T-test & $\mathbf{6}$ & 0.549 & 0.552 & 0.650 & 0.737 \\
& $\mathbf{1 0}$ & 0.657 & 0.684 & 0.712 & 0.768 \\
& $\mathbf{2 0}$ & 0.834 & 0.816 & 0.821 & 0.845 \\
& $\mathbf{5 0}$ & 0.917 & 0.909 & 0.906 & 0.921 \\
\hline Empirical Bayes & $\mathbf{6}$ & 0.632 & 0.616 & 0.677 & 0.746 \\
& $\mathbf{1 0}$ & 0.706 & 0.711 & 0.720 & 0.771 \\
& $\mathbf{2 0}$ & 0.843 & 0.836 & 0.824 & 0.846 \\
& $\mathbf{5 0}$ & 0.921 & 0.917 & 0.906 & 0.921 \\
\hline SAM & $\mathbf{6}$ & 0.623 & 0.652 & 0.706 & 0.761 \\
& $\mathbf{1 0}$ & 0.708 & 0.749 & 0.746 & 0.783 \\
& $\mathbf{2 0}$ & 0.831 & 0.896 & 0.853 & 0.856 \\
& $\mathbf{5 0}$ & 0.914 & 0.954 & 0.927 & 0.927 \\
\hline
\end{tabular}

Table 10 


\begin{tabular}{|c|c|clll|}
\hline \multicolumn{7}{|c|}{ nPOG score for Leukimia } \\
\hline Method & subset/length & $\mathbf{5 0}$ & $\mathbf{1 0 0}$ & $\mathbf{5 0 0}$ & $\mathbf{1 0 0 0}$ \\
\hline T-test & $\mathbf{6}$ & 0.160 & 0.172 & 0.190 & 0.182 \\
& $\mathbf{1 0}$ & 0.265 & 0.312 & 0.330 & 0.281 \\
& $\mathbf{2 0}$ & 0.574 & 0.576 & 0.585 & 0.552 \\
& $\mathbf{5 0}$ & 0.745 & 0.776 & 0.775 & 0.753 \\
\hline Empirical Bayes & $\mathbf{6}$ & 0.231 & 0.242 & 0.237 & 0.202 \\
& $\mathbf{1 0}$ & 0.314 & 0.352 & 0.348 & 0.289 \\
& $\mathbf{2 0}$ & 0.593 & 0.593 & 0.592 & 0.556 \\
& $\mathbf{5 0}$ & 0.755 & 0.783 & 0.777 & 0.755 \\
\hline SAM & $\mathbf{6}$ & 0.250 & 0.266 & 0.273 & 0.223 \\
& $\mathbf{1 0}$ & 0.353 & 0.380 & 0.369 & 0.295 \\
& $\mathbf{2 0}$ & 0.650 & 0.659 & 0.629 & 0.568 \\
& $\mathbf{5 0}$ & 0.820 & 0.826 & 0.803 & 0.762 \\
\hline
\end{tabular}

Table 11

\begin{tabular}{|c|c|cccc|}
\hline \multicolumn{7}{|c|}{ nPOGR score for Leukimia } \\
\hline Method & subset/length & $\mathbf{5 0}$ & $\mathbf{1 0 0}$ & $\mathbf{5 0 0}$ & $\mathbf{1 0 0 0}$ \\
\hline T-test & $\mathbf{6}$ & 0.486 & 0.441 & 0.352 & 0.287 \\
& $\mathbf{1 0}$ & 0.612 & 0.610 & 0.466 & 0.372 \\
& $\mathbf{2 0}$ & 0.812 & 0.773 & 0.667 & 0.579 \\
& $\mathbf{5 0}$ & 0.906 & 0.886 & 0.825 & 0.786 \\
\hline Empirical Bayes & $\mathbf{6}$ & 0.581 & 0.522 & 0.403 & 0.313 \\
& $\mathbf{1 0}$ & 0.668 & 0.644 & 0.481 & 0.380 \\
& $\mathbf{2 0}$ & 0.823 & 0.797 & 0.672 & 0.581 \\
& $\mathbf{5 0}$ & 0.910 & 0.896 & 0.825 & 0.785 \\
\hline SAM & $\mathbf{6}$ & 0.571 & 0.567 & 0.455 & 0.354 \\
& $\mathbf{1 0}$ & 0.670 & 0.690 & 0.530 & 0.412 \\
& $\mathbf{2 0}$ & 0.809 & 0.871 & 0.727 & 0.608 \\
& $\mathbf{5 0}$ & 0.902 & 0.943 & 0.863 & 0.803 \\
\hline
\end{tabular}

Table 12 


\begin{tabular}{|c|c|lllc|}
\hline \multicolumn{7}{|c|}{ nPOG score for lung Cancer } \\
\hline Method & subset/length & $\mathbf{5 0}$ & $\mathbf{1 0 0}$ & $\mathbf{5 0 0}$ & $\mathbf{1 0 0 0}$ \\
\hline T-test & $\mathbf{4}$ & 0.083 & 0.110 & 0.204 & 0.179 \\
& $\mathbf{8}$ & 0.094 & 0.122 & 0.201 & 0.190 \\
& $\mathbf{1 2}$ & 0.112 & 0.159 & 0.266 & 0.242 \\
& $\mathbf{1 6}$ & 0.130 & 0.193 & 0.303 & 0.271 \\
\hline Empirical Bayes & $\mathbf{4}$ & 0.166 & 0.209 & 0.255 & 0.191 \\
& $\mathbf{8}$ & 0.167 & 0.209 & 0.256 & 0.201 \\
& $\mathbf{1 2}$ & 0.177 & 0.231 & 0.299 & 0.251 \\
& $\mathbf{1 6}$ & 0.188 & 0.248 & 0.321 & 0.284 \\
\hline SAM & $\mathbf{4}$ & 0.166 & 0.218 & 0.261 & 0.194 \\
& $\mathbf{8}$ & 0.166 & 0.221 & 0.263 & 0.204 \\
& $\mathbf{1 2}$ & 0.183 & 0.247 & 0.308 & 0.254 \\
& $\mathbf{1 6}$ & 0.202 & 0.270 & 0.330 & 0.290 \\
\hline
\end{tabular}

Table 13

\begin{tabular}{|c|c|c|c|c|c|c|c|c|c|}
\hline \multicolumn{10}{|c|}{ nPOGR 0.75 score for Lung Cancer } \\
\hline \multirow[b]{2}{*}{ Method } & \multirow[b]{2}{*}{ subset/length } & \multicolumn{4}{|c|}{ From cDNA to Affymetrix } & \multicolumn{4}{|c|}{ From Affymetrix to cDNA } \\
\hline & & 50 & 100 & 500 & 1000 & 50 & 100 & 500 & 1000 \\
\hline \multirow[t]{4}{*}{ T-test } & 4 & 0.172 & 0.041 & 0.033 & 0.009 & 0.191 & 0.081 & 0.042 & 0.010 \\
\hline & 8 & 0.212 & 0.054 & 0.047 & 0.012 & 0.232 & 0.094 & 0.050 & 0.019 \\
\hline & 12 & 0.311 & 0.153 & 0.058 & 0.034 & 0.321 & 0.173 & 0.058 & 0.041 \\
\hline & 16 & 0.377 & 0.214 & 0.107 & 0.086 & 0.365 & 0.218 & 0.107 & 0.092 \\
\hline \multirow{4}{*}{$\begin{array}{c}\text { Empirical } \\
\text { Bayes }\end{array}$} & 4 & 0.465 & 0.416 & 0.267 & 0.178 & 0.342 & 0.340 & 0.272 & 0.190 \\
\hline & 8 & 0.472 & 0.429 & 0.265 & 0.188 & 0.354 & 0.353 & 0.272 & 0.198 \\
\hline & 12 & 0.512 & 0.457 & 0.304 & 0.237 & 0.406 & 0.386 & 0.318 & 0.248 \\
\hline & 16 & 0.550 & 0.477 & 0.325 & 0.271 & 0.401 & 0.409 & 0.340 & 0.283 \\
\hline \multirow[t]{4}{*}{ SAM } & 4 & 0.468 & 0.436 & 0.272 & 0.181 & 0.345 & 0.353 & 0.278 & 0.191 \\
\hline & 8 & 0.466 & 0.435 & 0.271 & 0.190 & 0.361 & 0.367 & 0.278 & 0.200 \\
\hline & 12 & 0.519 & 0.475 & 0.312 & 0.240 & 0.396 & 0.388 & 0.325 & 0.250 \\
\hline & 16 & 0.538 & 0.487 & 0.332 & 0.277 & 0.389 & 0.412 & 0.348 & 0.288 \\
\hline
\end{tabular}

Table 14 


\begin{tabular}{|c|c|cccc|}
\hline \multicolumn{7}{|c|}{ nPOG score for DMD } \\
\hline Method & subset/length & $\mathbf{5 0}$ & $\mathbf{1 0 0}$ & $\mathbf{5 0 0}$ & $\mathbf{1 0 0 0}$ \\
\hline T-test & $\mathbf{6}$ & 0.061 & 0.085 & 0.163 & 0.165 \\
& $\mathbf{1 2}$ & 0.190 & 0.246 & 0.327 & 0.296 \\
& $\mathbf{1 8}$ & 0.256 & 0.333 & 0.404 & 0.376 \\
& $\mathbf{2 4}$ & 0.296 & 0.429 & 0.455 & 0.422 \\
\hline Empirical Bayes & $\mathbf{6}$ & 0.242 & 0.261 & 0.278 & 0.221 \\
& $\mathbf{1 2}$ & 0.297 & 0.348 & 0.375 & 0.319 \\
& $\mathbf{1 8}$ & 0.319 & 0.410 & 0.432 & 0.394 \\
& $\mathbf{2 4}$ & 0.377 & 0.496 & 0.485 & 0.431 \\
\hline SAM & $\mathbf{6}$ & 0.233 & 0.247 & 0.265 & 0.214 \\
& $\mathbf{1 2}$ & 0.306 & 0.346 & 0.372 & 0.318 \\
& $\mathbf{1 8}$ & 0.336 & 0.418 & 0.432 & 0.395 \\
& $\mathbf{2 4}$ & 0.383 & 0.492 & $\mathbf{0 . 4 8 5}$ & 0.436 \\
\hline
\end{tabular}

Table 15

\begin{tabular}{|c|c|c|c|c|c|c|c|c|c|}
\hline \multicolumn{10}{|c|}{ nPOGR 0.75 score for DMD Cancer } \\
\hline \multirow[b]{2}{*}{ Method } & \multirow[b]{2}{*}{ subset/length } & \multicolumn{4}{|c|}{ From Haslett to Pescatori } & \multicolumn{4}{|c|}{ From Pescatori to Haslett } \\
\hline & & $\mathbf{5 0}$ & 100 & 500 & 1000 & 50 & 100 & 500 & 1000 \\
\hline \multirow[t]{4}{*}{ T-test } & 6 & 0.178 & 0.169 & 0.147 & 0.138 & 0.185 & 0.174 & 0.141 & 0.138 \\
\hline & 12 & 0.247 & 0.232 & 0.143 & 0.117 & 0.263 & 0.222 & 0.163 & 0.117 \\
\hline & 18 & 0.383 & 0.188 & 0181 & 0.099 & 0.397 & 0.268 & 0.221 & 0.129 \\
\hline & 24 & 0.377 & 0.299 & 0.216 & 0.159 & 0.407 & 0.312 & 0.245 & 0.174 \\
\hline \multirow{4}{*}{$\begin{array}{c}\text { Empirical } \\
\text { Bayes }\end{array}$} & 6 & 0.601 & 0.598 & 0.422 & 0.267 & 0.636 & 0.604 & 0.423 & 0.276 \\
\hline & 12 & 0.688 & 0.678 & 0.469 & 0.344 & 0.736 & 0.691 & 0.487 & 0.344 \\
\hline & 18 & 0.714 & 0.711 & 0.504 & 0.409 & 0.783 & 0.740 & 0.531 & 0.399 \\
\hline & 24 & 0.725 & 0.749 & 0.530 & 0.437 & 0.803 & 0.775 & 0.570 & 0.435 \\
\hline \multirow[t]{4}{*}{ SAM } & 6 & 0.591 & 0.586 & 0.406 & 0.261 & 0.614 & 0.582 & 0.405 & 0.262 \\
\hline & 12 & 0.687 & 0.674 & 0.466 & 0.345 & 0.730 & 0.681 & 0.479 & 0.340 \\
\hline & 18 & 0.717 & 0.707 & 0.506 & 0.409 & 0.774 & 0.743 & 0.530 & 0.402 \\
\hline & 24 & 0.697 & 0.747 & 0.529 & 0.440 & 0.812 & 0.793 & 0.571 & 0.442 \\
\hline
\end{tabular}

Table 16 


\section{APPENDIX B}

\section{$R$ code for 3.1}

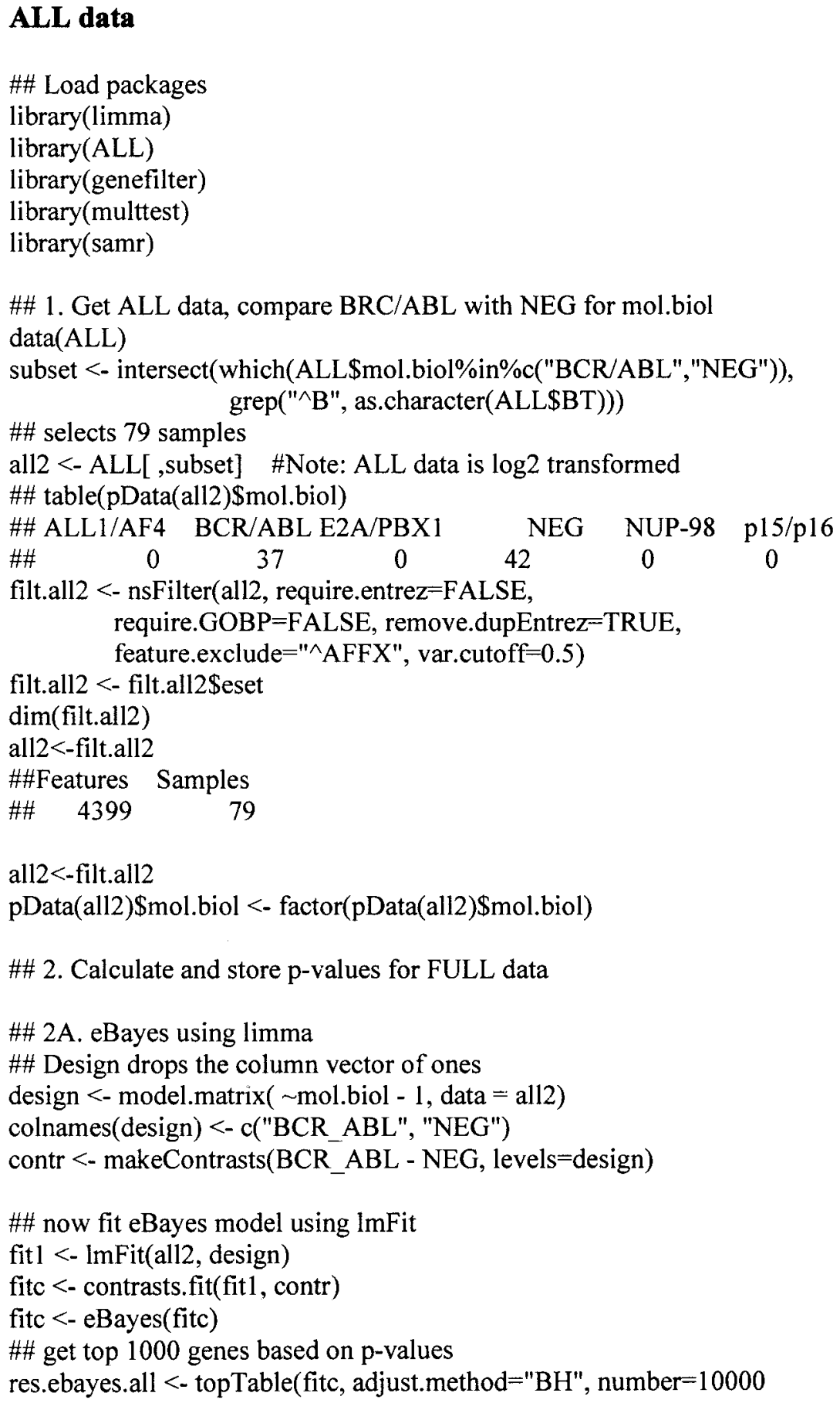




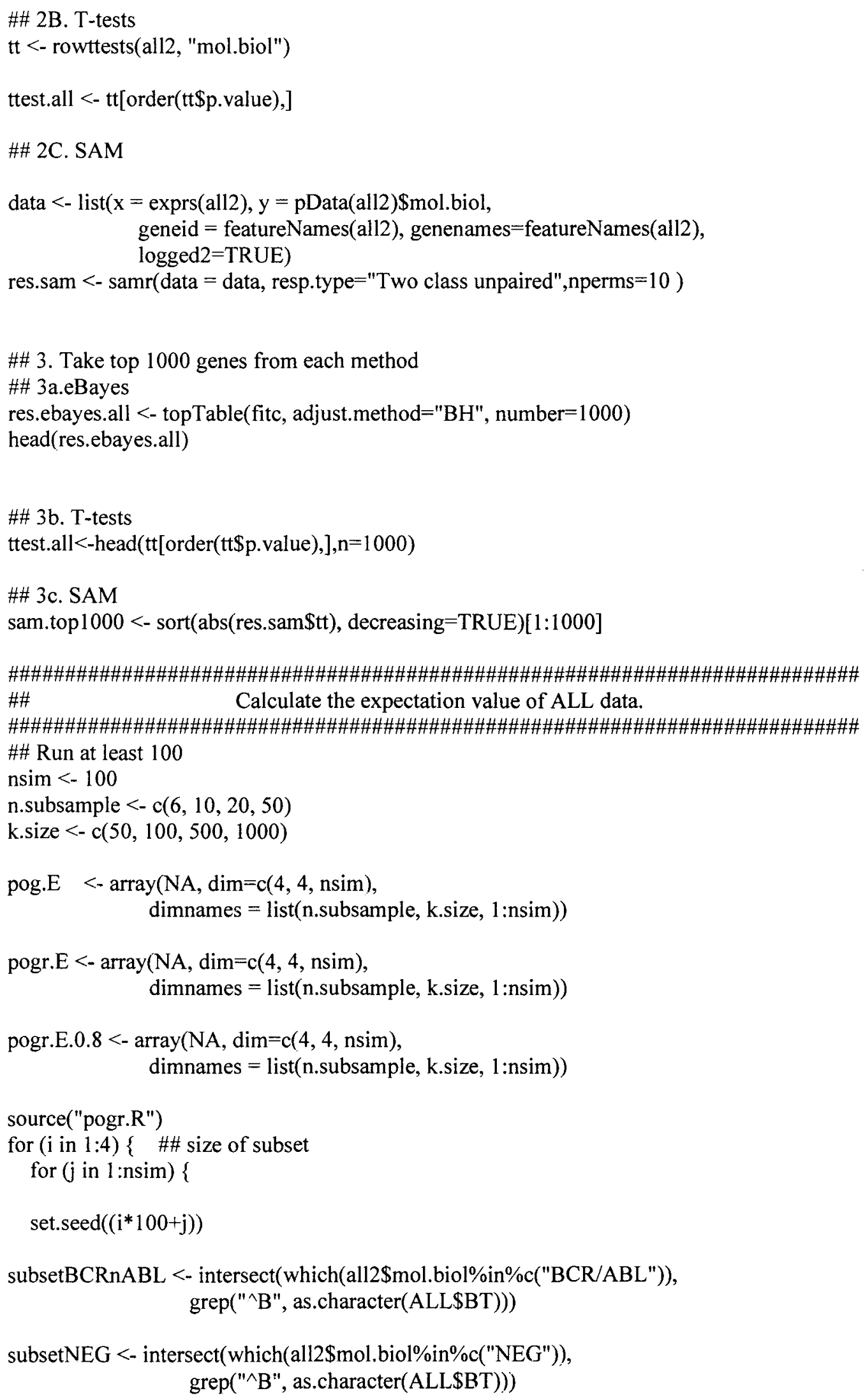


\#\# For subsets 1

sub.idxBCRnABL1 <- sample(subsetBCRnABL, n.subsample[i]/2, replace=FALSE)

sub.idxNEG1 <- sample(subsetNEG, n.subsample[i]/2, replace=FALSE)

sub.idx1<- union(sub.idxBCRnABL1,sub.idxNEG1 )

sub.mol.biol1 <- pData(all2)\$mol.biol[sub.idx1]

allsub1 $<-$ all2[,sub.idx1]

\#\# 1. Randomly pick two lists (size 1000)

\#\# head(exprs(allsub1))

idx1 <- sample(1:nrow(allsub1), 1000, replace=FALSE)

idx $2<$ - sample( 1 :nrow(all2), 1000, replace=FALSE)

list $1<$ - rownames(exprs(allsub1))[idx1]

list $2<$ - rownames(exprs(all2))[idx2]

\#\# Check overlap with original gene list in terms of ranking for top 1000

\#\# First, simple E[POG] score

pog.E[i,4, j] <- length(intersect(list $1[1: 1000]$, list2[1:1000]))/1000

pog.E[i,3, j] <- length(intersect(list1 [1:500], list2[1:500]))/500

pog.E[i,2, j] <- length(intersect(list $1[1: 100]$, list $2[1: 100])) / 100$

pog.E[i,1, j] <- length(intersect(list $1[1: 50]$, list $2[1: 50])) / 50$

all.genes $<-$ union(list1, list2)

cor.all.genes $<-\operatorname{cor}(t(\operatorname{exprs}($ all2 $)[$ all.genes, ]))

$\operatorname{diag}$ (cor.all.genes) $<-0$

pogr.E[i, 4, j] <- pogr(cor.all.genes, list1[1:1000], list2[1:1000], 0.5)

pogr.E[i, 3, j] <- pogr(cor.all.genes, list1[1:500], list2[1:500], 0.5)

pogr.E[i, 2, j] <- pogr(cor.all.genes, list1[1:100], list2[1:100], 0.5)

pogr.E[i, 1, j] <- pogr(cor.all.genes, list1[1:50], list2[1:50], 0.5)

pogr.E.0.8[i, 4, j] <- pogr(cor.all.genes, list1[1:1000], list2[1:1000], 0.8)

pogr.E.0.8[i, 3,j] <- pogr(cor.all.genes, list 1 [1:500], list2[1:500], 0.8)

pogr.E.0.8[i, 2, j] <- pogr(cor.all.genes, list1 [1:100], list2[1:100], 0.8)

pogr.E.0.8[i, 1, j] <- pogr(cor.all.genes, list1 [1:50], list2[1:50], 0.8)

3\}

\#\# cutoff $=0.5$

exp.POG <- ftable(apply(pog.E, $c(1,2)$, mean)) \#\# saved as a matrix

exp.POGR <- ftable(apply(pogr.E, c $(1,2)$, mean))

\#\# cutoff $=0.8$

exp.POGR.0.8 <- ftable(apply(pogr.E.0.8, c(1,2), mean))

\#\#write.csv( )

write.csv(exp.POG,file="E(pog).csv",row.names =FALSE)

write.csv(exp.POGR,file="E(pogr).csv",row.names =FALSE)

\#\# cutoff $=0.8$

write.csv(exp.POGR.0.8,file="E(pogr)08.csv",row.names =FALSE) 


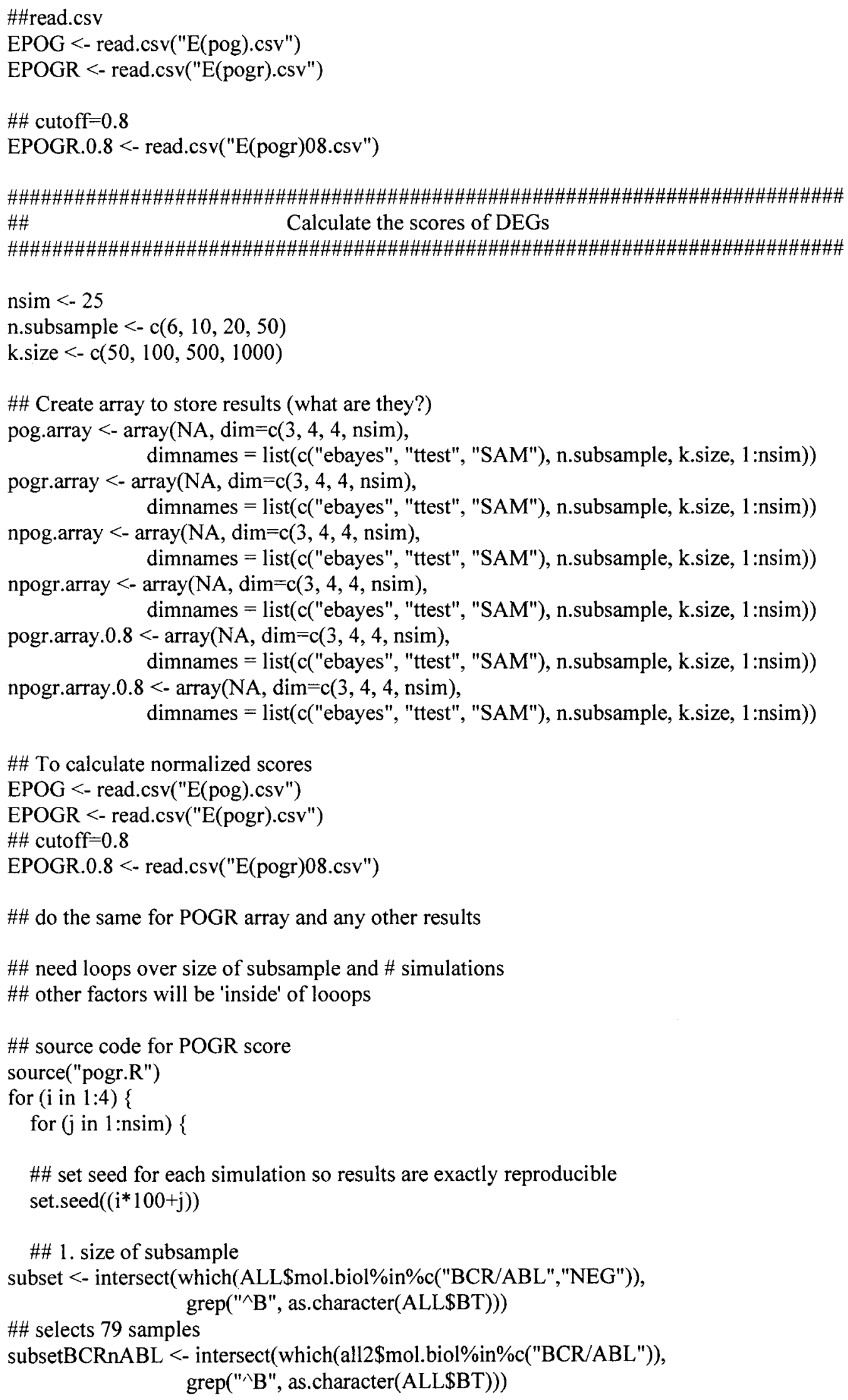


subsetNEG <- intersect(which(all2\$mol.biol\%in\%c("NEG")), grep("^B", as.character(ALL\$BT)))

sub.idxBCRnABL <- sample(subsetBCRnABL, n.subsample[i]/2, replace=FALSE)

sub.idxNEG <- sample(subsetNEG, n.subsample[i]/2, replace=FALSE)

sub.idx<- union(sub.idxBCRnABL,sub.idxNEG)

sub.mol.biol <- pData(all2)\$mol.biol[sub.idx]

allsub $<$ - all2[,sub.idx]

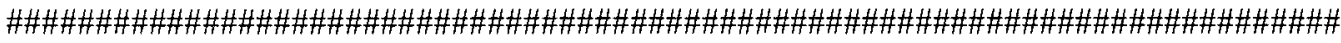

\#\#

2A. E-BAYES (LIMMA) METHOD

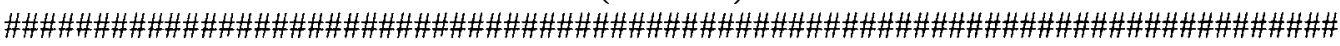

lnames(design) <- c("BCR_ABL", "NEG")

contr $<-$ makeContrasts $\left(B \overline{C R} \_A B L-N E G\right.$, levels=design)

fitl sub $<-$ lmFit(all2 [,sub.idx], design)

fitcsub $<$ - contrasts.fit(fit 1 sub, contr)

fitcsub <- eBayes(fitcsub)

\#\# Full gene list

res.ebayes.all <- topTable(fitc, adjust.method="BH", number=1000)

ressub $<-$ topTable(fitcsub, adjust.method="BH", number=1000)

pog.array["ebayes",i, 4, j] <- length(intersect(ressub\$ID[1:1000],

res.ebayes.all\$ID[1:1000]))/1000

pog.array["ebayes",i, 3, j] <- length(intersect(ressub\$ID[1:500],

res.ebayes.all\$ID[1:500]))/500

pog.array["ebayes",i, 2, j] <- length(intersect(ressub\$ID[1:100],

res.ebayes.all\$ID $[1: 100])) / 100$

pog.array["ebayes",i, $1, \mathrm{j}]<-$ length(intersect(ressub\$ID[1:50], res.ebayes.all $\$ \operatorname{ID}[1: 50])) / 50$

\#\# Normalized POG

npog.array["ebayes",i, 4, j] <- (pog.array["ebayes",i, 4, j] - EPOG[i,4])/(1-EPOG[i,4])

npog.array["ebayes",i, 3, j] <- (pog.array["ebayes",i, 3, j] - EPOG[i,3])/(1-EPOG[i,3])

npog.array["ebayes",i, 2, j] <- (pog.array["ebayes",i, 2, j] - EPOG[i,2])/(1-EPOG[i,2])

npog.array["ebayes",i, 1, j] <- (pog.array["ebayes",i, 1, j] - EPOG[i,1])/(1-EPOG[i,1])

\#\# Now, look at POGR score

all.genes $<-$ union(ressub\$ID, res ebayes.all\$ID)

cor.all.genes $<-$ cor(t(exprs(all2)[all.genes, ]))

diag(cor.all.genes) $<-0$

\#\# cutoff $=0.5$

pogr.array["ebayes",i, 4, j] <- pogr(cor.all.genes, res.ebayes.all\$ID[1:1000],

ressub\$ID[1:1000], 0.5)

pogr.array["ebayes",i, 3, j] <- pogr(cor.all.genes, res.ebayes.all\$ID[1:500], ressub\$ID[1:500], $0.5)$

pogr.array["ebayes",i, 2, j] <- pogr(cor.all.genes, res.ebayes.all\$ID[1:100], ressub\$ID[1:100], $0.5)$

pogr.array["ebayes",i, 1, j] <- pogr(cor.all.genes, res.ebayes.all\$ID[1:50], ressub\$ID[1:50], $0.5)$

npogr.array["ebayes",i, 4, j] <- (pogr.array["ebayes",i, 4, j] - EPOGR[i,4])/(1-EPOGR[i,4]) npogr.array["ebayes",i, 3, j] <- (pogr.array["ebayes",i, 3, j] - EPOGR[i,3])/(1-EPOGR[i,3]) npogr.array["ebayes",i, 2, j] <- (pogr.array["ebayes",i, 2,j] - EPOGR $[\mathrm{i}, 2]) /(1-\operatorname{EPOGR}[\mathrm{i}, 2])$ npogr.array["ebayes",i, 1, j] <- (pogr.array["ebayes",i, 1,j] - EPOGR[i,1])/(1-EPOGR[i,1]) 
\#\# cutoff $=0.8$

pogr.array. $0.8[$ "ebayes", $\mathrm{i}, 4, \mathrm{j}]<-$ pogr(cor.all.genes, res.ebayes.all\$ID[1:1000],

ressub\$ID $[1: 1000], 0.8)$

pogr.array.0.8["ebayes",i, 3, j] <- pogr(cor.all.genes, res.ebayes.all\$ID[1:500], ressub\$ID $[1: 500], 0.8)$

pogr.array.0.8["ebayes",i, 2, j] <- pogr(cor.all.genes, res.ebayes.all\$ID[1:100], ressub\$ID[1:100], 0.8)

pogr.array.0.8["ebayes",i, $1, \mathrm{j}]<-$ pogr(cor.all.genes, res.ebayes.all\$ID[1:50], ressub\$ID[1:50], $0.8)$

npogr.array. $0.8[$ "ebayes",i, $4, \mathrm{j}]<-$ (pogr.array.0.8["ebayes",i, 4, j] EPOGR.0.8[i,4])/(1-EPOGR.0.8[i,4])

npogr.array. $0.8[$ "ebayes",i, $3, \mathrm{j}]<-($ pogr.array. $0.8[$ "ebayes",i, 3, j] EPOGR.0.8[i,3])/(1-EPOGR.0.8[i,3])

npogr.array.0.8["ebayes",i, 2, j] <- (pogr.array.0.8["ebayes",i, 2, j] EPOGR.0.8[i,2])/(1-EPOGR.0.8[i,2])

npogr.array.0.8["ebayes",i, 1, j] <- (pogr.array.0.8["ebayes",i, 1, j] EPOGR.0.8[i,1])/(1-EPOGR.0.8[i,1])

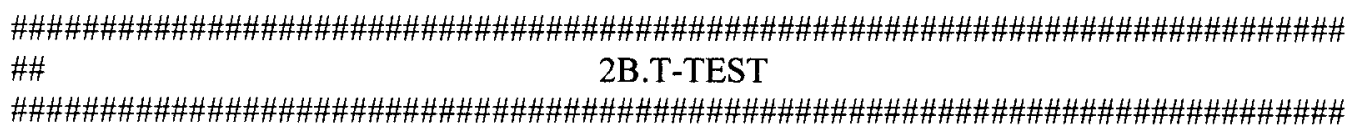

ttsub $<$ - rowttests(all2[,sub.idx], all2[,sub.idx]\$mol.biol)

ttest.sub $<$-head(ttsub[order(ttsub\$p.value),],n=1000)

ttest.all<-head(tt[order(tt\$p.value),],n=1000)

\#\# First, simple POG score

rownames(ttest.all)

rownames(ttest.sub)

pog.array["ttest",i, 4, j] <- length(intersect(rownames(ttest.all)[1:1000],

rownames(ttest.sub) $[1: 1000])) / 1000$

pog.array["ttest",i, 3, j] <- length(intersect(rownames(ttest.all)[1:500],

rownames(ttest.sub)[1:500]))/500

pog.array["ttest",i, 2, j] <- length(intersect(rownames(ttest.all)[1:100],

rownames(ttest.sub) $[1: 100])) / 100$

pog.array["ttest",i, 1, j] <- length(intersect(rownames(ttest.all)[1:50],

rownames(ttest.sub) $[1: 50])) / 50$

\#\# Normalized POG

npog.array["ttest",i, 4, j] <- (pog.array["ttest",i, 4, j] - EPOG[i,4])/(1-EPOG[i,4])

npog.array["ttest",i, 3, j] <- (pog.array["ttest",i, 3, j] - EPOG[i,3])/(1-EPOG[i,3])

npog.array["ttest",i, 2, j] <- (pog.array["ttest",i, 2, j] - EPOG[i,2])/(1-EPOG[i,2])

npog.array["ttest",i, 1, j] <- (pog.array["ttest",i, 1, j] - EPOG[i,1])/(1-EPOG[i,1])

\#\# Now, look at POGR score

all.genes $<-$ union(rownames(ttest.all), rownames(ttest.sub))

cor.all.genes $<-$ cor(t(exprs(all2)[all.genes, ]))

\#\# need to find which are significant (just pick a cut-off (say 0.5 ) and use that)

\#\# need to look at all OFF DIAGONAL values of cor.all.genes (set diagonal values to zero)

$\operatorname{diag}($ cor.all.genes $)<-0$ 
\#\# maybe have another dimension for correlation cutoff?? $(0.5,0.8)$

\#\# cutoff $=0.5$

pogr.array["ttest",i, 4, j] <- pogr(cor.all.genes, rownames(ttest.all)[1:1000], rownames(ttest.sub) $[1: 1000], 0.5)$

pogr.array["ttest",i, 3, j] <- pogr(cor.all.genes, rownames(ttest.all)[1:500], rownames(ttest.sub) [1:500], 0.5)

pogr.array["ttest",i, 2, j] <- pogr(cor.all.genes, rownames(ttest.all)[1:100], rownames(ttest.sub) [1:100], 0.5)

pogr.array["ttest",i, 1, j] <- pogr(cor.all.genes, rownames(ttest.all)[1:50], rownames(ttest.sub) $[1: 50], 0.5)$

npogr.array["ttest",i, 4, j] <- (pogr.array["ttest",i, 4, j] - EPOGR[i,4])/(1-EPOGR[i,4]) npogr.array["ttest",i, 3, j] <- (pogr.array["ttest",i, 3, j] - EPOGR[i,3])/(1-EPOGR[i,3]) npogr.array["ttest",i, 2, j] <- (pogr.array["ttest",i, 2, j] - EPOGR[i,2])/(1-EPOGR[i,2]) npogr.array["ttest",i, 1, j] <- (pogr.array["ttest",i, 1, j] - EPOGR[i,1])/(1-EPOGR[i,1])

\#\# cutoff $=0.8$

pogr.array. 0.8 ["ttest",i, $4, \mathrm{j}]<-$ pogr(cor.all.genes, rownames(ttest.all)[1:1000], rownames(ttest.sub) $[1: 1000], 0.8)$

pogr.array.0.8["ttest",i, 3, j] <- pogr(cor.all.genes, rownames(ttest.all)[1:500], rownames(ttest.sub) [1:500], 0.8)

pogr.array. $0.8[" t t e s t ", i, 2, j]<-\operatorname{pogr}($ cor.all.genes, rownames(ttest.all)[1:100], rownames(ttest.sub) $[1: 100], 0.8$ )

pogr.array.0.8["ttest",i, 1, j] <- pogr(cor.all.genes, rownames(ttest.all)[1:50], rownames(ttest.sub) $[1: 50], 0.8)$

npogr.array.0.8["ttest",i, $4, \mathrm{j}]<-$ (pogr.array.0.8["ttest",i, $4, \mathrm{j}]$ EPOGR.0.8[i,4])/(1-EPOGR.0.8[i,4])

npogr.array. $0.8[$ "ttest",i, 3, j] <- (pogr.array. $0.8[$ "ttest",i, 3, j] EPOGR.0.8[i,3])/(1-EPOGR.0.8[i,3])

npogr.array.0.8["ttest",i, 2, j] <- (pogr.array.0.8["ttest",i, 2, j] EPOGR.0.8[i,2])/(1-EPOGR.0.8[i,2])

npogr array.0.8["ttest",i, $1, \mathrm{j}]<-$ (pogr.array. $0.8[$ "ttest",i, 1, j] EPOGR.0.8[i,1])/(1-EPOGR.0.8[i,1])

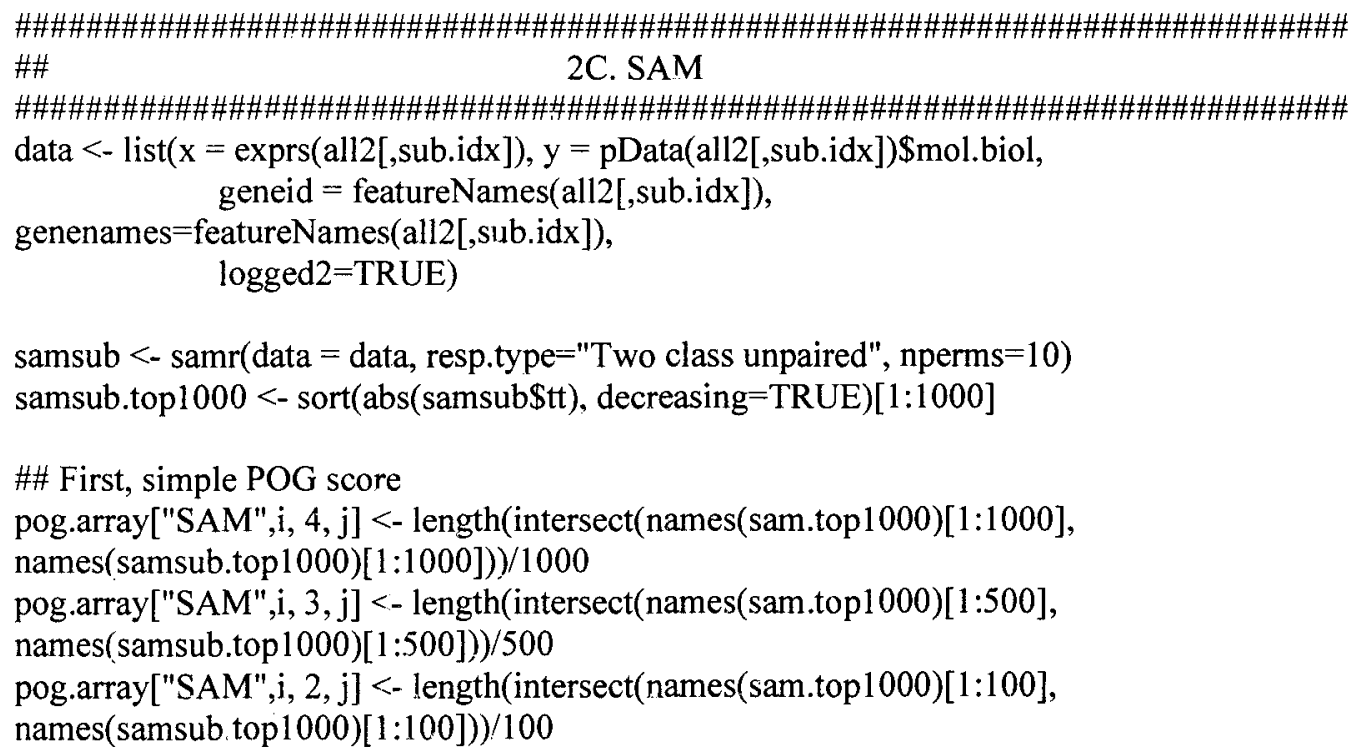


pog.array["SAM",i, 1, j] <- length(intersect(names(sam.top 1000)[1:50],

names(samsub.top 1000)[1:50]))/50

\#\# Normalized POG

npog.array["SAM",i, 4, j] <- (pog.array["SAM",i, 4, j] - EPOG[i,4])/(1-EPOG[i,4])

npog.array["SAM",i, 3, j] <- (pog.array["SAM",i, 3, j] - EPOG[i,3])/(1-EPOG[i,3])

npog.array["SAM",i, 2, j] <- (pog.array["SAM",i, 2, j] - EPOG[i,2])/(1-EPOG[i,2])

npog.array["SAM",i, 1, j] <- (pog.array["SAM",i, 1, j] - EPOG[i,1])/(1-EPOG[i,1])

\#\# Now, look at POGR score

all.genes <- union(names(samsub.top 1000), names(sam.top1000))

cor.all.genes $<-\operatorname{cor}(\mathrm{t}(\operatorname{exprs}($ all2 $)[$ all.genes, ]))

diag(cor.all.genes) $<-0$

\#\# cut point $=0.5$

pogr.array["SAM",i, 4, j] <- pogr(cor.all.genes, names(sam.top1000)[1:1000],

names(samsub.top 1000)[1:1000], 0.5)

pogr.array["SAM",i, 3, j] <- pogr(cor.all.genes, names(sam.top1000)[1:500],

names(samsub.top 1000)[1:500], 0.5)

pogr.array["SAM",i, 2, j] <- pogr(cor.all.genes, names(sam.top1000)[1:100],

names(samsub.top 1000)[1:100], 0.5)

pogr.array["SAM",i, 1, j] <- pogr(cor.all.genes, names(sam.top1000)[1:50],

names(samsub.top 1000)[1:50],0.5)

npogr.array["SAM",i, 4, j] <- (pogr.array["SAM",i, 4, j] - EPOGR[i,4])/(1-EPOGR[i,4])

npogr.array["SAM",i, 3, j] <- (pogr.array["SAM",i, 3, j] - EPOGR[i,3])/(1-EPOGR[i,3])

npogr.array["SAM",i, 2, j] <- (pogr.array["SAM",i, 2, j] - EPOGR[i,2])/(1-EPOGR[i,2])

npogr.array["SAM",i, 1,j] <- (pogr.array["SAM",i, 1, j] - EPOGR[i,1])/(1-EPOGR[i,1])

\#\# cutoff $=0.8$

pogr.array.0.8["SAM",i, 4, j] <- pogr(cor.all.genes, names(sam.top1000)[1:1000],

names(samsub.top 1000)[1:1000], 0.8)

pogr.array.0.8["SAM",i, 3, j] <- pogr(cor.all.genes, names(sam.top1000)[1:500],

names(samsub.top 1000)[1:500], 0.8)

pogr.array.0.8["SAM",i, 2, j]<- pogr(cor.all.genes, names(sam.top 1000)[1:100], names(samsub.top 1000)[1:100], 0.8)

pogr.array.0.8["SAM",i, 1, j] <- pogr(cor.all.genes, names(sam.top 1000)[1:50],

names(samsub.top 1000)[1:50], 0.8)

npogr.array.0.8["SAM",i, 4, j] <- (pogr.array.0.8["SAM",i, 4, j] -

EPOGR.0.8[i,4])/(1-EPOGR.0.8[i,4])

npogr.array.0.8["SAM",i, 3, j] <- (pogr.array.0.8["SAM",i, 3, j] -

EPOGR.0.8[i,3])/(1-EPOGR.0.8[i,3])

npogr.array.0.8["SAM",i, 2, j] <- (pogr.array.0.8["SAM",i, 2, j] -

EPOGR.0.8[i,2])/(1-EPOGR.0.8[i,2])

npogr.array.0.8["SAM",i, $1, \mathrm{j}]$ <- (pogr.array.0.8["SAM",i, 1, j] -

EPOGR.0.8[i,1])/(1-EPOGR.0.8[i,1])

\})

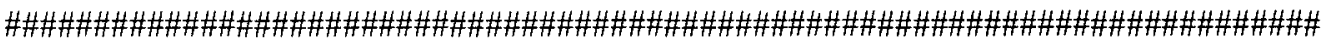

\#\# END OF SIMULATION LOOPS

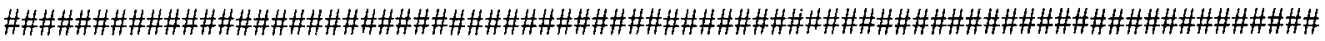


\#\# Codes for Conlon Cancer, Lung Expression and Leukimia are similar to the ALL data.

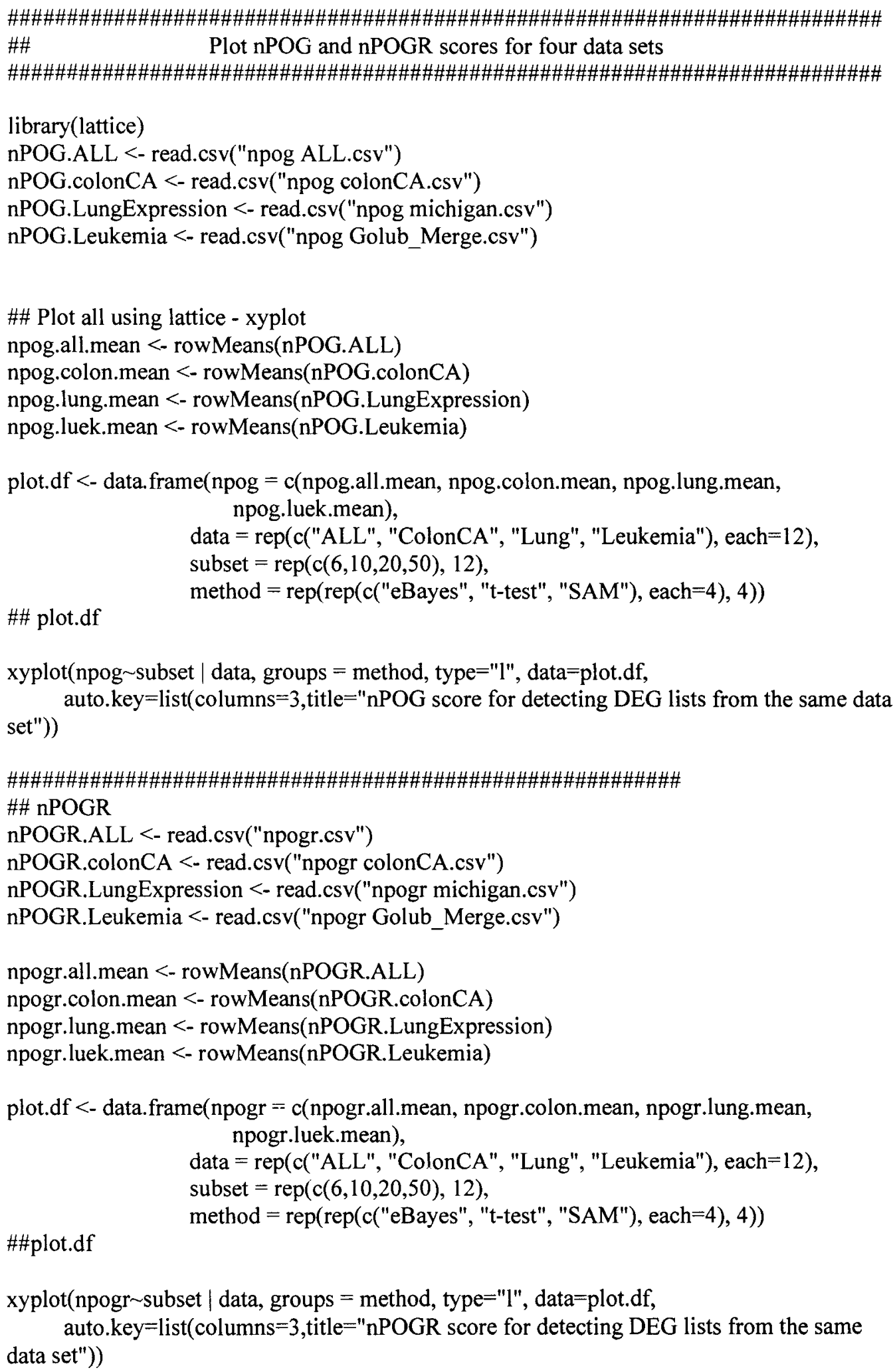




\section{$\mathbf{R}$ code for section 3.2}

\section{Prostate Cancer}

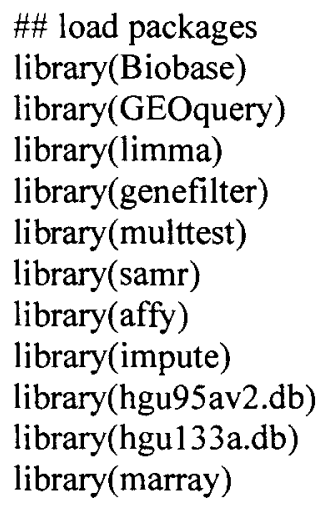
require.GOBP $=$ FALSE, remove.dupEntrez $=$ TRUE, 


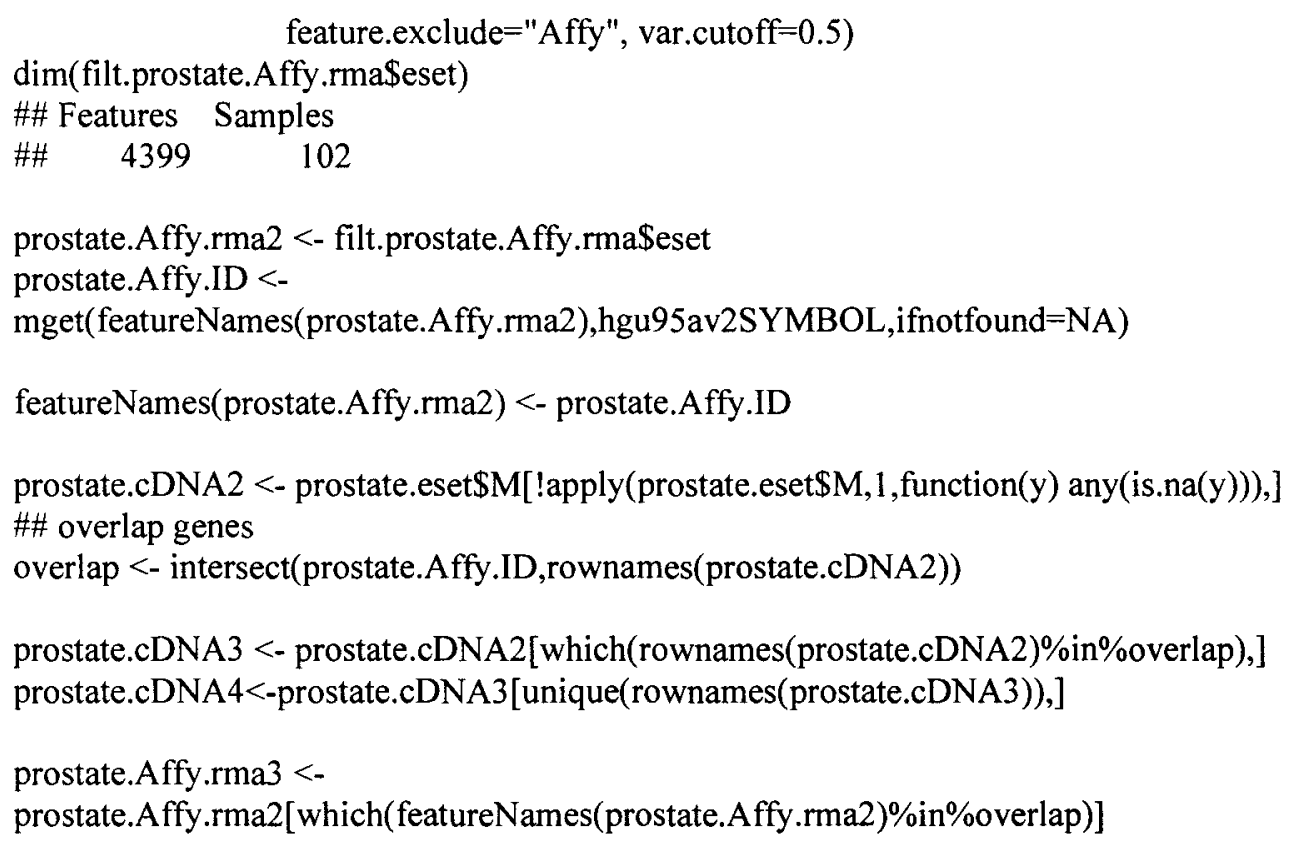


res.prostate.Affy <- topTable(fitc2, adjust.method="BH",number=1000)

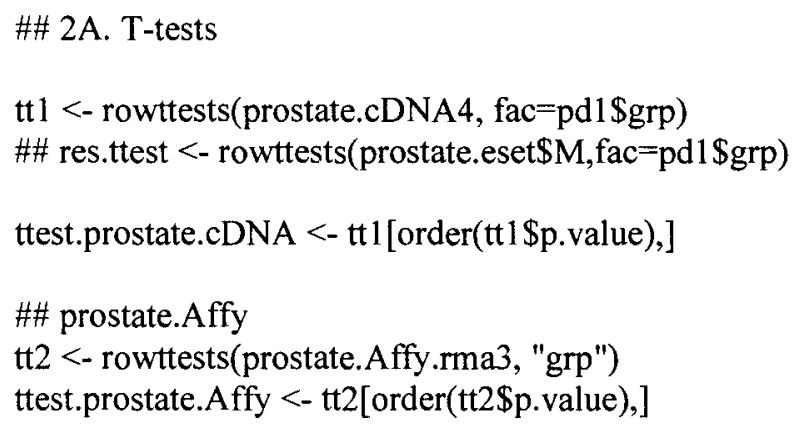


ebayes. prostate.Affy <- topTable(fitc2, adjust.method="BH", number=2000)

length(intersect(ebayes.prostate.cDNA\$ID,ebayes.prostate.Affy\$ID))

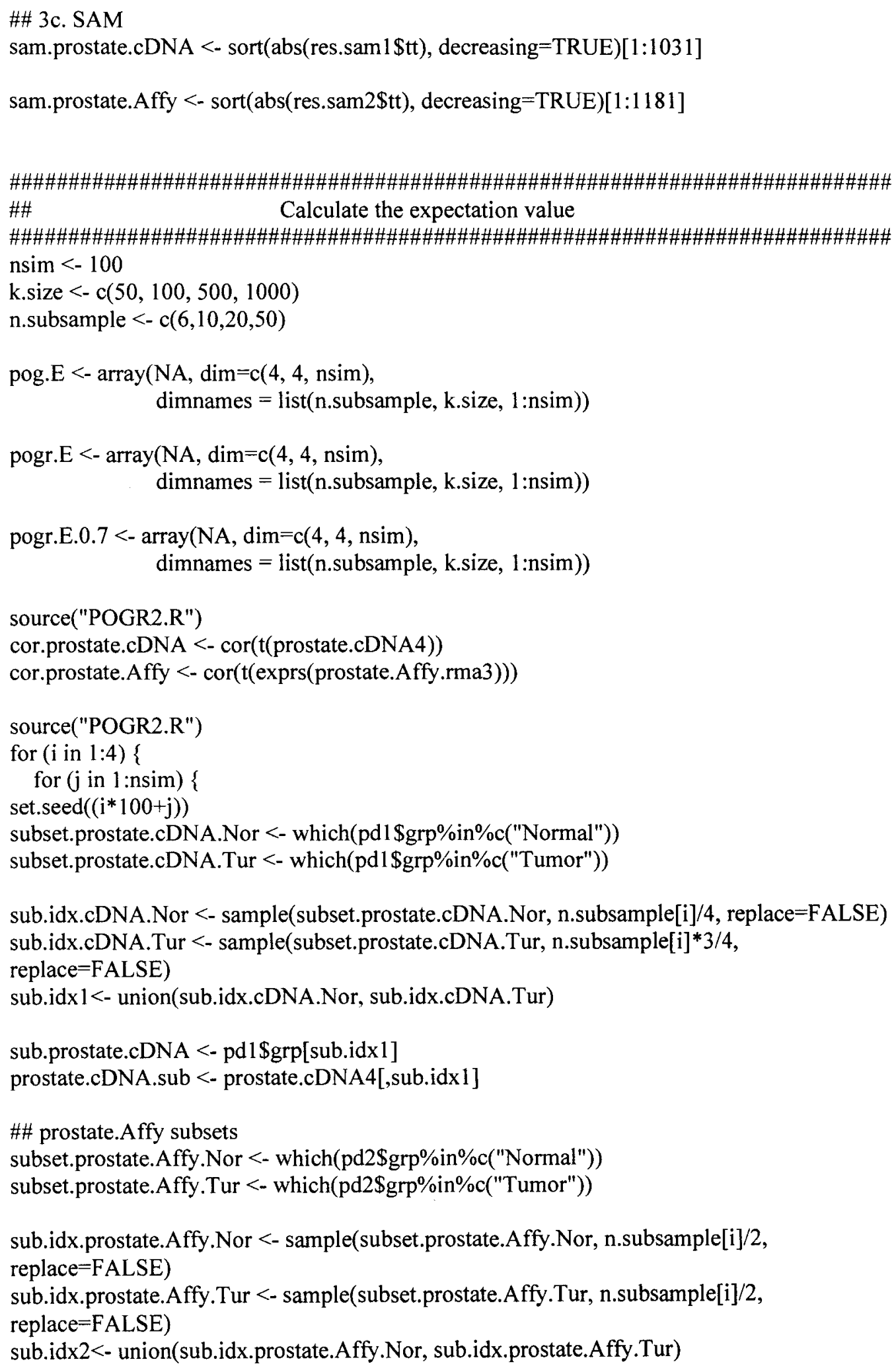


sub.prostate.Affy <- pData(prostate.Affy.rma3)[sub.idx2,]

prostate.Affy.sub <- prostate.Affy.rma3 [,sub.idx2]

\#\# 1. Randomly pick two lists (size 1000)

\#\# head(exprs(allsub1))

idx1 <- sample(1:nrow(prostate.cDNA.sub), 1000, replace=FALSE)

idx2 <- sample(1:nrow(prostate.Affy.sub ), 1000, replace=FALSE)

list $1<-$ rownames(prostate.cDNA.sub)[idxl]

list $2<-$ rownames(exprs(prostate.Affy.sub))[idx2]

\#\# Check overlap with original gene list in terms of ranking for top 1000

\#\# First, simple E[POG] score

pog.E[i, $4, \mathrm{j}]<-$ length(intersect(list $1[1: 1000]$, list2[1:1000]))/1000

pog.E[i, $3, \mathrm{j}]<-$ length(intersect(list $1[1: 500]$, list $2[1: 500])) / 500$

pog.E[i, $2, j]<-$ length(intersect(list $1[1: 100]$, list $2[1: 100])) / 100$

pog.E[i, $1, j]<-$ length(intersect(list 1[1:50], list2[1:50]))/50

\#\# Now, look at POGR.E score

\#\# Need to calculate all pairwise correlations between these two sets of genes

pogr.E[i, 4, j] <- pogr(cor.prostate.cDNA ,cor.prostate.Affy, list1[1:1000], list2[1:1000], 0.5)

pogr.E[i, 3, j] <- pogr(cor.prostate.cDNA ,cor.prostate.Affy, list1[1:500], list2[1:500], 0.5)

pogr.E[i, $2, \mathrm{j}]<-$ pogr(cor.prostate.cDNA ,cor.prostate.Affy, list1[1:100], list2[1:100], 0.5)

pogr.E[i, 1, j] <- pogr(cor.prostate.cDNA ,cor.prostate.Affy, list $1[1: 50]$, list2[1:50], 0.5)

pogr.E. $0.7[\mathrm{i}, 4, \mathrm{j}]<-$ pogr(cor.prostate.cDNA ,cor.prostate.Affy, list1[1:1000], list2[1:1000], $0.7)$

pogr.E.0.7[i, 3, j] <- pogr(cor.prostate.cDNA ,cor.prostate.Affy, list1[1:500], list2[1:500],

$0.7)$

pogr.E.0.7[i, 2, j] <- pogr(cor.prostate.cDNA ,cor.prostate.Affy, list1 [1:100], list2[1:100], $0.65)$

pogr.E. $0.7[\mathrm{i}, 1, \mathrm{j}]<-\operatorname{pogr}($ cor.prostate.cDNA ,cor.prostate.Affy, list1[1:50], list2[1:50], 0.7)

\})

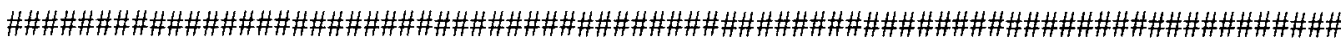

\#\# Calculate the scores

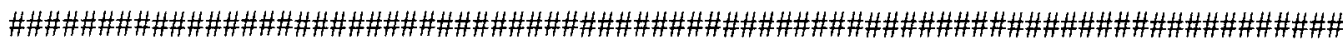

nsim $<-50$

k.size $<-c(50,100,500,1000)$

n.subsample $<-c(6,10,20,50)$

\#\# Create array to store results (what are they?)

pog.array $<-\operatorname{array}(\mathrm{NA}, \operatorname{dim}=\mathrm{c}(3,4,4, \mathrm{nsim})$, dimnames = list(c("ttest","ebayes", "SAM"),n.subsample, k.size, 1:nsim))

pogr.array $<-$ array $(\mathrm{NA}, \operatorname{dim}=\mathrm{c}(3,4,4, \mathrm{nsim})$, dimnames = list(c("ttest","ebayes", "SAM"),n.subsample, k.size, 1:nsim))

pogr.array $2<-\operatorname{array}(\mathrm{NA}, \operatorname{dim}=\mathrm{c}(3,4,4, \mathrm{nsim})$, dimnames = list(c("ttest","ebayes", "SAM"),n.subsample, k.size, 1 :nsim))

npog.array $<-$ array(NA, dim $=c(3,4,4, n \operatorname{nim})$, 


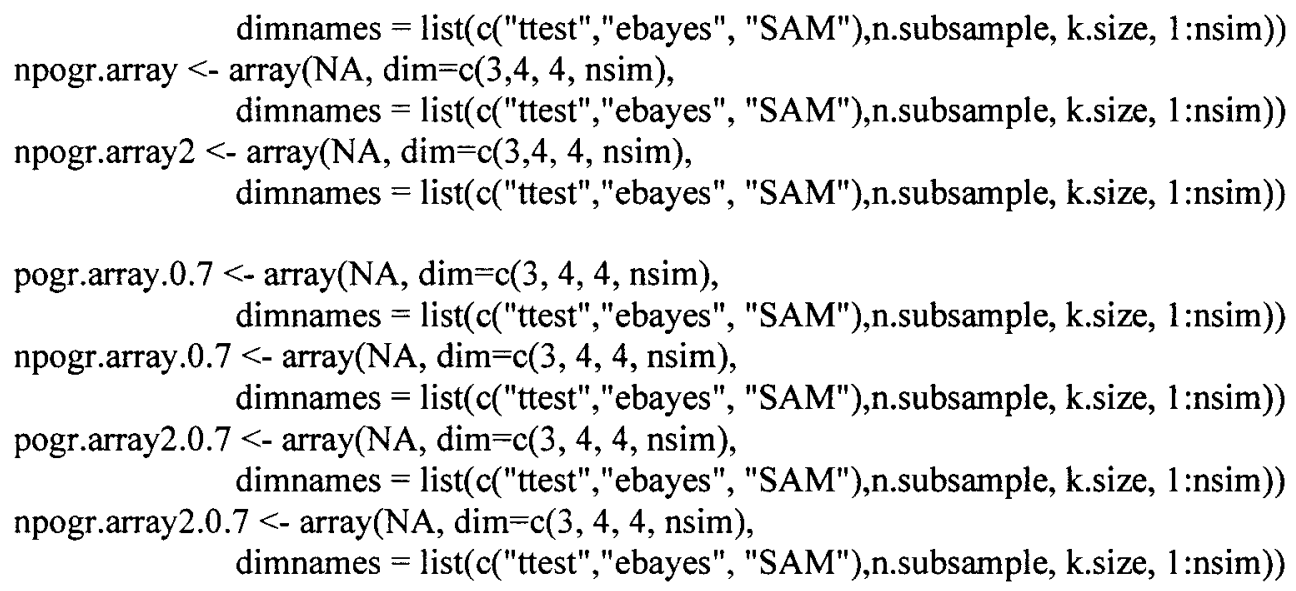

\#\# To calculate normalized scores

EPOG <- read.csv("E(pog)prostate.sub.csv")

EPOGR <- read.csv("E(pogr)prostate.sub.csv")

\#\# cutoff $=0.7$

EPOGR.0.7<- read.csv("E(pogr)prostate.sub.07.csv")

\#\# cor matrix for two datasets

cor.prostate.cDNA <- cor(t(prostate.cDNA4))

cor.prostate.Affy <- cor(t(exprs(prostate.Affy.rma3)))

source("POGR2.R")

for $(\mathrm{i}$ in $1: 4)\{$

for $(j$ in $1: n s i m)$ (

set.seed $\left(\left(i^{*} 100+j\right)\right)$

subset.prostate.cDNA.Nor <- which(pd1\$grp\%in\%c("Normal"))

subset.prostate.cDNA.Tur <- which(pd1\$grp\%in\%c("Tumor"))

sub.idx.cDNA.Nor <- sample(subset.prostate.cDNA.Nor, n.subsample[i]/2, replace=FALSE) sub.idx.cDNA.Tur <- sample(subset.prostate.cDNA.Tur, n.subsample[i]/2, replace=FALSE)

sub.idx $1<-$ union(sub.idx.cDNA.Nor, sub.idx.cDNA.Tur)

sub.prostate.cDNA <- pd1\$grp[sub.idx1]

prostate.cDNA.sub <- prostate.cDNA4[,sub.idx1]

\#\# prostate.Affy subsets

subset.prostate.Affy.Nor <- which(pd2\$grp\%in\%c("Normal"))

subset.prostate.Affy.Tur <- which(pd2\$grp\%in\%c("Tumor"))

sub.idx.prostate.Affy.Nor <- sample(subset.prostate.Affy.Nor, n.subsample[i]/2, replace $=$ FALSE)

sub.idx.prostate.Affy.Tur <- sample(subset.prostate.Affy.Tur, n.subsample[i]/2,

replace $=$ FALSE)

sub.idx2<- union(sub.idx.prostate.Affy.Nor, sub.idx.prostate.Affy.Tur)

sub.prostate.Affy <- pData(prostate.Affy.rma3)[sub.idx2,]

prostate.Affy.sub <- prostate.Affy.rma3 [,sub.idx2] 


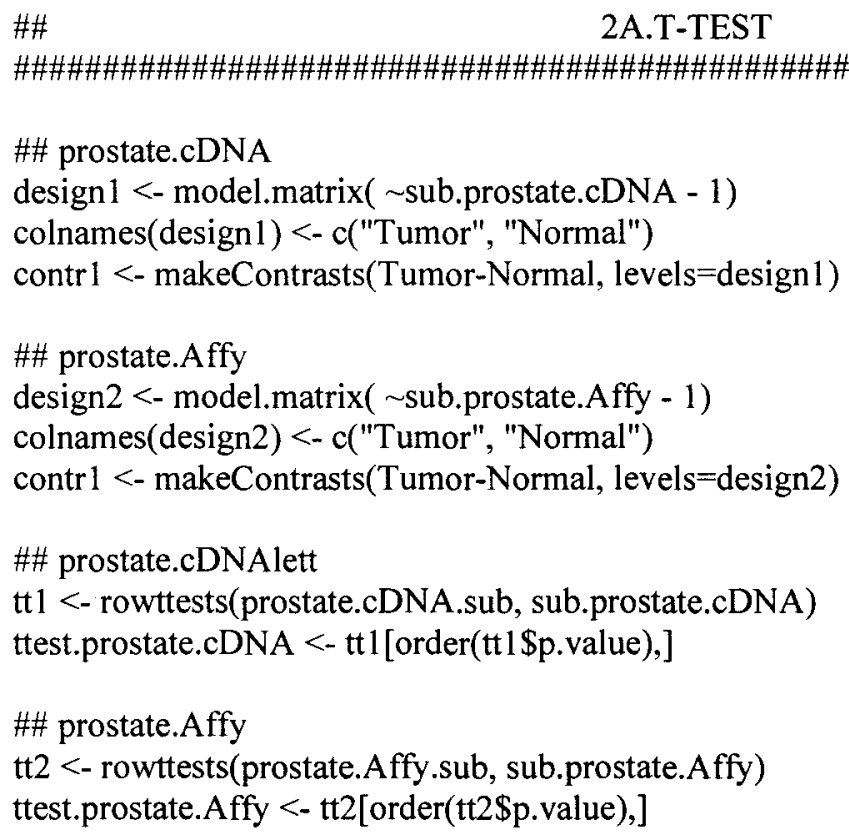

\#\# First, simple POG score

pog.array["ttest",i,4,j] <- length(intersect(rownames(ttest.prostate.cDNA)[1:1000], rownames(ttest.prostate.Affy) $[1: 1000])) / 1000$

pog.array["ttest",i,3,j]<- length(intersect(rownames(ttest.prostate.cDNA)[1:500], rownames(ttest.prostate.Affy) [1:500]))/500

pog.array["ttest",i,2,j] <- length(intersect(rownames(ttest.prostate.cDNA)[1:100], rownames(ttest.prostate.Affy) $[1: 100])) / 100$

pog.array["ttest",i,1,j] <- length(intersect(rownames(ttest.prostate.cDNA)[1:50], rownames(ttest.prostate.Affy) $[1: 50])) / 50$

\#\# Normalized POG npog.array["ttest",i,4,j] <- (pog.array["ttest",i,4,j] - EPOG[i,4])/(1-EPOG[i,4]) npog.array["ttest",i,3,j] <- (pog.array["ttest",i,3,j] - EPOG[i,3])/(1-EPOG[i,3]) npog.array["ttest",i,2,j] <- (pog.array["ttest",i,2,j] - EPOG[i,2])/(1-EPOG[i,2]) npog.array["ttest",i,1,j] <- (pog.array["ttest",i,1,j] - EPOG[i,1])/(1-EPOG[i,1])

\#\# Now, look at POGR score

\#\# cutoff $=0.5$

pogr.array["ttest",i,4,j] <- pogr(cor.prostate.cDNA,cor.prostate.Affy, rownames(ttest.prostate.cDNA) [1:1000],

rownames(ttest.prostate.Affy) $[1: 1000], 0.5)$

pogr.array["ttest",i,3,j] <- pogr(cor.prostate.cDNA,cor.prostate.Affy, rownames(ttest.prostate.cDNA)[1:500],

rownames(ttest.prostate.Affy) [1:500], 0.5)

pogr.array["ttest",i,2,j] <- pogr(cor.prostate.cDNA,cor.prostate.Affy, rownames(ttest.prostate.cDNA) [1:100],

rownames(ttest.prostate.Affy) $[1: 100], 0.5)$

pogr.array["ttest",i,1,j]<- pogr(cor.prostate.cDNA,cor.prostate.Affy, rownames(ttest.prostate.cDNA)[1:50], 
rownames(ttest.prostate.Affy) $[1: 50], 0.5)$

\#\# Need to also go other direction

pogr.array2["ttest",i,4,j] <- pogr(cor.prostate.Affy,cor.prostate.cDNA, rownames(ttest.prostate.Affy) $[1: 1000]$,

rownames(ttest.prostate.cDNA) $[1: 1000], 0.5)$

pogr.array2["ttest",i,3,j] <- pogr(cor.prostate.Affy,cor.prostate.cDNA, rownames(ttest.prostate.Affy) [1:500],

rownames(ttest.prostate.cDNA)[1:500], 0.5)

pogr.array2["ttest",i,2,j] <- pogr(cor.prostate.Affy,cor.prostate.cDNA, rownames(ttest.prostate.Affy) [1:100],

rownames(ttest.prostate.cDNA)[1:100], 0.5)

pogr.array2["ttest",i,1,j] <- pogr(cor.prostate.Affy,cor.prostate.cDNA, rownames(ttest.prostate.Affy) [1:50],

rownames(ttest.prostate.cDNA) $[1: 50], 0.5$ )

npogr.array["ttest",i,4,j] <- (pogr.array["ttest",i,4,j] - EPOGR[i,4])/(1-EPOGR[i,4])

npogr.array["ttest",i,3,j] <- (pogr.array["ttest",i,3,j] - EPOGR[i,3])/(1-EPOGR[i,3])

npogr.array["ttest",i,2,j] <- (pogr.array["ttest",i,2,j] - EPOGR[i,2])/(1-EPOGR[i,2])

npogr.array["ttest",i, 1,j] <- (pogr.array["ttest",i,1,j] - EPOGR[i,1])/(1-EPOGR[i,1])

npogr.array2["ttest",i,4,j] <- (pogr.array2["ttest",i,4,j] - EPOGR[i,4])/(1-EPOGR[i,4])

npogr.array2["ttest",i,3,j] <- (pogr.array2["ttest",i,3,j] - EPOGR[i,3])/(1-EPOGR $[\mathrm{i}, 3])$

npogr.array2["ttest",i,2,j] <- (pogr.array2["ttest",i,2,j] - EPOGR[i,2])/(1-EPOGR[i,2])

npogr.array $2[" t t e s t ", i, 1, j]<-($ pogr.array $[" t t e s t ", i, 1, j]$ - EPOGR[i,1])/(1-EPOGR[i,1])

\#\# cutoff $=0.7$

pogr.array. $0.7[$ "ttest",i,4,j] <- pogr(cor.prostate.cDNA,cor.prostate.Affy, rownames(ttest.prostate.cDNA)[1:1000],

rownames(ttest.prostate.Affy) $[1: 1000], 0.65$ )

pogr.array.0.7["ttest",i,3,j] <- pogr(cor.prostate.cDNA,cor.prostate.Affy, rownames(ttest.prostate.cDNA)[1:500],

rownames(ttest.prostate.Affy) $[1: 500], 0.65$ )

pogr.array. $0.7[$ "ttest",i,2,j] <- pogr(cor.prostate.cDNA,cor.prostate.Affy, rownames(ttest.prostate.cDNA) [1:100],

rownames(ttest.prostate.Affy) [1:100], 0.65)

pogr.array. $0.7[" t t e s t ", i, 1, j]<-$ pogr(cor.prostate.cDNA,cor.prostate.Affy, rownames(ttest.prostate.cDNA)[1:50],

rownames(ttest.prostate.Affy) $[1: 50], 0.65$ )

pogr.array2.0.7["ttest",i,4,j] <- pogr(cor.prostate.Affy,cor.prostate.cDNA, rownames(ttest.prostate.Affy) [1:1000],

rownames(ttest.prostate.cDNA)[1:1000], 0.65)

pogr.array2.0.7["ttest",i,3,j] <- pogr(cor.prostate.Affy,cor.prostate.cDNA, rownames(ttest.prostate.Affy) [1:500],

rownames(ttest.prostate.cDNA)[1:500], 0.65)

pogr.array2.0.7["ttest",i,2,j] <- pogr(cor.prostate.Affy,cor.prostate.cDNA, rownames(ttest.prostate.Affy) [1:100],

rownames(ttest.prostate.cDNA)[1:100], 0.65) 
pogr.array2.0.7["ttest",i,1,j] <- pogr(cor.prostate.Affy,cor.prostate.cDNA, rownames(ttest.prostate.Affy) [1:50],

rownames(ttest.prostate.cDNA)[1:50], 0.65)

npogr.array.0.7["ttest",i,4,j] <- (pogr.array.0.7["ttest",i,4,j] - EPOGR[i,4])/(1-EPOGR[i,4]) npogr.array.0.7["ttest",i,3,j] <- (pogr.array.0.7["ttest",i,3,j] - EPOGR[i,3])/(1-EPOGR[i,3]) npogr.array.0.7["ttest",i,2,j] <- (pogr.array.0.7["ttest",i,2,j] - EPOGR[i,2])/(1-EPOGR[i,2]) npogr.array.0.7["ttest",i,1,j] <- (pogr.array.0.7["ttest",i, 1,j] - EPOGR[i,1])/(1-EPOGR[i,1])

npogr.array2.0.7["ttest",i,4,j] <- (pogr.array2.0.7["ttest",i,4,j] - EPOGR[i,4])/(1-EPOGR[i,4]) npogr.array2.0.7["ttest",i,3,j] <- (pogr.array2.0.7["ttest",i,3,j] - EPOGR[i,3])/(1-EPOGR[i,3]) npogr.array2.0.7["ttest",i,2,j] <- (pogr.array2.0.7["ttest",i,2,j] - EPOGR[i,2])/(1-EPOGR[i,2]) npogr.array2.0.7["ttest",i,1,j] <- (pogr.array2.0.7["ttest",i,1,j] - EPOGR[i,1])/(1-EPOGR[i,1]

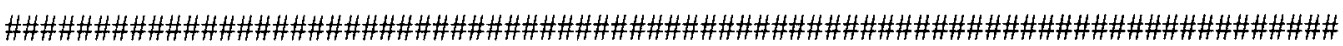
\#\# 2B. E-BAYES (LIMMA) METHOD

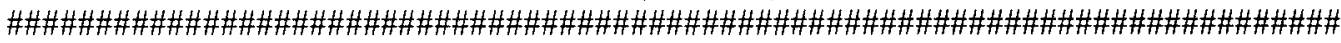

\#\# prostate.cDNA

fit $1<-\operatorname{lm}$ Fit(prostate.cDNA.sub, design 1)

fitcl $<$ - contrasts. fit(fit 1, contr 1)

fitcl $<-$ eBayes(fitcl)

ebayes.prostate.cDNA <- topTable(fitc1, adjust.method="BH",number=1000)

\#\# prostate.Affy

fit2 $<-\operatorname{lm}$ Fit(prostate.Affy.sub, design2)

fitc $2<$ - contrasts.fit(fit 2 , contr2)

fitc2 $<-$ eBayes(fitc2)

ebayes. prostate.Affy <- topTable(fitc2, adjust.method="BH",number=1000)

\#\# First, simple POG score

pog.array["ebayes",i,4,j] <- length(intersect(ebayes.prostate.cDNA $\$ I D[1: 1000]$, ebayes.prostate.Affy $\$$ ID $[1: 1000])) / 1000$

pog.array["ebayes",i,3,j] <- length(intersect(ebayes.prostate.cDNA\$ID[1:500], ebayes.prostate.Affy\$ID[1:500]))/500

pog.array["ebayes",i,2,j] <- length(intersect(ebayes.prostate.cDNA \$ID[1:100], ebayes.prostate.Affy\$ID[1:100]))/100

pog.array["ebayes",i,1,j] <- length(intersect(ebayes.prostate.cDNA\$ID[1:50], ebayes.prostate.Affy\$ID[1:50]))/50

\#\# Normalized POG

npog.array["ebayes",i,4,j] <- (pog.array["ebayes",i,4,j] - EPOG[i,4])/(1-EPOG[i,4])

npog.array["ebayes",i,3,j] <- (pog.array["ebayes",i,3,j] - EPOG[i,3])/(1-EPOG[i,3])

npog.array["ebayes",i,2,j] <- (pog.array["ebayes",i,2,j] - EPOG[i,2])/(1-EPOG[i,2])

npog.array["ebayes",i,1,j] <- (pog.array["ebayes",i,1,j] - EPOG[i,1])/(1-EPOG[i,1])

\#\# Now, look at POGR score

$\#$ \# cutoff $=0.5$

pogr.array["ebayes",i,4,j] <- pogr(cor.prostate.cDNA,cor.prostate.Affy, ebayes.prostate.cDNA $\$ I D[1: 1000]$, ebayes.prostate.Affy $\$ I D[1: 1000]$,

$0.5)$

pogr.array["ebayes",i,3,j] <- pogr(cor.prostate.cDNA,cor.prostate.Affy, ebayes.prostate.cDNA $\$ I D[1: 500]$, ebayes.prostate.Affy $\$ \operatorname{ID}[1: 500], 0.5)$

pogr.array["ebayes",i,2,j]<- pogr(cor.prostate.cDNA,cor.prostate.Affy, 
ebayes.prostate.cDNA $\$ I D[1: 100]$, ebayes.prostate.Affy $\$ I D[1: 100], 0.5)$

pogr.array["ebayes",i,1,j] <- pogr(cor.prostate.cDNA,cor.prostate.Affy, ebayes.prostate.cDNA\$ID[1:50], ebayes.prostate.Affy\$ID[1:50], 0.5)

pogr.array2["ebayes",i,4,j] <- pogr(cor.prostate.Affy,cor.prostate.cDNA, ebayes.prostate.Affy $\$ \operatorname{ID}[1: 1000]$, ebayes.prostate.cDNA $\$ I D[1: 1000]$,

$0.5)$

pogr.array $2[$ "ebayes",i,3,j] <- pogr(cor.prostate.Affy,cor.prostate.cDNA, ebayes.prostate.Affy\$ID[1:500], ebayes.prostate.cDNA\$ID[1:500], 0.5 )

pogr.array2["ebayes",i,2,j] <- pogr(cor.prostate.Affy,cor.prostate.cDNA, ebayes.prostate.Affy $\$ I D[1: 100]$, ebayes.prostate.cDNA $\$ I D[1: 100], \quad 0.5)$

pogr.array $2[$ "ebayes",i,1,j] <- pogr(cor.prostate.Affy,cor.prostate.cDNA, ebayes.prostate.Affy $\$ \operatorname{ID}[1: 50]$, ebayes.prostate.cDNA\$ID[1:50], 0.5 )

npogr.array["ebayes",i,4,j] <- (pogr.array["ebayes",i,4,j] - EPOGR[i,4])/(1-EPOGR[i,4])

npogr.array["ebayes",i,3,j] <- (pogr.array["ebayes",i,3,j] - EPOGR $[\mathrm{i}, 3]) /(1-E P O G R[i, 3])$

npogr.array["ebayes",i,2,j] <- (pogr.array["ebayes",i,2,j] - EPOGR[i,2])/(1-EPOGR[i,2])

npogr.array["ebayes",i,1,j] <- (pogr.array["ebayes",i,1,j] - EPOGR[i,1])/(1-EPOGR[i,1])

npogr.array2["ebayes",i,4,j] <- (pogr.array2["ebayes",i,4,j] - EPOGR[i,4])/(1-EPOGR[i,4])

npogr.array2["ebayes",i,3,j] <- (pogr.array2["ebayes",i,3,j] - EPOGR[i,3])/(1-EPOGR[i,3])

npogr.array2["ebayes",i,2,j] <- (pogr.array2["ebayes",i,2,j] - EPOGR[i,2])/(1-EPOGR[i,2])

npogr.array2["ebayes",i, $1, \mathrm{j}]<-($ pogr.array2["ebayes",i, $1, j]$ - EPOGR[i,1])/(1-EPOGR[i,1])

\#\# cutoff $=0.7$

pogr.array. $0.7[$ "ebayes",i,4,j] <- pogr(cor.prostate.cDNA,cor.prostate.Affy, ebayes.prostate.cDNA $\$ I D[1: 1000]$, ebayes.prostate.Affy $\$ I D[1: 1000]$,

pogr.array.0.7["ebayes",i,3,j] <- pogr(cor.prostate.cDNA,cor.prostate.Affy, ebayes.prostate.cDNA $\$ I D[1: 500]$, ebayes.prostate.Affy $\$ \operatorname{ID}[1: 500], 0.65)$

pogr.array. $0.7[$ "ebayes",i,2,j] <- pogr(cor.prostate.cDNA,cor.prostate.Affy, ebayes.prostate.cDNA\$ID[1:100], ebayes.prostate.Affy\$ID[1:100], 0.65)

pogr.array. $0.7[$ "ebayes",i, $1, j]$ <- pogr(cor.prostate.cDNA,cor.prostate.Affy, ebayes.prostate.cDNA\$ID[1:50], ebayes.prostate.Affy $\$ \operatorname{ID}[1: 50], 0.65)$

pogr.array2.0.7["ebayes",i,4,j]<- pogr(cor.prostate.Affy,cor.prostate.cDNA, ebayes.prostate.Affy $\$ I D[1: 1000]$, ebayes.prostate.cDNA $\$ I D[1: 1000]$,

pogr.array2.0.7["ebayes",i,3,j] <- pogr(cor.prostate.Affy,cor.prostate.cDNA, ebayes.prostate.Affy\$ID[1:500], ebayes.prostate.cDNA\$ID[1:500],

$0.65)$

pogr.array2.0.7["ebayes",i,2,j]<- pogr(cor.prostate.Affy,cor.prostate.cDNA,

$0.65)$ ebayes.prostate.Affy\$ID[1:100], ebayes.prostate.cDNA \$ID[1:100],

pogr.array2.0.7["ebayes",i,1,j]<- pogr(cor.prostate.Affy,cor.prostate.cDNA, ebayes.prostate.Affy $\$ I D[1: 50]$, ebayes.prostate.cDNA $\$ I D[1: 50], 0.65$ )

npogr.array.0.7["ebayes",i,4,j] <- (pogr.array.0.7["ebayes",i,4,j] EPOGR.0.7[i,4])/(1-EPOGR.0.7[i,4])

npogr.array.0.7["ebayes",i,3,j] <- (pogr.array.0.7["ebayes",i,3,j] EPOGR.0.7[i,3])/(1-EPOGR.0.7[i,3])

npogr.array.0.7["ebayes",i,2,j] <- (pogr.array.0.7["ebayes",i,2,j] EPOGR.0.7[i,2])/(1-EPOGR.0.7[i,2])

npogr.array.0.7["ebayes",i,1,j] <- (pogr.array.0.7["ebayes",i, $1, \mathrm{j}]$ - 
EPOGR.0.7[i,1])/(1-EPOGR.0.7[i,1])

npogr.array2.0.7["ebayes",i,4,j] <- (pogr.array2.0.7["ebayes",i,4,j] EPOGR.0.7[i,4])/(1-EPOGR.0.7[i,4])

npogr.array2.0.7["ebayes",i,3,j] <- (pogr.array2.0.7["ebayes",i,3,j] EPOGR.0.7[i,3])/(1-EPOGR.0.7[i,3])

npogr.array2.0.7["ebayes",i,2,j] <- (pogr.array2.0.7["ebayes",i,2,j] EPOGR.0.7[i,2])/(1-EPOGR.0.7[i,2])

npogr.array2.0.7["ebayes",i,1,j] <- (pogr.array2.0.7["ebayes",i, $1, j]$ -

EPOGR.0.7[i,1])/(1-EPOGR.0.7[i,1])

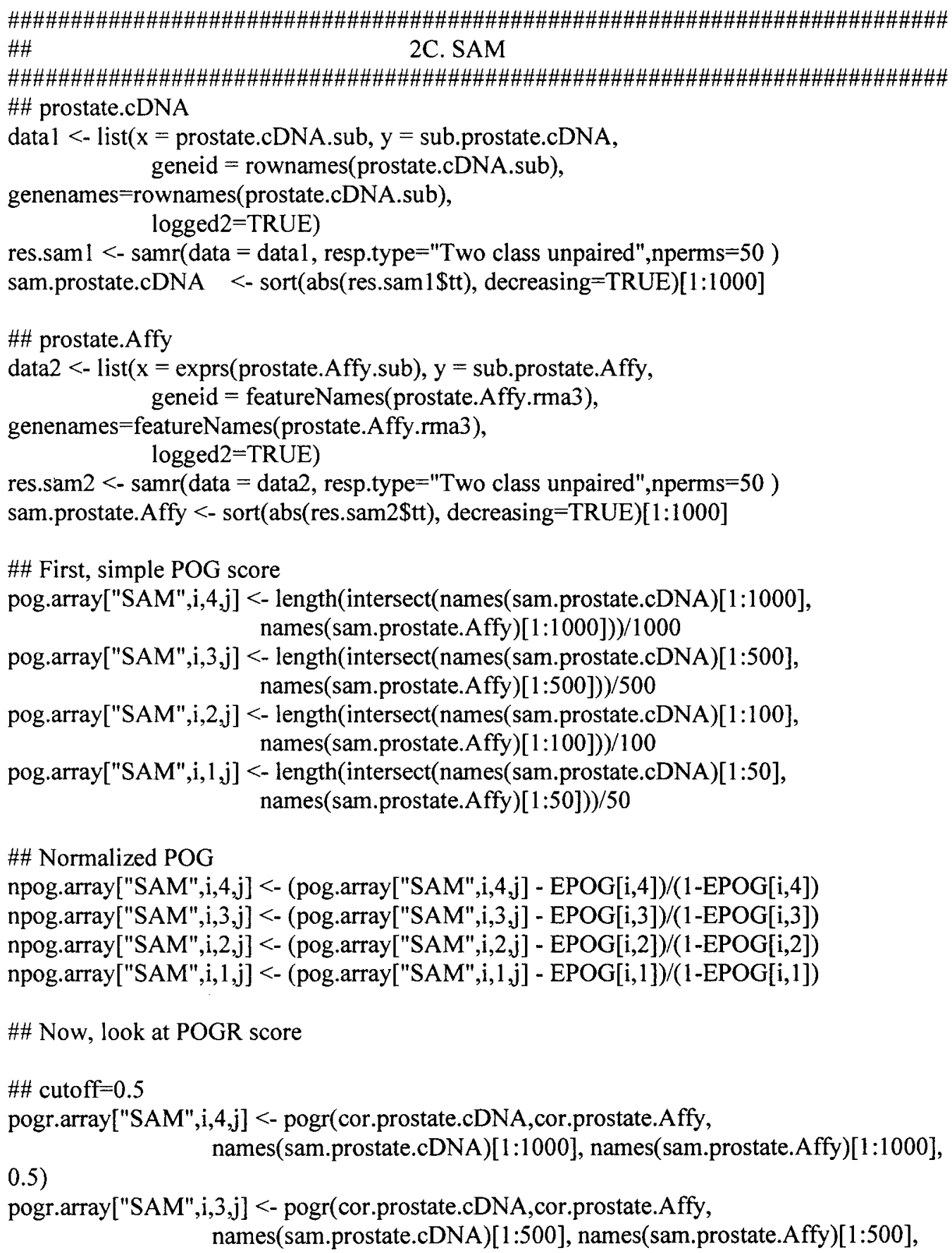


$0.5)$

pogr.array["SAM",i,2,j] <- pogr(cor.prostate.cDNA,cor.prostate.Affy, names(sam.prostate.cDNA)[1:100], names(sam.prostate.Affy)[1:100],

$0.5)$

pogr.array["SAM",i,1,j] <- pogr(cor.prostate.cDNA,cor.prostate.Affy, names(sam.prostate.cDNA)[1:50], names(sam.prostate.Affy)[1:50], 0.5)

pogr.array2["SAM",i,4,j] <- pogr(cor.prostate.Affy,cor.prostate.cDNA, names(sam.prostate.Affy) [1:1000],names(sam.prostate.cDNA)[1:1000],

$0.5)$

pogr.array2["SAM",i,3,j] <- pogr(cor.prostate.Affy,cor.prostate.cDNA, names(sam.prostate.Affy)[1:500],names(sam.prostate.cDNA)[1:500],

$0.5)$

pogr.array2["SAM",i,2,j] <- pogr(cor.prostate.Affy,cor.prostate.cDNA,

$0.5)$ names(sam.prostate.Affy)[1:100],names(sam.prostate.cDNA)[1:100],

pogr.array $2[$ "SAM",i,1,j] <- pogr(cor.prostate.Affy,cor.prostate.cDNA, names(sam.prostate.Affy) $[1: 50]$,names(sam.prostate.cDNA)[1:50], 0.5)

npogr.array["SAM",i,4,j] <- (pogr.array["SAM",i,4,j] - EPOGR[i,4])/(1-EPOGR[i,4])

npogr.array["SAM",i,3,j] <- (pogr.array["SAM",i,3,j] - EPOGR[i,3])/(1-EPOGR[i,3])

npogr.array["SAM",i,2,j] <- (pogr.array["SAM",i,2,j] - EPOGR[i,2])/(1-EPOGR[i,2])

npogr.array["SAM",i,1,j] <- (pogr.array["SAM",i,1,j] - EPOGR[i,1])/(1-EPOGR[i,1])

npogr.array2["SAM",i,4,j] <- (pogr.array2["SAM",i,4,j] - EPOGR[i,4])/(1-EPOGR[i,4])

npogr.array2["SAM",i,3,j] <- (pogr.array2["SAM",i,3,j] - EPOGR[i,3])/(1-EPOGR[i,3])

npogr.array2["SAM",i,2,j] <- (pogr.array2["SAM",i,2,j] - EPOGR[i,2])/(1-EPOGR[i,2])

npogr.array2["SAM",i,1,j] <- (pogr.array2["SAM",i,1,j] - EPOGR[i,1])/(1-EPOGR[i,1])

\#\# cutoff $=0.7$

pogr.array.0.7["SAM",i,4,j] <- pogr(cor.prostate.cDNA,cor.prostate.Affy, names(sam.prostate.cDNA)[1:1000], names(sam.prostate.Affy)[1:1000],

$0.65)$

pogr.array.0.7["SAM",i,3,j] <- pogr(cor.prostate.cDNA,cor.prostate.Affy, names(sam.prostate.cDNA)[1:500], names(sam.prostate.Affy) [1:500],

$0.65)$

pogr.array.0.7["SAM",i,2,j] <- pogr(cor.prostate.cDNA,cor.prostate.Affy, names(sam.prostate.cDNA)[1:100], names(sam.prostate.Affy)[1:100],

pogr.array.0.7["SAM",i,1,j] <- pogr(cor.prostate.cDNA,cor.prostate.Affy, names(sam.prostate.cDNA)[1:50], names(sam.prostate.Affy)[1:50], 0.65)

pogr.array2.0.7["SAM",i,4,j] <-pogr(cor.prostate.Affy,cor.prostate.cDNA, names(sam.prostate.Affy)[1:1000],names(sam.prostate.cDNA)[1:1000],

$0.65)$

pogr.array2.0.7["SAM",i,3,j] <-pogr(cor.prostate.Affy,cor.prostate.cDNA, names(sam.prostate.Affy)[1:500],names(sam.prostate.cDNA)[1:500],

pogr.array2.0.7["SAM",i,2,j] <-pogr(cor.prostate.Affy,cor.prostate.cDNA, names(sam.prostate.Affy) [1:100],names(sam.prostate.cDNA)[1:100],

$0.65)$

pogr.array2.0.7["SAM",i, 1,j] <-pogr(cor.prostate.Affy,cor.prostate.cDNA, names(sam.prostate.Affy)[1:50],names(sam.prostate.cDNA)[1:50],

$0.65)$ 


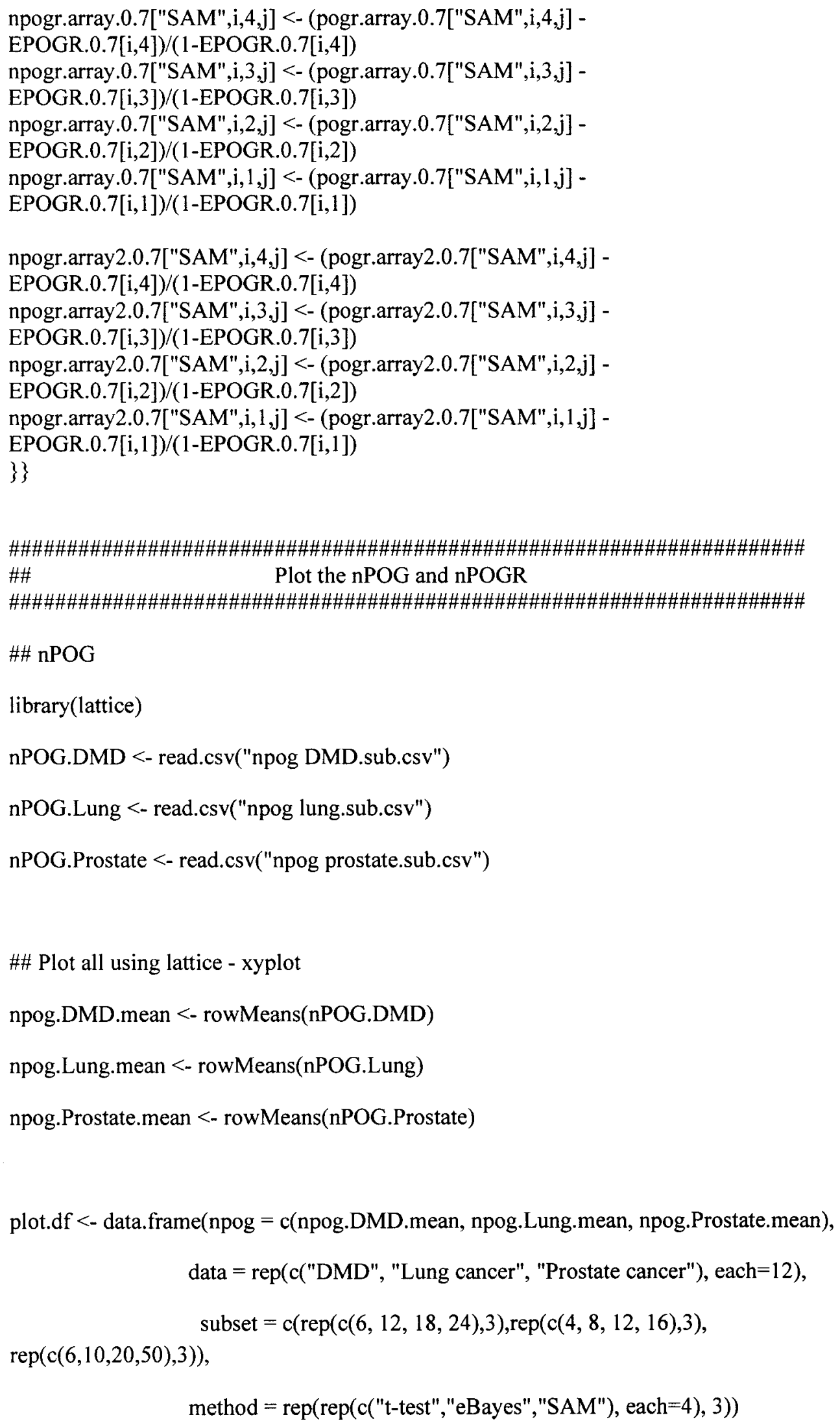


xyplot(npog $\sim$ subset $\mid$ data, groups $=$ method, type $=" 1 "$, data=plot.df, auto.key=list $($ columns $=3$, title $=$ "nPOG score for detecting DEG lists from different datasets of the same diseases" ), layout $=c(3,1)$, scales $=\operatorname{list}(\mathrm{x}=\operatorname{list}(\mathrm{at}=\mathrm{c}(4,8,12,18,24,50))))$

\section{\#\# nPOGR}

nPOGR.DMD <- read.csv("npogr DMD.sub.csv")

nPOGR.Lung <- read.csv("npogr lung.sub.csv")

nPOGR.Prostate <- read.csv("npogr prostate.sub.csv")

\#\# Plot all using lattice - xyplot

npogr.DMD.mean <- rowMeans(nPOGR.DMD)

npogr.Lung.mean <- rowMeans(nPOGR.Lung)

npogr.Prostate.mean <- rowMeans(nPOGR.Prostate)

plot.df $<$ - data.frame(npogr $=\mathrm{c}($ npogr.DMD.mean, npogr.Lung.mean, npogr.Prostate.mean $)$, data = rep(c("DMD", "Lung cancer", "Prostate cancer"), each=12), subset $=c(\operatorname{rep}(c(6,12,18,24), 3), \operatorname{rep}(c(4,8,12,16), 3)$,

$\operatorname{rep}(c(6,10,20,50), 3))$

$$
\text { method = rep(rep(c("t-test", "eBayes", "SAM"), each=4), 3)) }
$$

xyplot(npogr $\sim$ subset $\mid$ data, groups = method, type $=" 1 "$, data=plot.df, auto.key=list $($ columns $=3)$, layout $=\mathrm{c}(3,1)$, scales $=\operatorname{list}(\mathrm{x}=\operatorname{list}(\mathrm{at}=\mathrm{c}(4,8,12,18,24,50))))$

\#\# nPOGR2

nPOGR2.DMD <- read.csv("npogr2 DMD.sub.csv") 
nPOGR2.Lung <- read.csv("npogr2 lung.sub.csv")

nPOGR2.Prostate <- read.csv("npogr2 prostate.sub.csv")

\#\# Plot all using lattice - xyplot

npogr2.DMD.mean <- rowMeans(nPOGR2.DMD)

npogr2.Lung.mean <- rowMeans(nPOGR2.Lung)

npogr2.Prostate.mean <- rowMeans(nPOGR2.Prostate)

plot.df $<$ - data.frame $($ npogr2 $=$ c(npogr2.DMD.mean, npogr2.Lung.mean, npogr2.Prostate.mean),

$$
\begin{aligned}
& \text { data = rep(c("DMD", "Lung cancer", "Prostate cancer"), each=12), } \\
& \text { subset = c(rep(c(6,12,18,24),3),rep(c(4,8,12,16),3), } \\
& \text { 3)), } \\
& \text { method = rep(rep(c("t-test", "eBayes", "SAM"), each=4), 3)) }
\end{aligned}
$$

$\operatorname{rep}(c(6,10,20,50), 3))$,

xyplot(npogr2 subset $\mid$ data, groups $=$ method, type $=" 1 "$, data=plot.df, auto. key $=$ list $($ columns $=3)$, layout $=c(3,1)$, scales $=\operatorname{list}(x=\operatorname{list}(a t=c(4,8,12,18,24,50))))$

\#\# nPOGR 0.75

nPOGR.DMD.0.75<- read.csv("npogr 0.75 DMD.sub.csv")

nPOGR.Lung.0.75<- read.csv("npogr 0.75 lung.sub.csv")

nPOGR.Prostate. $0.75<-$ read.csv("npogr 0.7 prostate.sub.csv")

\#\# Plot all using lattice - xyplot

npogr.DMD.0.75.mean <- rowMeans(nPOGR.DMD.0.75)

npogr.Lung.0.75.mean <- rowMeans(nPOGR.Lung.0.75)

npogr.Prostate.0.75.mean <- rowMeans(nPOGR.Prostate.0.75) 
plot.df <- data.frame(npogr.0.75 = c(npogr.DMD.0.75.mean, npogr.Lung.0.75.mean, npogr.Prostate. 0.75 .mean),

$$
\begin{aligned}
& \text { data }=\operatorname{rep}(c(" D M D ", " L u n g \text { cancer", "Prostate cancer"), each=12), } \\
& \text { subset = } c(\operatorname{rep}(c(6,12,18,24), 3), \operatorname{rep}(c(4,8,12,16), 3), \\
& \text { 3)), } \\
& \text { method = rep(rep(c("t-test", "eBayes", "SAM"), each=4), 3)) }
\end{aligned}
$$

$\operatorname{rep}(c(6,10,20,50), 3))$,

xyplot(npogr.0.75 subset $\mid$ data, groups = method, type="l", data=plot.df, auto.key=list(columns $=3$, title="nPOGR score form listl to list 2 (cutoff= $0.75) ")$, layout $=c(3,1)$,

scales $=\operatorname{list}(x=\operatorname{list}(a t=c(4,8,12,18,24,50))))$

\#\# nPOGR2 0.75

nPOGR2.DMD.0.75<- read.csv("npogr2 0.75 DMD.sub.csv")

nPOGR2.Lung.0.75<- read.csv("npogr2 0.75 lung.sub.csv")

nPOGR2.Prostate. $0.75<-$ read.csv("npogr2 0.7 prostate.sub.csv")

\#\# Plot all using lattice - xyplot

npogr2.DMD.0.75.mean <- rowMeans(nPOGR2.DMD.0.75)

npogr2.Lung.0.75.mean <- rowMeans(nPOGR2.Lung.0.75)

npogr2.Prostate.0.75.mean <- rowMeans(nPOGR2.Prostate.0.75)

plot.df $<$ - data.frame(npogr2.0.75 $=c$ (npogr2.DMD.0.75.mean, npogr2.Lung.0.75.mean, npogr2.Prostate.0.75.mean),

$$
\text { data = rep(c("DMD", "Lung cancer", "Prostate cancer"), each=12), }
$$

subset $=c(\operatorname{rep}(c(6,12,18,24), 3), \operatorname{rep}(c(4,8,12,16), 3)$,

$\operatorname{rep}(c(6,10,20,50), 3))$,

$$
\text { method = rep(rep(c("t-test", "eBayes", "SAM"), each=4), 3)) }
$$


xyplot(npogr2.0.75 subset $\mid$ data, groups = method, type="l", data=plot.df, auto.key=list(columns $=3$,title="nPOGR score form list 2 to list 1 (cutoff= $0.75)$ "), layout $=c(3,1)$, scales $=\operatorname{list}(x=\operatorname{list}(a t=c(4,8,12,18,24,50))))$

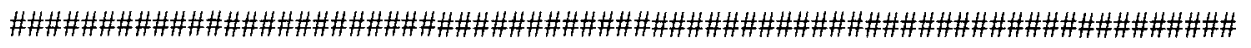
\#\# The End

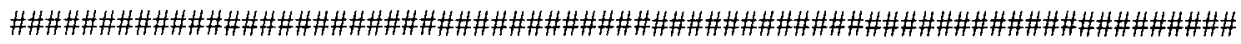

\#\# Codes for Lung Cancer and DMD studies are similar to the Prostate Cancer data sets. 


\section{CURRICULUM VITAE}

Dake Yang

E-mail: D0yang03@cardmail.louisville.edu

Address: 725 E Madison Street Louisville, KY, 40202

EDUCATION

2009.08 - Current MS (candidate, expected in May 2011),

Biostatistics

University of Louisville (UofL), Louisville, Kentucky, USA

2003.09-2007.06 BS, Mathematical Statistics

Beijing Institute of Technology (BIT), Beijing, China

\section{RESEARCH EXPERIENCE}

2010.08--- Now Graduate Student (Guy N. Brock)

Graduate Thesis - Consistency of Differentially Expressed

Gene Rankings Based on Subsets of Microarray Data

Dept of Bioinformatics and Biostatistics,

School of Public Health and Information Sciences, UofL

2009.08-2010.7 Data Analyst and Bioinformatics (Eugenia Wang)

Bioinformatics Data Analysis

Gheens Center on Aging,

School of Medicine, UofL 